University of Tennessee Health Science Center UTHSC Digital Commons

$5-2005$

\title{
First Premolar Extraction Decisions and Effects
}

Ryan Gregory Bowles

University of Tennessee Health Science Center

Follow this and additional works at: https://dc.uthsc.edu/dissertations

Part of the Orthodontics and Orthodontology Commons

\section{Recommended Citation}

Bowles, Ryan Gregory, "First Premolar Extraction Decisions and Effects" (2005). Theses and Dissertations (ETD). Paper 30. http://dx.doi.org/10.21007/etd.cghs.2005.0034.

This Thesis is brought to you for free and open access by the College of Graduate Health Sciences at UTHSC Digital Commons. It has been accepted for inclusion in Theses and Dissertations (ETD) by an authorized administrator of UTHSC Digital Commons. For more information, please contact jwelch30@uthsc.edu. 


\title{
First Premolar Extraction Decisions and Effects
}

\begin{abstract}
The most common method of resolving substantial tooth size-arch length discrepancies (TSALD) is to treat patients with the extraction of premolars. Not all of the extraction space, however, is needed to eliminate the patient's TSALD. Also, it is typical that some of the extraction space will be lost because the molars are used as anchorage units to retract the anterior teeth. OBJECTIVE: This study developed a method to measure changes in the positions of the canines and second premolars relative to the palatal rugae. These data were used to assess how the extraction space was used in resolving the malocclusion. Data were also collected on the severity of the malocclusion-namely, pretreatment overjet, overbite, incisor irregularity, and TSALD-to test whether these were predictive in the extraction decision. The goal was to better understand first premolar extraction changes and decisions. METHODS: Pretreatment and posttreatment dental casts were available for 50 adolescent North American whites with Class I malocclusion who were treated with first-premolar extractions. Sixty-four landmarks were recorded in all three planes of space with a MicroScribe G2X 3D digitizing system and distances were computergenerated using trigonometric algorithms. RESULTS: Removing a first premolar gave an average of 7.3 $\mathrm{mm}$ of space in each quadrant of which $3.8 \mathrm{~mm}(51 \%)$ was consumed by canine retraction, while $3.6 \mathrm{~mm}$ $(49 \%)$ was used as the second premolar moved mesially. Changes in the transverse dimension after premolar extraction treatment were minor, though second premolar width diminished about $1 \mathrm{~mm}$ as these teeth were moved into a narrower segment of the arch $(P<0.01)$. The extent of incisor irregularity had a significant positive association with the amount of canine retraction $(r=0.33 ; P=0.02)$.

CONCLUSION: A four first premolar extraction pattern in Class I patients provides space that is consumed almost equally by the retraction of canines and the mesial movement of the buccal segments in the maxilla. Multivariately, the two significant variables associated with increased canine retraction in these patients were transverse canine change (i.e., buccally displaced canines) and a high level of incisor irregularity.
\end{abstract}

\section{Document Type}

Thesis

Degree Name

Master of Dental Science (MDS)

Program

Orthodontics

Research Advisor

Edward Harris, Ph.D.

\section{Keywords}

First Premolar Extraction, Palatal Rugae, Canine Retraction, Extraction Decisions

\section{Subject Categories}

Dentistry | Medicine and Health Sciences | Orthodontics and Orthodontology 


\title{
FIRST PREMOLAR EXTRACTION DECISIONS AND EFFECTS
}

\author{
A Thesis \\ Presented for \\ The Graduate Studies Council \\ The University of Tennessee \\ Health Science Center
}

\section{In Partial Fulfillment \\ Of the Requirements for the Degree \\ Master of Dental Science \\ From The University of Tennessee}

By

Ryan Gregory Bowles, D.D.S.

May 2005 
Copyright $@$ Ryan Gregory Bowles, 2005 All rights reserved 


\section{DEDICATION}

This thesis is dedicated to my parents

Dr. Richard Matteson Bowles

and

Mrs. Sharon White Bowles

in recognition of their love, guidance and support

of my education 


\section{ACKNOWLEDGMENTS}

I would like to thank my thesis committee for their help and support over these last three years. Dr. Greg Hutchins and Dr. Chuck Robinson contributed with helpful comments and suggestions and Dr. Edward Harris, my committee chairman and research advisor, guided the overall project. I also deeply appreciate the care and concern of my lovely wife, Glenda, as I persevered on this project and increased her own responsibilities in the process. Thank you for standing with me and being a true helper. Finally, I also thank my two wonderful children Amber and Daniel for accepting less attention whenever I worked on this thesis. Don't worry kids, daddy's research days are over! 


\begin{abstract}
The most common method of resolving substantial tooth size-arch length discrepancies (TSALD) is to treat patients with the extraction of premolars. Not all of the extraction space, however, is needed to eliminate the patient's TSALD. Also, it is typical that some of the extraction space will be lost because the molars are used as anchorage units to retract the anterior teeth. OBJECTIVE: This study developed a method to measure changes in the positions of the canines and second premolars relative to the palatal rugae. These data were used to assess how the extraction space was used in resolving the malocclusion. Data were also collected on the severity of the malocclusion - namely, pretreatment overjet, overbite, incisor irregularity, and TSALD - to test whether these were predictive in the extraction decision. The goal was to better understand first premolar extraction changes and decisions. METHODS: Pretreatment and posttreatment dental casts were available for 50 adolescent North American whites with Class I malocclusion who were treated with first-premolar extractions. Sixty-four landmarks were recorded in all three planes of space with a MicroScribe G2X 3D digitizing system and distances were computer-generated using trigonometric algorithms. RESULTS: Removing a first premolar gave an average of $7.3 \mathrm{~mm}$ of space in each quadrant of which $3.8 \mathrm{~mm}(51 \%)$ was consumed by canine retraction, while $3.6 \mathrm{~mm}(49 \%)$ was used as the second premolar moved mesially. Changes in the transverse dimension after premolar extraction treatment were
\end{abstract}


minor, though second premolar width diminished about $1 \mathrm{~mm}$ as these teeth were moved into a narrower segment of the arch $(\mathrm{P}<0.01)$. The extent of incisor irregularity had a significant positive association with the amount of canine retraction $(\mathrm{r}=0.33 ; \mathrm{P}=0.02)$. CONCLUSION: A four first premolar extraction pattern in Class I patients provides space that is consumed almost equally by the retraction of canines and the mesial movement of the buccal segments in the maxilla. Multivariately, the two significant variables associated with increased canine retraction in these patients were transverse canine change (i.e., buccally displaced canines) and a high level of incisor irregularity. 


\section{TABLE OF CONTENTS}

$\begin{array}{lll}\text { Chapter } & \text { Page }\end{array}$

I. INTRODUCTION .................................................... 1

II. REVIEW OF THE LITERATURE................................. 3

Quantifying Extraction Decisions.............................. 3

First Premolar Extractions............................... 4

Second Premolar Extractions.............................. 5

Other Considerations................................... 7

Premolar Extraction Effects................................... 8

Incisor Angulation................................... 9

Incisor versus Molar Changes........................ 11

Incisor Retraction............................. 11

Molar Protraction............................... 15

Occlusal Plane........................................... 17

Vertical Dimension.................................... 18

Arch Changes........................................ 18

Arch Width........................................ 19

Arch Depth.................................... 21

Tooth Size-Arch Length Discrepancy................... 21

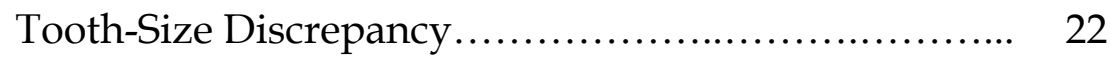

Stability of Palatal Rugae...................................... 23 
Summary and Conclusions................................. 28

III. MATERIALS AND METHODS ..................................... 29

Materials.................................................. 29

Sample Characteristics................................ 29

Data Analysis............................................ 30

Methods......................................................... 31

Cast Analysis.......................................... 31

Statistical Analysis..................................... 37

IV. RESULTS ......................................................... 39

Side Differences............................................ 39

Sexual Dimorphism......................................... 43

Extraction Space........................................... 47

Use of the Extraction Space................................. 54

Multivariate Model............................................. 60

V. DISCUSSION …................................................. 63

Side Differences............................................. 64

Sexual Dimorphism............................................. 65

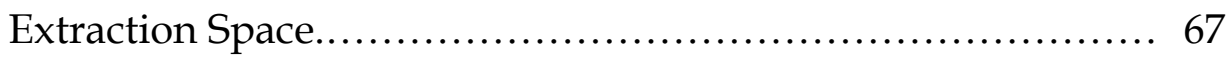

Mesiodistal Dimension.................................. 67

Transverse Dimension................................. 69 
Predictive Models............................................. $\quad 70$

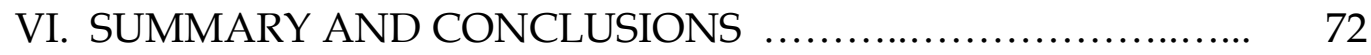

LIST OF REFERENCES....................................... $\quad 76$

APPENDIX F................................................ 84

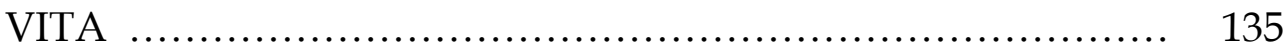




\section{LIST OF TABLES}

Table

1 Summary of the average amounts of maxillary incisor retraction as measured in four studies with five different references

2 Results of paired t-tests assessing side differences in the mesiodistal plane...

3 Results of paired t-tests assessing side differences in the transverse plane.

4 Results of one-way ANOVA testing for sexual dimorphism in dental movements

5 Results of one-way ANOVA testing for sexual dimorphism in dental relationships

6 Descriptive statistics for changes in tooth positions

$7 \quad$ Results of linear regression predicting the amount of canine retraction.

8 Results of stepwise linear regression predicting the amount of canine retraction... 


\section{LIST OF FIGURES}

Figure

Page

1 Demonstration of the effect of occlusal plane tipping on the molar's relationship to the medial ruga point

2 Pretreatment cast landmarks (Points 1-34)............................. 32

3 Posttreatment cast landmarks (Points 37-66)..................... 33

4 Sequenced array of cases according to the amount of observed mesiodistal change in the distal contact of the maxillary canine.

5 Sequenced array of cases according to the amount of observed mesiodistal change in the mesial contact of the maxillary second premolar.

6 Sequenced array of cases according to the amount of observed transverse change in the distal contact of the maxillary canine

7 Sequenced array of cases according to the amount of observed transverse change in the mesial contact of the maxillary second premolar.

8 The association between the amount of canine retraction and the degree of maxillary incisor irregularity at pretreatment.

9 The association between the amount of canine retraction and the amount of transverse change in canine position 


\section{CHAPTER I}

\section{INTRODUCTION}

A common orthodontic problem occurs when the tooth crown dimensions exceed the available space in the alveolar arch. This discrepancy occurs when the sum of the mesiodistal crown diameters exceed the bony arch perimeter available for the teeth to be properly aligned. As methods are unavailable to increase arch size by promoting bone growth, the common alternative is to reduce tooth size.

By far, the most common method of resolving substantial tooth size-arch length discrepancies (TSALD) is to treat patients with the extraction of premolars. Extracting a premolar can create more space than is needed to resolve the discrepancy. Premolar extraction is a quantum event in that removing a tooth provides about $7 \mathrm{~mm}$ of space whereas the alternative, nonextraction, provides none. It is unlikely, however, that all of the extraction space (ca. $15 \mathrm{~mm}$ per arch) is needed to eliminate the patient's TSALD. Additionally, it is almost certain that some of the extraction space will be lost because the molars are used as anchorage units to retract the anterior teeth distally. The molar units provide more anchorage than the anterior teeth because of their multiple, large roots, but they are not immobile when pitted against the anterior teeth. Anchorage will be 
lost, so some of the extraction space will be consumed by the posterior teeth moving mesially during retraction of the anterior teeth (Williams and Hosila 1976). In fact, this mesial molar movement is so easily controlled that orthodontists often use the extraction of second premolars to adjust the buccal segment relationship (de Castro 1974). This extraction pattern can be beneficial in the mandible to correct Class II malocclusions into a Class I buccal segment relationship and also in the maxilla to correct Class III malocclusions (Schoppe 1964). Slippage of molar anchorage commonly is intentionally encouraged to close the unneeded portion of the extraction space.

The purpose of the present research is to answer some questions that arise when the treatment plan includes first premolar extractions. In particular, this paper analyzes how much of the extraction space is typically used to resolve problems such as anterior, mid-arch and posterior tooth crowding (TSALD) and overjet and how much is used as the buccal segments move mesially to close the rest of the extraction space. Also, an examination of pretreatment factors is used to allow us to quantify when first premolar extraction decisions are made. That is, are there measurable pretreatment conditions, such as the extent of incisor irregularity and degree of overbite that might be predictive of the amount of first premolar space used? Collectively, these two areas of inquiry are related as they provide guidelines that contribute to a better understanding of first premolar extraction changes and decisions. 


\section{CHAPTER II}

\section{REVIEW OF THE LITERATURE}

This Review of the Literature of premolar extraction decisions and effects consists of three broad topics, (1) quantifying extraction decisions, (2) premolar extraction effects and (3) stability of the palatal rugae. This chapter presents a discussion of each of these topics in turn.

\section{Quantifying Extraction Decisions}

While the frequency of premolar extraction cases varies among orthodontists, it is estimated that one-third of all orthodontic patients have such a severe malocclusion that some pattern of premolar extraction is deemed necessary in order to resolve the problems and align the teeth (Proffit 2000:24956). TSALD is the most important factor necessitating the decision to extract premolars (Baumrind et al. 1996). Differential diagnosis involves determining whether first or second premolars should be extracted in the maxilla and/or in the mandible. Basic guidelines towards choosing premolar extraction patterns are available in textbooks (e.g., Moyers 1973; Proffit 2000), but more detailed factors for consideration are available in the primary orthodontic literature. 


\section{First Premolar Extractions}

General guidelines suggest extracting first premolars when the TSALD source area is primarily in the anterior portion of the arch. Removing the first premolars is a straightforward way to correct anterior crowding, excessive overjet and protrusion. This correction works by making space for the alignment and retraction of incisors and canines. Extracting premolars close to the area of crowding is beneficial because at the point when crowding or protrusion is corrected, little extraction space remains to be closed (Schoppe 1964; Graber 1972; Dewel 1973; Moyers 1973; Proffit 2000).

Other indications for a first premolar extraction pattern instead of a second premolar extraction pattern include excessive overbite, Class II, division 2 malocclusion type, and serial extraction therapy. Brandt and Safirstein (1975) stated that placing the extraction site closer to the anterior gives a mechanical advantage in leveling the arch as space is closed. This advantage is helpful when treating patients with a deep bite. Creekmore (1997) said that, as a rule of thumb, he preferred extracting maxillary first premolars for non-surgical treatments of non-growing Class II, division 2 malocclusions. He specified that in these cases he treated the mandible without premolar extraction. Dewel (1973) specified another reason for first premolar extractions over second premolar extractions. He stated that when treating a young patient with serial extraction, who will 
require the removal of permanent teeth, the first premolars should be removed so that erupting canines can more easily drift distally into the extraction space.

\section{Second Premolar Extractions}

The basic indication for second premolar extraction is that there is moderate anterior crowding with no protrusion and the patient has good facial balance (Dewel 1955, 1973; Graber 1972; Moyers 1973; Brandt and Safirstein 1975; Creekmore 1997; Proffit 2000). The crowding guideline is subjective as de Castro (1974) describes a second premolar extraction instance as being when there is a TSALD of $5 \mathrm{~mm}$ or more, while Schoppe (1964) describes it as being a TSALD of $7.5 \mathrm{~mm}$ or less. Either way, removing the second premolars will give enough space to resolve minor crowding while not changing the profile. It also leaves the incisors in their original position over basal bone without inclining them lingually which is undesirable (Dewel 1955; Schoppe 1964).

Other considerations for removing second premolars instead of first premolars include posterior crowding, anterior open bite, Class III correction, and facilitation of intentional anchorage slippage. When second or third molars are crowded, ectopic, or impacted, they can be helped by creating space in the buccal segments of the arch. This space is created by extracting second premolars so that the first molar can move mesially (Logan 1973; de Castro 1974). This extraction pattern is also advantageous for correcting anterior open bites, 
because it is easier to accentuate the curve of Spee and lessen relapse of the open bite after treatment (Brandt and Safirstein 1975). Also, by reducing the posterior vertical dimension through the removal of posterior occlusal surface area, an immediate increase in anterior overbite is accomplished, facilitating closure of an open bite (Logan 1973). Second premolar extraction in the maxilla can also be helpful in camouflaging Class III malocclusions when combined with a first premolar extraction in the mandible. This pattern allows more retraction of the lower incisors while allowing more mesial maxillary molar movement to correct the malocclusion (Schoppe 1964). Intentional anchorage slippage can be facilitated by the extraction of second premolars. This is desirable when there will be excess extraction space remaining after TSALD resolution and the patient has good facial harmony. De Castro (1974) specifies that when needing to move the molars forward more than $2.5 \mathrm{~mm}$ on each side, a second premolar extraction pattern is indicated. By removing second premolars instead of first premolars, first molars are easily slipped forward instead of necessitating unwanted retraction of the anterior teeth to close the remaining space (Dewel 1955; Schoppe 1964; Dewel 1973; Logan 1973; de Castro 1974). This occurs not only because the teeth mesial to the first molars are removed, giving a clear path of movement, but also because the weaker anterior anchorage is increased from six to eight teeth producing more resistance (Dewel 1973). 


\section{Other Considerations}

Individual tooth conditions should also be considered before deciding which premolars to extract. When teeth are carious, ankylosed, or impacted, special consideration should be given to extracting these teeth instead of other healthy teeth. Also, when teeth are congenitally missing, this can affect extraction pattern, because an equivalent tooth should be extracted to maintain arch symmetry (Brandt and Safirstein 1975; Proffit 2000).

Arch symmetry is an important factor is choosing tooth extraction patterns. If asymmetrical extraction or tooth loss occurs within an arch, the midline will shift to the side of the arch with the extraction space closest to the anterior. Therefore, when premolars are extracted, they are typically extracted in left-right pairs in order to maintain this arch symmetry (Proffit 2000:211).

While arch position is a consideration in choosing which premolars to extract, size differences between first and second premolars are insignificant. The average size difference between first and second premolars was not mentioned in any of the reviewed articles as an issue in determining extraction patterns. 


\section{$\underline{\text { Premolar Extraction Effects }}$}

Much has been written over the years about changes that occur with premolar extractions. Premolar extraction treatment is well described because of its widespread usage. Keim et al. (2002) reported from a survey of 789 orthodontists that over $95 \%$ had extracted teeth in the last year. Virtually every orthodontist extracts premolars in some situations as it helps greatly with crowding, protrusion, retraction, vertical problems, and other factors (Proffit 2000). The only meaningful debate on extraction today centers not on whether it should be done, but rather in what malocclusions it should be done and which teeth should be extracted.

However, history shows that this was not always the trend. At the end of the nineteenth century, Angle staunchly opposed all extraction therapy because he contended every person had the potential for the ideal relationship of all his teeth. This proposition led to his use of expansion appliances and elastics to achieve correct overbite and overjet and make room for the teeth. An article of faith for Angle was that a correct occlusion would result in maximum esthetics and stability. He wrote that if the final placement of the teeth was not stable, it simply pointed to the orthodontist's failure in not putting the teeth in an ideal occlusion, not to any failure in his theory (Proffit 2000:250). In the 1920s, Case debated Angle's student Dewey over this issue and proposed the need for extraction treatments, but nonextraction sentiment prevailed through the late 
1930s. By the 1940s, the observed propensity for relapse in nonextraction treatment caused many clinicians to reconsider the extraction question. Tweed (1944:406) stated that:

I also maintain that when a discrepancy between tooth pattern and basal bone does exist, it is far better to remove dental units to bring about a balance between tooth anatomy and basal bone; and that if this correction is made, our patients will be benefited by a nearer approach to the normal than is possible if we retain all the dental units and in so doing are compelled to displace all the teeth off the dental ridge and into protrusion.

Others, like Begg (1977), also helped promote a strong reemergence of extraction treatments through the mid twentieth century (Proffit 2000). In recent times, claims that related temporomandibular dysfunction to the extraction of upper first premolars decreased extraction rate, but today premolar extractions are commonly used as adjuncts to treatment (Proffit 2000).

\section{Incisor Angulation}

With the increased space available in the arch following the extraction of a pair of premolars, the orthodontist can use some or all of that space to correct anterior tooth inclinations and relationships. Bishara, Cummins and Zaher (1997) contrasted groups of Class II, division I patients treated by extraction and nonextraction. They concluded that treatment that includes first premolar extraction results in a significantly more obtuse U1 to L1 interincisal angle than 
nonextraction treatments. However, no descriptions were given of the amount of crowding, overjet, or overbite with which the patients initially presented.

Carter (1988) showed that the interincisal angle increased an average of 22 degrees in a group of first premolar extraction patients treated with edgewise appliances. There was no account given of starting conditions for these patients. Bishara et al. (1995) found in a study of Class II patients treated with first premolar extraction, that the increase in this angle was 4.3 degrees in males and 12.3 degrees in females. Conversely, there was a 4.6 degree decrease in males and a 1.1 degree decrease in females treated without extractions. This increased amount of angulation seen in extraction treatments is not due to changes occurring in only one arch. It is the result of changes that occur to both the upper and lower incisors as they are retracted several millimeters and uprighted (Darendeliler and Taner-Sarisoy 2001).

Ong and Woods (2001) conducted a cephalometric and cast study comparing first and second premolar extraction patterns in the maxilla. Relating the maxillary incisors to the A-Pogonion reference line, they showed that a significant reduction in incisor protrusion and proclination was noted regardless of which maxillary premolar was extracted. However, the extraction pattern was relevant as there was a maxillary incisor angulation reduction of 8.2 degrees in the first premolar extraction group compared to an angulation reduction of 3.3 degrees in the second premolar extraction group. No difference in the data 
between males and females was reported. Because the malocclusions that were selected for the first or second premolar extraction pattern were of different types and severity these data are not directly comparable.

\section{Incisor versus Molar Changes}

Using a compressive force between the mesial and distal segments to close premolar extraction space will affect teeth in both regions to different degrees. This difference occurs because large multi-rooted teeth resist forces more than single-rooted anterior teeth with ovoid cross-sections (Jepsen 1963). The result is that molars have more of a bodily movement, while the incisor crowns tend to move more than their roots as a combination of bodily movement and tipping. Also, the clinician's preferences and goals in treatment often dictate mechanics that enhance these biological effects. This combination results in more anterior retraction than would occur with bodily movement alone (Williams and Hosila 1976).

Incisor Retraction

If the extraction area is closer to the anterior region, a greater amount of incisor retraction can occur. This statement has been borne out by several researchers to varying degrees (Williams and Hosila 1976; Steyn, du Preez and Harris 1997; Ong and Woods 2001). 
First premolar extraction treatments have been measured to give an average $4.7 \mathrm{~mm}$ of incisor retraction in the maxilla in relation to the facial plane (Nasion-Pogonion), while second premolar extractions yielded a similar $4.2 \mathrm{~mm}$ mean incisor retraction (Steyn, du Preez and Harris 1997). Ong and Woods (2001) studied treatment groups containing a mixture of Class I and II patients. They found more significant differences with a maxillary incisor retraction of $4.2 \mathrm{~mm}$ related to the A-Pogonion line in the first premolar extraction group and a 2.3 $\mathrm{mm}$ incisor retraction in the second premolar extraction group. Using the palatal anatomy as a reference point and the Begg technique for treatment, Williams and Hosila (1976) found combined upper and lower retraction to be $10.3 \mathrm{~mm}$ in a four first premolar extraction group. They concluded that when taking out first premolars, " ... approximately 66.5 per cent of the available extraction space was occupied by retracted anterior segments." This agrees with Creekmore's (1997) rule of thumb that you use two thirds of first premolar extraction space for incisor retraction and for the correction of crowding, and the other one third is used by the buccal segments.

Extraction patterns involving a combination of maxillary first premolars and mandibular second premolars are too variable in the resultant amount of incisor retraction to make valuable conclusions. A mean of $9.3 \mathrm{~mm}$ of total retraction was found in an upper first and lower second premolar extraction group by Williams and Hosila (1976). Ong and Woods (2001) recorded $3.7 \mathrm{~mm}$ 
of maxillary incisor retraction with this extraction pattern, while Steyn, du Preez and Harris (1997) recorded $6.6 \mathrm{~mm}$ of maxillary incisor retraction.

Incisor retraction can be greatly affected by the use of headgear, because it reduces the amount of anchorage that is lost during retraction of the incisors and preserves more of the extraction space for retraction. Schwab (1963) states that the use of headgear can increase the attainable amount of incisor retraction in second premolar cases up to the level of first premolar extraction cases without headgear. In the previously mentioned research on incisor retraction, none of the treatment groups used headgear, but there are several more studies reporting incisor retraction in which headgear use is a factor. Table 1 summarizes the findings of these studies. Cusimano, McLaughlin and Zernik (1993) studied a group of Class I or II high-angle patients with four first premolars extracted. Using the contour of the palate as a reference, they found there was an average $1.9 \mathrm{~mm}$ of maxillary incisor retraction. Carter (1988), using the Nasion-Pogonion reference line to the maxillary incisal edge, recorded a mean of $8.2 \mathrm{~mm}$ of incisor retraction in Class II first premolar extraction cases. Measuring to the incisor apex, he recorded only $0.6 \mathrm{~mm}$ of retraction. Bishara et al. (1995) showed a mean retraction of $4.6 \mathrm{~mm}$ in the maxillary anterior in Class II first premolar extraction cases using A-Pogonion as a reference. The Nasion-A line was used as a reference by Luppanapornlarp and Johnston (1993). They found a mean of 2.8 
Table 1. Summary of the average amounts of maxillary incisor retraction as measured in four studies with five different references.

\begin{tabular}{ll}
\hline \multicolumn{1}{c}{ Reference } & Mean \\
\hline Palatal Contour & $1.9 \mathrm{~mm}$ \\
Nasion-Pogonion to Incisal Edge & $8.2 \mathrm{~mm}$ \\
Nasion-Pogonion to Incisor Apex & $0.6 \mathrm{~mm}$ \\
A-Pogonion & $4.6 \mathrm{~mm}$ \\
Nasion-A & $2.8 \mathrm{~mm}$ \\
\hline
\end{tabular}


$\mathrm{mm}$ of maxillary incisor retraction in a group of Class II patients treated with first premolar extractions.

The limitation of using these studies to evaluate the use of extraction space is that the samples cannot be comparable at the start of treatment. The extraction patterns used were not randomized, but based on what changes the clinicians were intending to develop. Thus, the differential treatments should lead to different amounts of incisor retraction if the orthodontists are competent.

\section{$\underline{\text { Molar Protraction }}$}

In contrast to retraction that occurs in the anterior segment is a mesial movement that occurs in the posterior segment with the molars. Less study has been done on the movement of these teeth than the incisors, but some data have been collected. Like the incisor retraction studies, the relevancy of these studies is confounded because the patient groups had different pretreatment characteristics which led to their different extraction patterns. In premolar extraction groups without the use of headgear, Ong and Woods (2001) compared amounts of molar protraction. The four first premolar extraction group was made up of mostly Class II subjects, while the four second premolar extraction group was almost entirely Class I in molar relationship. They found that the mean forward movement of the maxillary first molar ranged from $3.7 \mathrm{~mm}$ in four first premolar extraction groups to $4.5 \mathrm{~mm}$ in four second premolar extraction 
groups. Comparing this movement to the retraction of incisors in those cases revealed that $80 \%$ of the time, the molars experienced greater movement than the incisors, and, thus, the majority of space created by extraction was filled by mesial movement of the molars. They found that this occurred most frequently in the patients who had increased crowding and no natural spacing between any teeth. Williams and Hosila (1976) recorded that without headgear use, the combined upper and lower posterior protraction was $5.2 \mathrm{~mm}$ in their four first premolar extraction group and $7.2 \mathrm{~mm}$ in their group with upper first and lower second premolar extractions. These results showed more anchorage loss occurred when the extraction sites were more posterior.

Like incisor retraction, headgear may have an effect on the amount of molar protraction recorded as shown by Ashmore et al. (2002). Using palatal rugae, they recorded that the maxillary molar moved an average of $2.2 \mathrm{~mm}$ distally in a Class II treatment group using headgear. They showed that molars move mesially almost a full millimeter over a 24 month period in the absence of treatment. Combining these two findings, an adjusted mean molar distalization of $3.0 \mathrm{~mm}$ is found when headgear is used. This result shows why headgear use must be considered in the analysis, since it can drastically decrease molar protraction.

Other studies using various treatments including headgear include Carter (1988) who, using a perpendicular to Sella-Nasion reference line, found a $3.8 \mathrm{~mm}$ 
maxillary molar protraction in a group treated with first premolar extractions and headgear. Counter-intuitively, he found in another premolar extraction group treated with the Begg appliance and no headgear that the maxillary molar protraction was virtually identical at $3.4 \mathrm{~mm}$. Luppanapornlarp and Johnston (1993) used the Pterygoid Vertical reference line and found a $2.4 \mathrm{~mm}$ molar protraction in patients treated with first premolar extraction. Cusimano, McLaughlin and Zernik (1993) recorded a mean of $5.0 \mathrm{~mm}$ maxillary molar protraction in a high angle Class I or II first premolar extraction group using the palate contour as a reference.

\section{Occlusal Plane}

It is controversial whether a change to the inclination of the occlusal plane occurs secondary to premolar extraction. No difference has been observed clinically although it has been theorized. Sheppe (1969), using plastic teeth arranged in wax arch forms to represent ideal dentitions, predicted occlusal changes from premolar extraction by carrying out the extractions and treatment on the teeth in the wax. He stated there should be a change in the occlusal plane due to retraction of the mandibular anterior region. His prediction showed that, as the incisors are retracted, the crowns move more lingually than the roots, so the teeth become more upright. This uprighting causes Downs' occlusal plane to tip superiorly in the anterior. Cephalometric studies show that this change never 
takes place, at least to any clinically detectable level (Darendelier and TanerSarisoy 2001). This is in agreement with Luppanapornlarp and Johnston (1993) who found that there was no significant change in occlusal plane as related to the Sella-Nasion line in extraction and in nonextraction groups.

\section{Vertical Dimension}

Research on the vertical dimension in premolar extraction situations is limited to cephalometric studies at this time. Staggers (1994) and Kocadereli (1999) investigated what differences occur in the vertical dimension between nonextraction groups and extraction groups. Vertical changes occurring after first premolar extractions were no different from those observed in nonextraction cases. In both groups an increase in the vertical dimension was found. As Staggers notes, "Most of orthodontic mechanics are extrusive in nature, and this extrusion appears to maintain or even increase the vertical dimension." The fact that extrusion occurred equivalently in both groups seems to place doubt on to whether premolar extraction can be linked to TMJ disorders (Staggers 1994).

\section{Arch Changes}

Various changes take place in arch form during orthodontic treatment. Depending on the sort of treatment, there can be an increase or decrease in arch width and arch length. 


\section{$\underline{\text { Arch Width }}$}

Using the standard edgewise appliance, the clinician can expect some expansion of the arch width, because the wire is engaged into bracket slots that are on the labial or buccal sides of the teeth. Orthodontists have suggested that, with premolar extraction treatment, the removal of teeth negates this expansion and leads to a "collapse" of the arch, yielding narrower intercanine, interincisal, and intermolar widths. Research directed on arch width has not agreed with the collapse premise. In several studies, not only has it been shown that there is an increase in arch width in extraction cases, but that there is more arch width expansion when the patient is treated with premolar extractions than when they are not (Bishara et al. 1994; O'Higgins and Lee 2000; Gianelly 2003). How premolar extractions can lead to arch width expansion is hard to explain, but O'Higgins and Lee (2000) and Gianelly (2003) consider that it is probably related to moving the canines distally into a wider arch diameter where the premolars were while maintaining intermolar width. Along these lines, Gianelly (2003) and Bishara et al. (1994) showed that there is a significant increase in intercanine width in extraction cases compared to nonextraction cases, but they differed in the amount of change they reported in intermolar width. However, the relevancy of these findings is diminished since the groups are not comparable in the amount of change needed. Gianelly (2003) used orthodontic models to compare Class I and II patients treated with the extraction of four first premolars 
to Class I, II, and III patients treated with no extractions. He found that there was an overall increase in intercanine width in both groups with mandibular intercanine width in the extraction group being significantly more than the nonextraction group. He found no significant change in the intermolar width between the extraction and nonextraction groups. Bishara et al. (1994) measured casts on Class II patients treated with four first premolar extractions and found a significantly greater increase in maxillary and mandibular intercanine widths in that group when compared to a Class II group treated without extractions. Net intercanine expansion was $3.2 \mathrm{~mm}$ in males and $3.1 \mathrm{~mm}$ in females, compared to only a $0.5 \mathrm{~mm}$ and $2.0 \mathrm{~mm}$ expansion, respectively, in the nonextraction group. They found a net decrease in intermolar width in the extraction group while the nonextraction group had an increase in this dimension. Whether or not arch width expansion remains after treatment is a different matter, and this aspect has been explored by Vaden, Harris and Gardner (1997). They found from cast measurements that although the arches become narrower with age, there still was an overall increase in arch width in premolar extraction cases up to 15 years posttreatment. 


\section{Arch Depth}

An extraction pattern with premolar extractions will result in a mesiodistal tooth structure loss of around $15 \mathrm{~mm}$ per arch. This will result in a decrease of the anteroposterior dimension of the arch depth, but to what extent? All of the studies reviewed concluded that there was a significant reduction in arch depth following premolar extractions (Bishara et al. 1994; Bishara, Cummins and Zaher 1997; Vaden, Harris and Ziegler 1997; O’Higgins and Lee 2000). O'Higgins and Lee (2000) found that arch depth reduces to a greater extent than even the mesiodistal width of the premolars based on orthodontic cast studies. Vaden, Harris and Gardner (1997) stated that the arches progressively lose arch length with age. These results indicate that overjet and anteroposterior changes are not directly coordinated with mesiodistal tooth structure loss, because other factors such as arch shape, width and age must be considered.

\section{Tooth Size-Arch Length Discrepancy}

The difference between tooth structure and arch length available to support the tooth structure is a relevant concern for the orthodontist. It would be beneficial to understand how premolar extractions affect this discrepancy during and after treatment, because treatment decisions are affected by this factor. Bishara et al. (1994) confirmed what was already supposed when they concluded that extracting premolars significantly diminishes the discrepancy 
between tooth size and arch length. Using cast measurements, it was found in another study that there was a gradual increase in TSALD in both extraction and nonextraction treatment groups following the end of treatment as both groups experienced instability (Bishara, Cummins and Zaher 1997). O'Higgins and Lee (2000) theorized from conducting a study using plastic teeth in wax arch forms that the arch length space available after premolar extractions should be greater than even the size of the premolars removed due to the phenomenon of retracting the incisors into a larger arch diameter as discussed above.

\section{Tooth-Size Discrepancy}

Tooth-size discrepancy is a disharmony between mesiodistal widths of the maxillary and mandibular arch as described by Bolton (1958). A study by Saatci and Yukay (1997) on 50 patients with no pretreatment tooth-size discrepancy used Bolton's analysis to determine if the removal of first or second premolars would produce any discrepancy. A significant finding was that in 31 of 50 patients, a tooth-size discrepancy resulted from first premolar extraction treatments. However, the amount of increase in the tooth-size discrepancy was negligible at $0.4 \mathrm{~mm}$. In second premolar extraction treatments, no significant tooth-size discrepancy resulted. It should be noted that this study was not done by comparing pretreatment and posttreatment casts, but rather by simulating 
extractions on the pretreatment casts by using a computer program to compute the resulting discrepancies.

\section{Stability of Palatal Rugae}

An area in which it is important to describe change during premolar extractions is the palate. Its importance comes not in how it affects making treatment decisions, but in the way it may be used as a landmark for dental research. Lysell (1955) developed a classification system for the palatal rugae pairs, and, using a symmetrograph, described their general stability. His study did not include changes that occur during orthodontic treatment, but it did describe the effects that extractions without treatment have on the rugae. He concluded that "extractions have a local but no general effect on the direction of the rugae." The uniqueness and overall stability of the rugae suggested their use for forensics and even general anthropological studies of paternity determination. This appreciation for the rugae as unique and stable landmarks is further substantiated insofar as English et al. (1988) concluded that the palatal rugae pattern is sufficiently characteristic to discriminate between individuals; they found it was legitimate to base identification upon their comparison.

Many studies have shown that rugal landmarks are applicable for dental research specifically. Van der Linden (1978) concluded that little change takes place in rugae length and interruga distance through growth using the occlusal 
plane as a reference. He stated that the rugae points demonstrate "...remarkable stability in their anteroposterior relationships to each other and lend themselves quite well to the analysis of the changes in mesiodistal locations in buccal teeth in normal developing dental arches." This result was based on cast and cephalometric measurements in children from 6 to 16 years old without orthodontic treatment. In another smaller group of treated subjects, he did the same analysis and found that orthodontic treatment can influence rugae position, particularly anteroposterior distances between medial and lateral points of the same rugae. Most of the changes were due to movement in the lateral and anterior rugae points only, so the posterior medial rugae points were advocated as stable landmarks for research involving orthodontic treatment. This finding was similar to Hausser's study (1950) that described rugae movement after extraction of teeth. He observed the lateral parts moved half the distance that the teeth moved, while the medial parts did not seem to move at all (cited in Lysell 1955). The stability of the medial points over the lateral points was also observed by Peavy and Kendrick (1967) who said that, the "closer the rugae are to the teeth, the more prone they are to 'stretch' in the direction that their associated teeth move." Almeida et al. (1995) demonstrated in a cast study that these medial rugae points were stable in both the transverse and sagittal planes and useful for longitudinal cast analysis even when the patients were treated with headgear or functional appliances. 
In addition to these findings of the importance of using medial points, has been the finding that the more posterior rugae are less susceptible to changes with tooth movement. In a study of patients treated with maxillary first premolar extractions it was concluded that rugae in the canine area were unstable moving $1.9 \mathrm{~mm}$ on average, but the most posterior rugae averaged only $0.2 \mathrm{~mm}$ of movement and were stable (Peavy and Kendrick 1967). In another study, orthodontic treatment was shown to change rugae position in the sagittal dimension more in the anterior region (Almeida et al. 1995). There was greater anteroposterior change in medial points between the first and second pair of rugae, going from anterior to posterior, compared to between the second and third pair. The shortcoming of this data is that it cannot be determined which rugae points were moving to cause this change.

Although posterior rugae are more stable in general, it is the third palatal rugae pair in particular that has been shown to be the most stable reference for evaluating transverse and anteroposterior change. Bailey, Esmailnejad and Almeida (1996) studied palatal rugae changes in adults undergoing both extraction and nonextraction treatment and concluded that the third palatal rugae points are best for evaluating tooth movement in a linear, transverse, and anteroposterior direction, regardless of treatment method. Hoggan and Sadowsky (2001) used cephalometry to evaluate the anteroposterior measurements that were derived by using palatal rugae. Their findings also 
suggested the accuracy of using the mesial point of the third pair of rugae. They concluded that measuring sagittal distances by relating the teeth to this landmark was as accurate as measurements from a cephalogram and suggested that to determine sagittal anchorage loss, “... progress dental casts can be used instead of a lateral cephalometric radiograph."

When using palatal rugae points as landmarks, their association with the occlusal plane is important. The occlusal plane is often used as a reference plane to relate the teeth to the rugae, but it often changes during growth and orthodontic treatment. Van der Linden (1978) has shown that a 3 degree decrease in the occlusal plane results in a distal displacement of $1 \mathrm{~mm}$ for a molar point being related to a medial ruga point on the occlusal plane. This change occurs because the perpendicular lines dropped to the occlusal plane are separated as the plane rotates counter-clockwise causing increased distance between the molar and the ruga point. The increased distance makes it appear that the molars have moved distally. This movement masks some of the mesial movement of molars if a correction is not made for the change in occlusal plane (Fig. 1).

In sum, research on rugae suggests the use of medial points of the third (distal) palatal rugae as stable landmarks for determining tooth movement. 


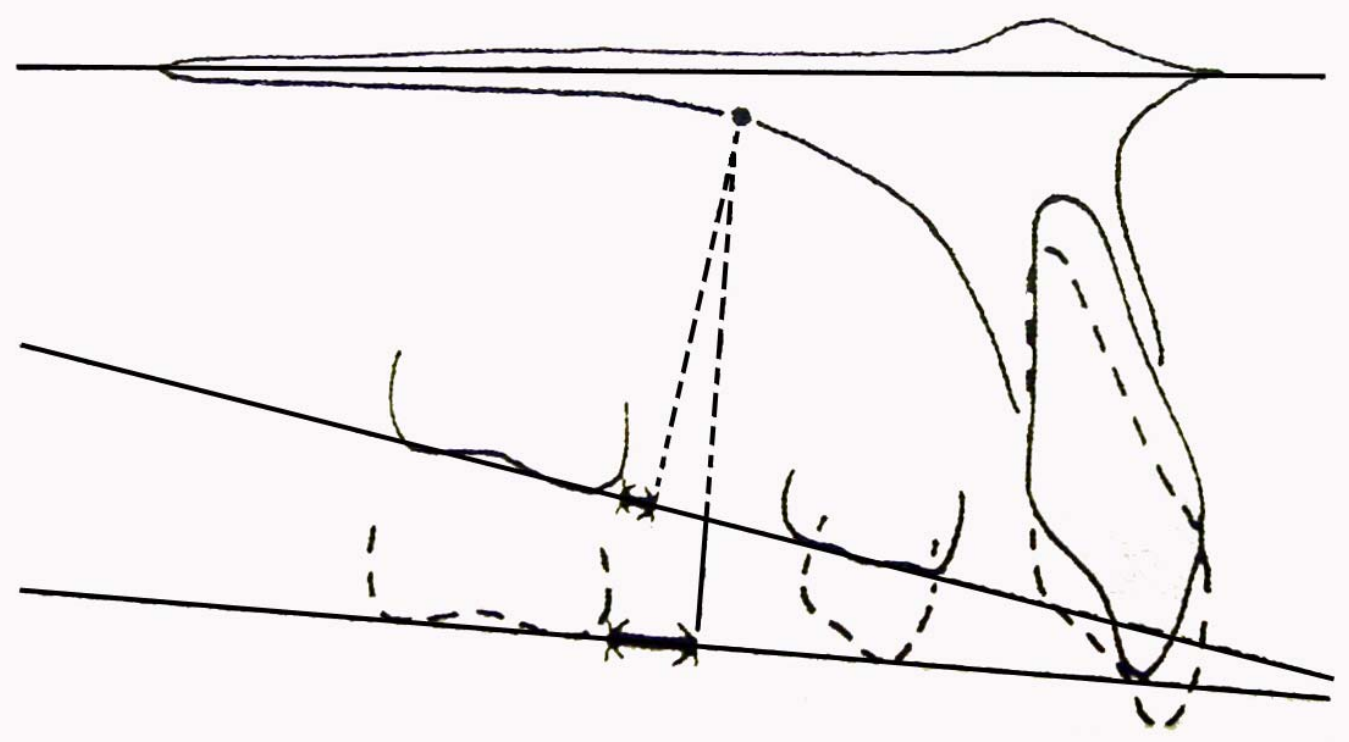

Fig. 1. Demonstration of the effect of occlusal plane tipping on the molar's relationship to the medial ruga point. On the average, a decrease of 3 degrees in the angle will result in a distal displacement of $1 \mathrm{~mm}$ of the tooth in relation to the medial ruga point. 


\section{$\underline{\text { Summary and Conclusions }}$}

The purpose of this section was to review what the literature states concerning differential premolar extraction decisions and effects. Previous research has attempted to provide guidelines for choosing between a first and a second premolar extraction pattern. As the literature suggests, the extraction pattern can have vastly different effects on characteristics of the occlusion. It is important to evaluate these considerations when choosing an extraction pattern, especially how premolar extraction space is used relative to extraction pattern. Thus, a review was given of the changes that result from different extraction patterns including changes that must be accounted for when doing research on tooth movement. Finally, the stability of the palatal rugae was reviewed for its usefulness as a stable landmark for dental research on this topic. 


\section{CHAPTER III}

\section{MATERIALS AND METHODS}

These materials and methods were used to study tooth movements after the extraction of first premolars. In particular, the mesial and distal movement of teeth adjacent to the extraction space was examined.

\section{$\underline{\text { Materials }}$}

\section{Sample Characteristics}

A sample of 50 completed orthodontic cases was collected from the archived records of the graduate orthodontic program at the University of Tennessee, Memphis. This sample size is adequate in order to generate significant data and reduce type II errors according to statistical calculation. The sample group consists of adolescent North American whites who were treated with first premolar extractions. Twenty-nine of the sample patients are female and the remaining twenty-one patients are male. All of these patients had a Class I malocclusion at the start of treatment. Cases were selected at random that met the following three criteria, (1) all permanent teeth were present and fullyerupted, disregarding third molars, (2) each case was maintained in a Class I 
buccal segment relationship, and (3) each case was treated with the extraction of first premolars, one per each quadrant. These delineations were established in order to constrain the sample variability and thus reduce the risk of type II errors.

\section{Data Analysis}

Data analysis for this project can be divided into two sections, a descriptive portion and a hypothesis driven section. The first step was to generate descriptive statistics for the sample according to the extraction pattern of first premolars in all quadrants. These data would illuminate in what way the extraction space is used to resolve the malocclusion. In particular, one goal of the research was to define what amount of the extraction space was used to unravel the anterior teeth and reduce overjet and compare it to the amount of space taken up by buccal segment advancement. These data were collected by measuring changes in the position of the teeth adjacent to the extraction space in reference to the palatal rugae. The changes in position of the canine and second premolar relative to the palatal rugae as a stable reference point show how the space was used in resolving the malocclusion.

In addition to analyzing the descriptive statistics, an important facet of this study is to understand what other features of the pretreatment malocclusion are predictive of how the premolar extraction space is used. To answer this question, the aspects of the pretreatment malocclusion that lead an orthodontist 
to select maxillary first premolar extractions in their treatment plan instead of another extraction pattern must be quantified. Predictive relationships were tested for in this area using incisor overjet, overbite, TSALD, and incisor irregularity relationships as the independent variables and comparing them to the amounts of canine retraction.

\section{$\underline{\text { Methods }}$}

\section{Cast Analysis}

Analysis was limited to changes in the maxilla where the medial endpoints of the third pair of palatal rugae can be used as fiducial landmarks against which tooth movements can be qualified (van der Linden 1978; Almeida et al. 1995; Bailey et al. 1996; Hoggan and Sadowsky 2001). Accuracy of landmark identification was improved by wearing $3 X$ dental loupes. The following sets of landmarks were marked on the pre- and posttreatment casts using a mechanical pencil with $0.5 \mathrm{~mm}$ lead. The same (homologous) landmarks were marked on each pair of casts. These landmarks are illustrated in Figures 2 and 3.

A. The mesial and distal limits of the midpalatal raphe, which are used to define the midline of the dental arch (Points 29-30; 65-6). 


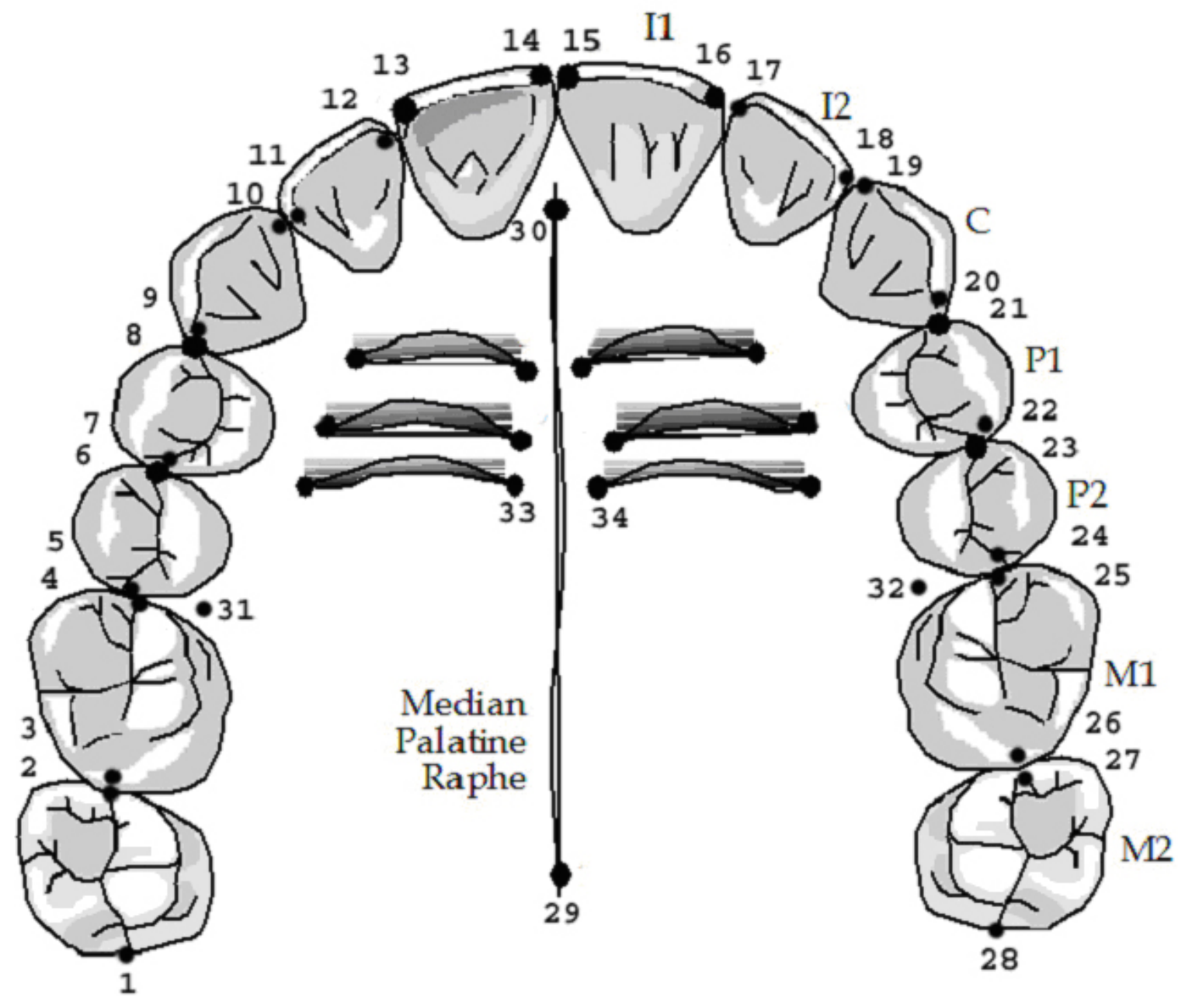

Fig. 2. Pretreatment cast landmarks (Points 1-34). 


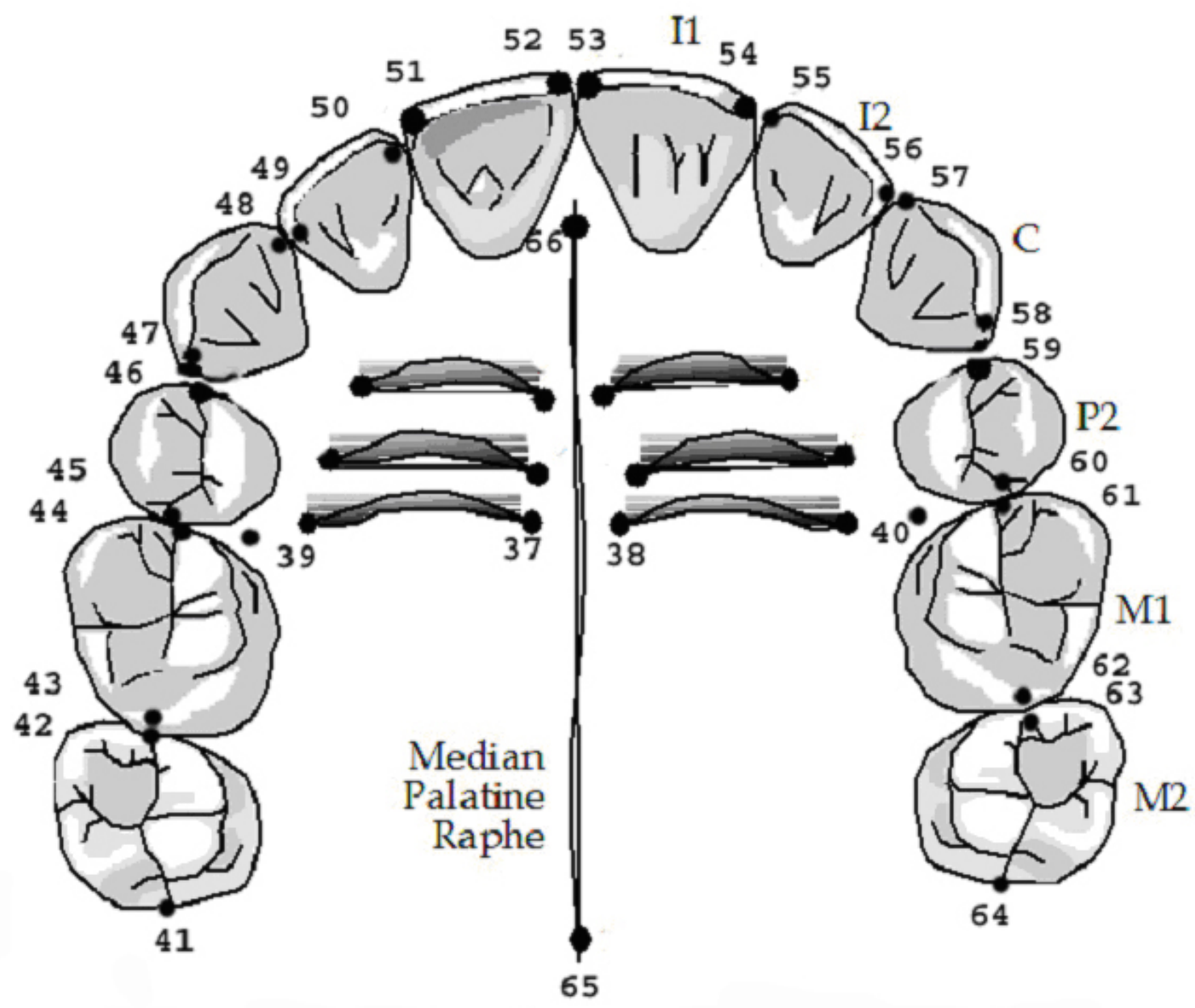

Fig. 3. Posttreatment cast landmarks (Points 37-66). 
B. The distal anatomic contact and the mesial anatomic contacts were marked from second molar to second molar, adjusting for any rotation due to the malocclusion. Because many teeth contact each other along a broad zone of contact rather that at a one point contact, these points were marked in the middle of the anatomical contact zone. As a result, oftentimes these middle contact areas are not coincident even though the teeth are aligned sufficiently to place them in full intercuspation. Points are located independently in the left and right quadrants (Points 1-28 and 41-64).

C. The medial and lateral limits of each of the three major pairs of palatal rugae are marked (Lysell 1955). Based on prior analysis (e.g., Almeida et al. 1995; Bailey et al. 1996), the distal rugae are most stable during orthodontic treatment, so the medial limits of the distal left-right pair of rugae are used as the fiducial points against which tooth movements are measured (Points 3334 and 37-38).

D. Points were also marked in the palatal embrasure area between the first molar and second premolar for use as additional reference positions as needed (Points 31-32 and 39-40).

The sixty-four landmarks marked on the casts were digitized in three dimensions with a MicroScribe G2X 3D digitizing system. This digitizer is comparable to a CAD/CAM instrument and accurate up to 0.009 inches or 0.23 $\mathrm{mm}$. The Cartesian coordinates inputted were then used to machine-generate 
the desired straight-line distances between landmarks using Rhinoceros 2.0 (Robert McNeel \& Associates, Seattle, WA). The occlusal plane, which was defined in this study as including the mesial incisal edge of the most procumbent central incisor and the mesiobuccal cusp tips of the two first molars, was used as a reference line on which we could determine the mesiodistal and mediolateral distances. The midpalatal raphe defined the mesiodistal direction in the plane while the mediolateral direction was perpendicular to it in the plane.

Using the straight-line measurements taken from the digitizer, the mesiodistal, mediolateral, and craniocaudal components of those distances between the rugae and the teeth were computer-generated using trigonometric algorithms. That is, we purposely computed the relationship of the incisor, canine and premolar to the rugae in three individual dimensions and not just the straight-line distances. Acquiring these one-dimensional measurements will allow an omission of any undesired component of change when analyzing the data. For example, since craniocaudal changes do not directly contribute to closing the premolar extraction space it might be helpful to edit those changes out when looking at the data. Results of the pre- and posttreatment analyses were entered into an Excel ${ }^{\circledR}$ spreadsheet and the treatment changes were obtained by subtraction. Errors were minimized by superimposing the pre- and posttreatment casts in Rhinoceros 2.0 and comparing the tooth movement measurements there with the final data in the $\operatorname{Excel}^{\circledR}$ spreadsheets. 
Additionally, the maxillary and mandibular casts were occluded in maximum intercuspation, and incisor overjet and overbite was measured using the method of Baume et al. (1973), using digital-readout sliding calipers. Pretreatment overjet is a measure of the need for incisor retraction, while the treatment change in overjet is one measure of the amount of anterior tooth movement accomplished. The degree of overbite at pretreatment is important in determining whether intrusive or extrusive movement of teeth will be needed during treatment (Proffit 2000:200).

Finally, an analysis of anterior crowding was accomplished by using two methods. First, a modification of the Little's Incisor Irregularity Index was used on the pretreatment casts. This measurement was determined by the method described by Little with the modification being that maxillary casts were measured and not mandibular casts (Little 1975). Also, the Microscribe G2X and Rhinocerus 2.0 were used to measure the discrepancies between the contact points instead of calipers. Second, a modified Nance analysis of anterior crowding was conducted on the maxilla pretreatment casts only (Nance 1947). Total TSALD was calculated with the modified Nance approach by subtracting the sum of the individual tooth sizes of the teeth anterior to the first permanent molars from the total arch length. Individual tooth sizes were measured from mesial contact point to distal contact point using the Microscribe G2X and Rhinocerus 2.0 and then summed to find total space needed. Total arch length 
was measured using digital-readout sliding calipers in four segments consisting of the first molar to canine and canine to the mesial of the central incisor on each side. When the sum of the individual tooth sizes is subtracted from the overall arch length, a negative sum shows crowding and a positive sum reveals spacing.

\section{Statistical Analysis}

The statistical analysis of the resultant data was performed on a computer using StatView 5.0.1 (SAS Institute Inc., Cary, NC). This program was first used to do repeated measures ANOVA tests to distinguish left and right size differences. These differences were examined in the anteroposterior and transverse dimensions at the distal of the canines and the mesial of the second premolars.

Once the left and right side differences had been calculated, descriptive statistics for overall treatment changes were then generated along with one sample t-tests. The average of the right and left sides were used for all values that related to the canine and second premolar landmarks. Descriptive statistics were generated for changes in the anteroposterior dimension and transverse dimension at the distal of the canines and the mesial of the second premolars.

Unpaired t-tests were used to determine if sex differences existed. The variables tested for sexual dimorphism are the following: total change in the anteroposterior and transverse dimensions at the distal of the canines and mesial 
of the second premolars, pretreatment overjet, posttreatment overjet, change in overjet, pretreatment overbite, posttreatment overbite, change in overbite, tooth size arch length discrepancy and incisor irregularity. The right and left sides of the casts were again averaged for the statistical tests of sexual dimorphism that included the canines and second premolars.

Finally, linear regression analysis was used for quantifying the first premolar extraction decisions for this sample and comparing them to characteristics of the pretreatment occlusion. In this analysis, average positional change at the distal of the canines was compared with overjet, overbite, tooth size arch length discrepancy, and incisor irregularity.

All the data were collated and arranged in Microsoft Excel 2000®. Data were graphed using Delta Graph 4.0.5 (Delta Point, Inc., Monterey, CA) to show the anteroposterior and transverse changes at the canines and second premolars, using registration points at the medial tips of the posterior palatal rugae. 


\section{CHAPTER IV}

\section{RESULTS}

This chapter describes the spatial changes of the maxillary teeth in orthodontic cases treated with extraction of four first premolars. The coordinate data collected from pretreatment and posttreatment casts are evaluated statistically in this chapter: One, descriptive statistics for the changes that occur to the maxillary teeth are presented and described. Two, several variables were tested for sexual dimorphism, with the thought that dimorphic traits require a different analysis (that accounts for the sex differences) than if the sexes can be combined. Three, the last section examines predictive relationships between pretreatment characteristics and premolar extraction decisions. The data were derived from pretreatment and posttreatment patients treated with the extraction of four first premolars. Only the maxillary pairs of dental casts were measured in this study.

\section{$\underline{\text { Side Differences }}$}

An initial issue was to test for left-right side asymmetries. Of course, the dental arch is essentially symmetric bilaterally, but there can be minor asymmetries that are either random or systematic (Lu 1966; BeGole 1980). The 
occurrence of directional asymmetries - where one side systematically has a different size or shape than the other (Van Valen 1962; Palmer and Strobeck 2003) - was tested for in the mesiodistal and the transverse (buccolingual) planes. With the coordinate system used in the present study, the $X$ axis is mesiodistal and the $\mathrm{Y}$ axis is transverse. None of the tests for left-right differences was significant for the mesiodistal axis (Table 2). Specifically, the maxillary canine and the second premolar each was equidistant in the dental arch vis-à-vis the palatal rugae (1) at the start of treatment and (2) at the end of treatment and (3) the amounts of tooth movement of these two types were statistically equivalent in the two quadrants. These statistical results indicate that there is front-to-back (mesiodistal) symmetry in these tooth positions. More correctly, the results fail to disclose discernible arch asymmetry for the teeth measured - which is not a forgone conclusion given prior studies that have documented systematic archsize asymmetries in the dentition (e.g., Woo 1931, 1938; Lu 1966; Cassidy 1996). These results also show that, along the mesiodistal axis, orthodontic treatment did not introduce any systematic bias.

Results are more complicated for the transverse axis (Table 3). The buccolingual distances from the rugae point to the canine and to the second premolar in the two quadrants were statistically the same at the start of treatment. The left and the right canine was about $17.4 \mathrm{~mm}$ lateral of its ruga point. And, the second premolars were about $18 \mathrm{~mm}$ from the landmarks. But, 
Table 2. Results of paired $t$-tests assessing side differences in the mesiodistal plane.

\begin{tabular}{lcccccc}
\hline Variable & Left & Right & $\begin{array}{c}\text { Mean } \\
\text { Difference }\end{array}$ & sem & t-test & P-value \\
\hline Pretreatment & & & & & & \\
Canine & 7.07 & 6.66 & 0.41 & 0.3728 & 1.10 & 0.2754 \\
Second Premolar & 1.68 & 1.90 & 0.22 & 0.3466 & 0.63 & 0.5285 \\
Posttreatment & & & & & & \\
Canine & 3.16 & 3.03 & 0.13 & 0.2678 & 0.47 & 0.6389 \\
Second Premolar & 1.90 & 1.64 & 0.26 & 0.2602 & 1.01 & 0.3196 \\
Treatment Change & & & & & & \\
Canine & 3.92 & 3.63 & 0.28 & 0.2905 & 0.98 & 0.3314 \\
Second Premolar & 3.58 & 3.54 & 0.04 & 0.2871 & 0.14 & 0.8854 \\
\hline
\end{tabular}


Table 3. Results of paired t-tests assessing side differences in the transverse plane.

\begin{tabular}{|c|c|c|c|c|c|c|}
\hline Variable & Left & Right & $\begin{array}{c}\text { Mean } \\
\text { Difference }\end{array}$ & sem & t-test & P-value \\
\hline \multicolumn{7}{|l|}{ Pretreatment } \\
\hline Canine & 17.36 & 17.39 & 0.03 & 0.3205 & 0.09 & 0.9309 \\
\hline Second Premolar & 17.85 & 18.21 & 0.36 & 0.3183 & 1.14 & 0.2588 \\
\hline \multicolumn{7}{|l|}{ Posttreatment } \\
\hline Canine & 18.07 & 16.47 & 1.60 & 0.3032 & 5.29 & $<0.0001$ \\
\hline Second Premolar & 16.71 & 15.14 & 1.57 & 0.2982 & 5.27 & $<0.0001$ \\
\hline \multicolumn{7}{|l|}{ Treatment Change } \\
\hline Canine & 0.71 & -0.92 & 1.63 & 0.3037 & 5.37 & $<0.0001$ \\
\hline Second Premolar & 1.13 & -3.07 & 4.20 & 0.3894 & 10.80 & $<0.0001$ \\
\hline
\end{tabular}


during treatment, the left canine experienced expansion vis-à-vis the ruga, an average of $0.7 \mathrm{~mm}$, whereas the right canine was moved medially an average of $0.9 \mathrm{~mm}$. Both of these mean in-treatment changes were highly significant statistically by one-sample t-tests. Importantly, these different treatment effects in the two quadrants caused the posttreatment position of the canines to be asymmetric $(\mathrm{P}<0.0001)$, with the left canine farther to the buccal than the right canine. However, this difference between sides only averages $1.6 \mathrm{~mm}$, so it is not visually obvious. Prior studies - that have measured intercanine width rather than quadrant-specific changes - could not have detected this difference.

The same pattern of changes occurred for the second premolar, though the asymmetry was greater. There was no side difference at the start of treatment (Table 3), both widths averaging about $18 \mathrm{~mm}$. During treatment, the left premolar was moved laterally about $1 \mathrm{~mm}$ while the right premolar was moved medially an average of $3 \mathrm{~mm}$. These side differences created a highly significant left-right asymmetry at the end of treatment $(\mathrm{P}<0.0001)$. Again, the unique measurement protocol used in this study likely accounts for the novel detection of these asymmetries.

\section{$\underline{\text { Sexual Dimorphism }}$}

The variables measured in the present study primarily relate tooth positions (specifically their contact points) to the palatal rugae; the 
measurements are not "size" variables per se. It is not surprising, then, that there is little evidence of sexual dimorphism (Table 4). Instead, this section is provided primarily for completeness.

Two of the anteroposterior variables achieved marginal statistical significance, namely the distal contact of $\mathrm{U} 3$ at the end of treatment $(\mathrm{P}=0.03)$ and the mesial contact of U5 at the end of treatment $(\mathrm{P}=0.06)$. Inspection of the means shows that these two contact points are farther from the rugae landmarks in males than females. This is concordant with the well-known observation that males have appreciably larger palates than females, both anteroposteriorly and transversely (e.g., Knott 1961; Sillman 1964), and the additional spacing among palatodental landmarks seen here is just another facet of the generalized size difference between the sexes.

We also collected data on some potential predictor variables, and tests for sexual dimorphism in these features are shown in Table 5. There was no statistically significant sex difference in the conventional measures of the severity of malocclusion, which agrees with prior epidemiological studies (e.g., Kelly and Harvey 1977). The tooth size-arch size discrepancy of Nance (1947) was computed, and this requires measuring the space required (i.e., the sum of mesiodistal crown dimensions) and space available (i.e., size of the arch perimeter). It is confirmatory of the methods that both of these dimensions are 
Table 4. Results of one-way ANOVA testing for sexual dimorphism in dental movements.

\begin{tabular}{|c|c|c|c|c|c|c|c|c|}
\hline \multirow[b]{2}{*}{ Variable } & \multicolumn{3}{|c|}{ Males } & \multicolumn{3}{|c|}{ Females } & \multirow[b]{2}{*}{ F Ratio } & \multirow[b]{2}{*}{ Prob $>F$} \\
\hline & $\bar{n}$ & Mean & sem & $\bar{n}$ & Mean & sem & & \\
\hline Pretreatment & \multicolumn{6}{|c|}{ Anteroposterior } & & \\
\hline Canine & 21 & -7.25 & 0.55 & 29 & -6.24 & 0.46 & 2.02 & 0.1622 \\
\hline Second Premolar & 21 & 1.53 & 0.56 & 29 & 2.17 & 0.48 & 0.76 & 0.3875 \\
\hline \multicolumn{9}{|l|}{ Posttreatment } \\
\hline Canine & 21 & -3.87 & 0.50 & 29 & -2.43 & 0.43 & 4.76 & 0.0341 \\
\hline Second Premolar & 21 & -2.35 & 0.49 & 29 & -1.13 & 0.42 & 3.58 & 0.0644 \\
\hline \multicolumn{9}{|l|}{ Treatment Change } \\
\hline Canine & 21 & 3.38 & 0.34 & 29 & 3.81 & 0.29 & 0.90 & 0.3488 \\
\hline Second Premolar & 21 & -3.88 & 0.31 & 29 & -3.30 & 0.26 & 2.04 & 0.1595 \\
\hline Pretreatment & \multicolumn{6}{|c|}{ Transverse } & & \\
\hline Canine & 21 & 17.47 & 0.52 & 29 & 17.33 & 0.44 & 0.04 & 0.8412 \\
\hline Second Premolar & 21 & 18.87 & 0.53 & 29 & 17.73 & 0.45 & 2.66 & 0.1096 \\
\hline \multicolumn{9}{|l|}{ Posttreatment } \\
\hline Canine & 21 & 16.68 & 0.29 & 29 & 16.31 & 0.25 & 0.92 & 0.3412 \\
\hline Second Premolar & 21 & 15.53 & 0.35 & 29 & 14.86 & 0.30 & 2.20 & 0.1441 \\
\hline \multicolumn{9}{|l|}{ Treatment Change } \\
\hline Canine & 21 & -0.79 & 0.39 & 29 & -1.02 & 0.33 & 0.21 & 0.6483 \\
\hline Second Premolar & 21 & -3.34 & 0.40 & 29 & -2.87 & 0.34 & 0.77 & 0.3832 \\
\hline
\end{tabular}


Table 5. Results of one-way ANOVA testing for sexual dimorphism in dental relationships.

\begin{tabular}{|c|c|c|c|c|c|c|c|c|}
\hline \multirow[b]{2}{*}{ Variable } & \multicolumn{3}{|c|}{ Males } & \multicolumn{3}{|c|}{ Females } & \multirow[b]{2}{*}{ F Ratio } & \multirow[b]{2}{*}{ Prob $>\mathrm{F}$} \\
\hline & $\mathrm{n}$ & Mean & sem & $\mathrm{n}$ & Mean & sem & & \\
\hline Pretreatment Incisor Irreg. & 21 & 8.78 & 0.53 & 29 & 8.82 & 0.45 & 0.00 & 0.9505 \\
\hline Posttreatment Incisor Irreg. & 21 & 3.52 & 0.28 & 29 & 2.88 & 0.24 & 3.05 & 0.0874 \\
\hline Pretreatment Overjet & 21 & 5.48 & 0.43 & 29 & 4.64 & 0.37 & 2.16 & 0.1481 \\
\hline Posttreatment Overjet & 21 & 2.68 & 0.21 & 29 & 2.79 & 0.18 & 0.18 & 0.6746 \\
\hline Change in Overjet & 21 & -2.80 & 0.41 & 29 & -1.85 & 0.35 & 3.13 & 0.0831 \\
\hline Pretreatment Overbite & 21 & 3.41 & 0.39 & 29 & 2.77 & 0.33 & 1.58 & 0.2142 \\
\hline Posttreatment Overbite & 21 & 2.51 & 0.30 & 29 & 2.54 & 0.25 & 0.00 & 0.9477 \\
\hline Change in Overbite & 21 & -0.90 & 0.35 & 29 & -0.23 & 0.30 & 2.07 & 0.1568 \\
\hline Nance Space Needed & 21 & 102.79 & 1.31 & 29 & 98.71 & 1.12 & 5.60 & 0.0220 \\
\hline Nance Space Available & 21 & 100.68 & 1.66 & 29 & 95.54 & 1.41 & 5.58 & 0.0223 \\
\hline TSALD & 21 & -2.12 & 1.49 & 29 & -3.17 & 1.27 & 0.29 & 0.5929 \\
\hline
\end{tabular}


significantly larger in boys than girls $(\mathrm{P}=0.02)$. On the other hand, because both the required and the available perimeter sizes are larger in boys - and proportionately so-TSALD itself exhibits no suggestion of a sex difference.

\section{Extraction Space}

Extracting the first premolar in each maxillary quadrant should provide about $2 \times 7.2 \mathrm{~mm}=14.4 \mathrm{~mm}$ of space (Black 1902). A central issue in this study was to account for this extraction space-how much was used for canine retraction versus how much was "burned" by mesial movement of teeth in the buccal segment.

These relationships were determined by analyzing the teeth surrounding the extraction space in both the mesiodistal and transverse dimensions. These data indicate how much premolar extraction space a clinician hopes to maximize for the resolution of anterior crowding. The distal anatomic contact and the mesial anatomic contacts were marked from second molar to second molar in order to give and overall description of dental changes. The schematic plots (Figures A-1 through A-50) of all sample subjects are found in the appendix. Because many teeth contact each other along a broad zone of contact rather that at a one point contact, these points were marked in the middle of the anatomical contact zone. As a result, oftentimes these middle contact areas are not 
coincident even though the teeth are aligned sufficiently to place them in full intercuspation.

Table 6 lists the descriptive statistics for the orthodontic tooth movements. These data show that, on the average, the maxillary canine was retracted $3.8 \mathrm{~mm}$, while the second premolar was moved mesially $3.6 \mathrm{~mm}$. These movements sum to $7.3 \mathrm{~mm}$, which is the average mesiodistal diameter of a first premolar (Harris and Burris 2003).

These statistical averages obscure a good deal of inter-individual variation. Figures 4 through 7 show the actual sample distribution for the tooth movements listed in Table 5; and these graphs show the appreciable variability. For example, all cases in the sample experienced retraction of the maxillary canine into the premolar extraction site, but Figure 4 shows that less than $1 \mathrm{~mm}$ of canine retraction occurred in some patients, the modal retraction was on the order of 3.5 $\mathrm{mm}$, and most of the extraction space (in excess of $5.5 \mathrm{~mm}$ ) was used to reposition the canine at the upper extreme of the distribution. Just the converse is seen for the second premolar (Fig. 5). Less than $1 \mathrm{~mm}$ of mesial movement occurred in some patients, modal use was about $3.5 \mathrm{~mm}$, and as much as $5.5 \mathrm{~mm}$ of the extraction site was used to reposition the second premolar mesially at the other extreme. Inspection of the transverse changes in the canine (Fig. 6) and the second premolar (Fig. 7) also show appreciable ranges of variation, in excess of 5 $\mathrm{mm}$ for both variables. 
Table 6. Descriptive statistics for changes in tooth positions.

\begin{tabular}{lcccccc}
\hline Variable & $\mathrm{n}$ & Mean & $\mathrm{sd}$ & sem & $\mathrm{L}_{1}$ & $\mathrm{~L}_{2}$ \\
\hline \multicolumn{7}{c}{ Anteroposterior } \\
Distal U3 & 50 & 3.774 & 1.111 & 0.157 & 3.458 & 4.090 \\
Mesial U5 & 50 & -3.562 & 1.086 & 0.154 & -3.871 & -3.254 \\
& \multicolumn{7}{c}{ Transverse } \\
Distal U3 & 50 & -0.107 & 1.451 & 0.205 & -0.519 & 0.305 \\
Mesial U5 & 50 & -0.967 & 1.038 & 0.147 & -1.262 & -0.672 \\
\hline
\end{tabular}

Note: Left and right sides have been averaged on a case-by-case basis. 


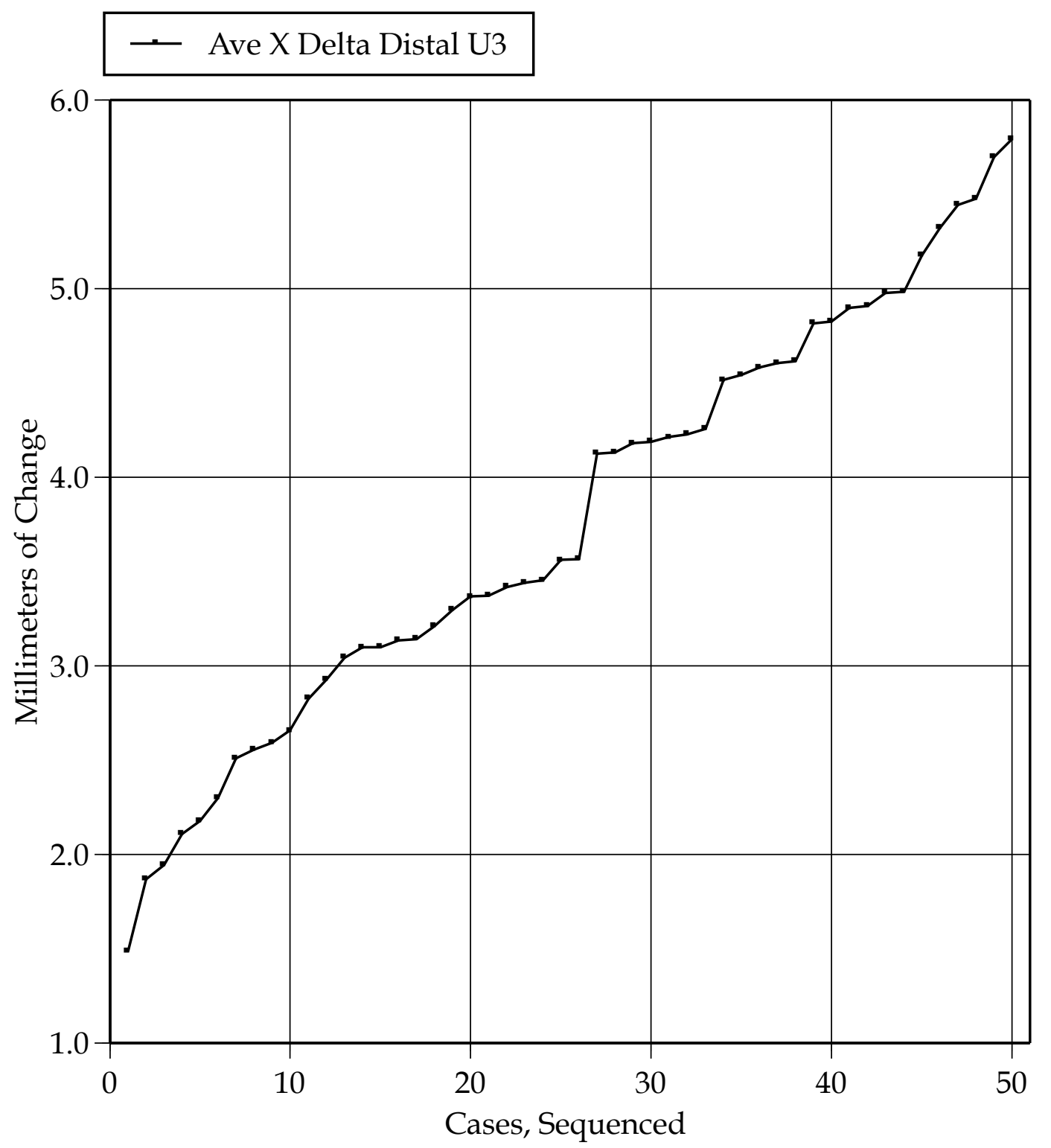

Fig. 4. Sequenced array of cases according to the amount of observed mesiodistal change in the distal contact of the maxillary canine There are 50 cases in the sample, so the median change is at case 25. Left and right sides were averaged for each case. Positive values represent distal movement 


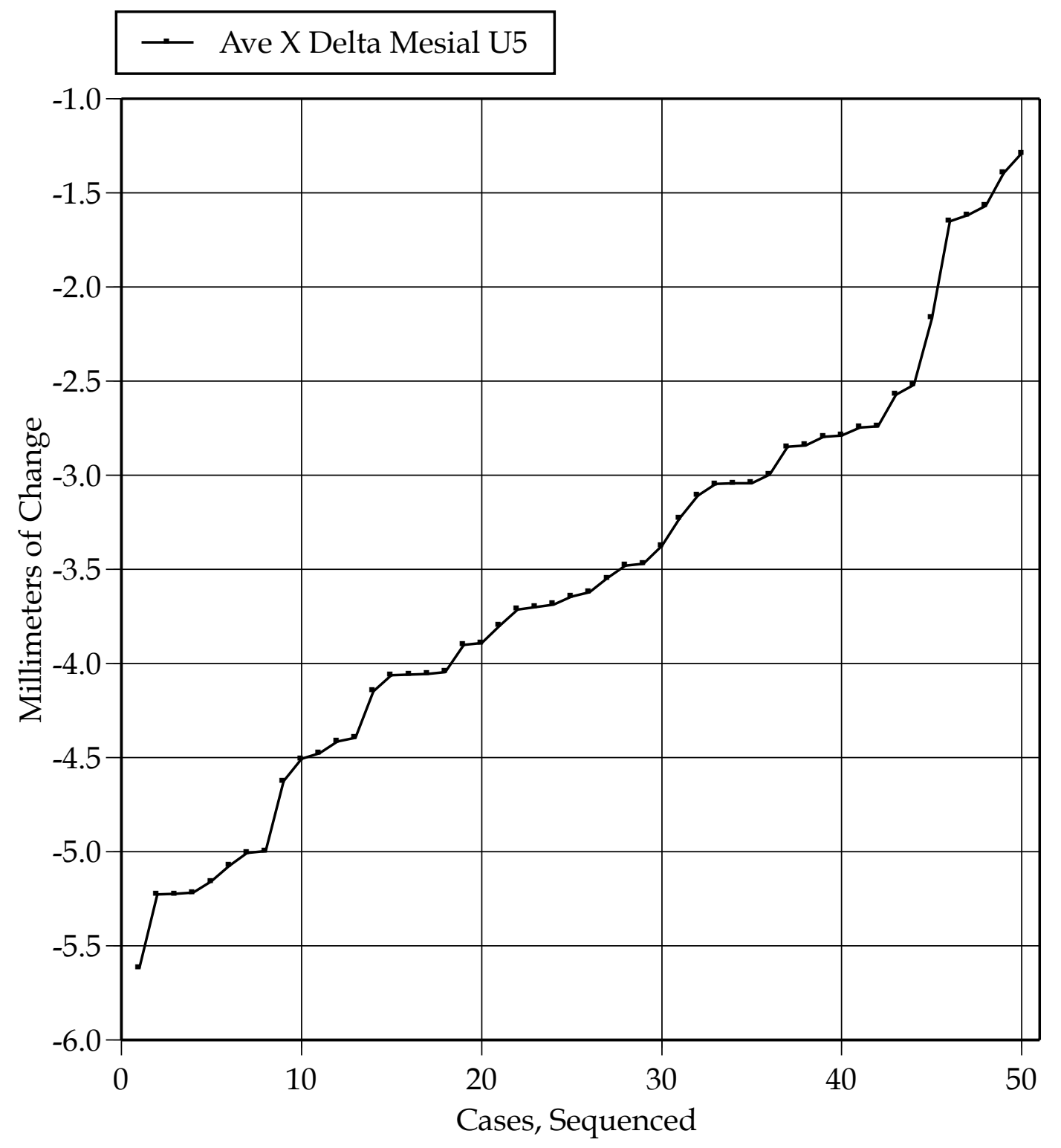

Fig. 5. Sequenced array of cases according to the amount of observed mesiodistal change in the mesial contact of the maxillary second premolar. There are 50 cases in the sample, so the median change is at case 25 . Left and right sides were averaged for each case. Negative values represent mesial movement. 


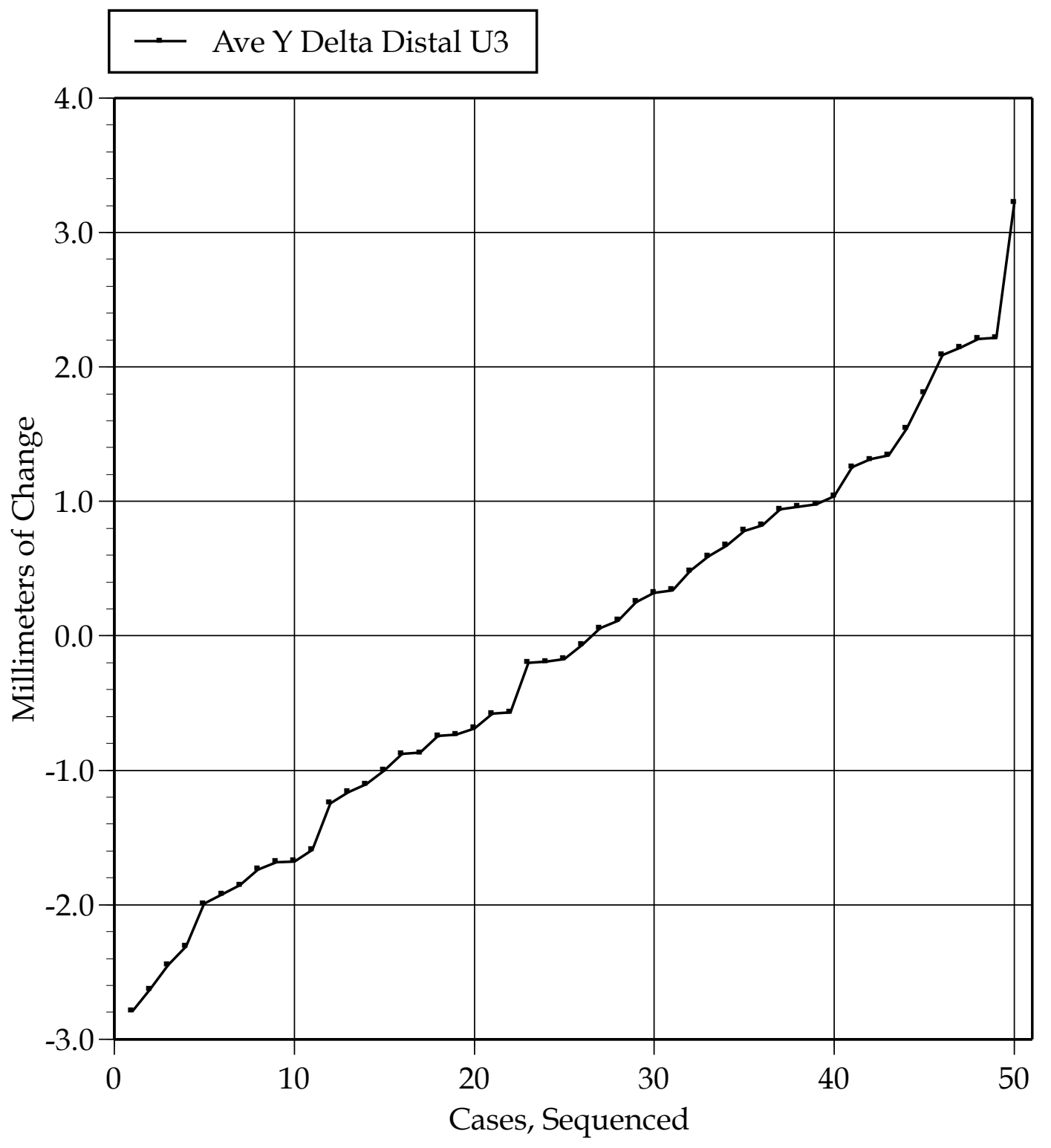

Fig. 6. Sequenced array of cases according to the amount of observed transverse change in the distal contact of the maxillary canine. There are 50 cases in the sample, so the median change is at case 25. Left and right sides were averaged for each case. Positive values represent lateral movement. 


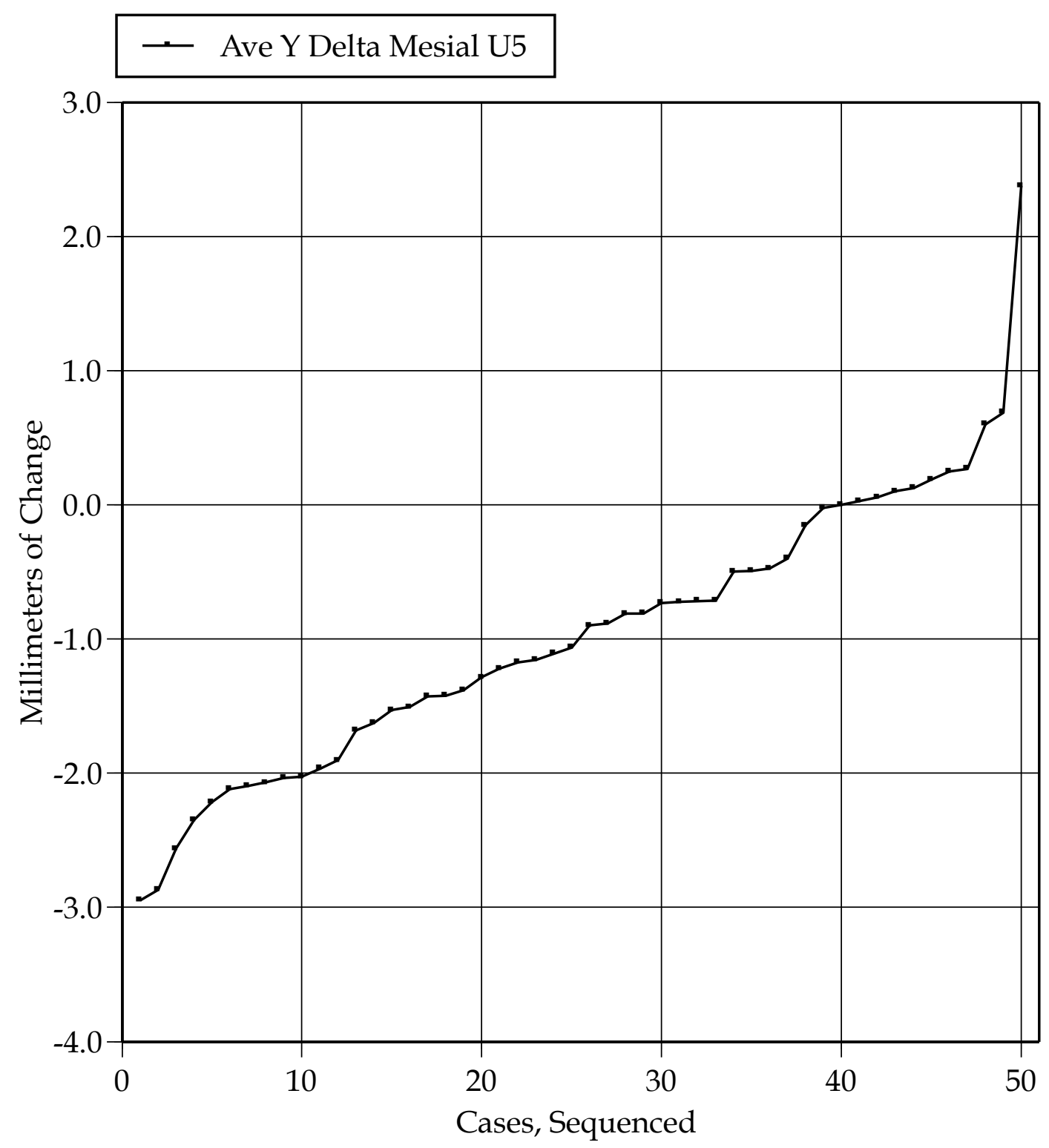

Fig. 7. Sequenced array of cases according to the amount of observed transverse change in the mesial contact of the maxillary second premolar. There are 50 cases in the sample, so the median change is at case 25 . Left and right sides were averaged for each case. Positive values represent expansion. 


\section{Use of the Extraction Space}

At its simplest, we can see from the sample statistics (Table 6) that right at half of the first premolar extraction space was used to retract the canine and the other half was used to move the buccal segment mesially. That is, of the $7.3 \mathrm{~mm}$ of extraction space, $51 \%$ was used to move the canine distally and the other $49 \%$ was used to move the buccal segment mesially. Of course, this modal solution is essentially uninteresting and uninformative.

It was of more clinical relevance to discern whether the amount of canine retraction could be predicted from aspects of the malocclusion. It is pretty well established that first premolar extractions are preferred when a major component of the malocclusion is near at hand, such as incisor irregularity or incisor procumbency (including bimaxillary excess). These are, for example, the harbingers suggested in popular orthodontic textbooks (e.g., Moyers 1973; Proffit 2000).

As detailed in the Methods section, we collected data on so-called "predictor variables" that might be predictive in the statistical sense of how much canine retraction was required. These variables were (1) maxillary incisor irregularity, (2) incisor overjet, (3) incisor overbite, and (4) Nance's TSALD. The issue was whether the pretreatment status of these predictor variables could be used, singly or in combination, to predict the amount of canine retraction. The anteroposterior change in canine position was the independent variable, and 
potential predictors were the dependent variables in this series of bivariate tests (Table 7). The ease of computing these tests led us to include some dependent variables that were not likely to be predictive.

Two variables were significantly associated with the amount of canine retraction into the extraction site. One was the severity of maxillary incisor irregularity at the start of treatment $\left(\mathrm{r}^{2}=0.11 ; \mathrm{P}=0.02\right)$. The other was the amount of transverse (buccolingual) change in the canine's position $\left(\mathrm{r}^{2}=0.18 ; \mathrm{P}<\right.$ 0.01). This first significant association is intuitively obvious. One supposes that the severity of crowding (incisor irregularity) in the anterior segment would influence the orthodontist's decision to extract first premolars and use the extraction space to "unravel" the maloccluded anterior teeth. As shown in Figure 8 , the nature of the association is that the greater the irregularity the more the canines were retracted. Statistically, however, this relationship is not strong, explaining only about $10 \%$ of the variation. The regression coefficient shows that $1 \mathrm{~mm}$ of canine retraction (in each of the two quadrants) corresponds to the alleviation of $0.72 \mathrm{~mm}$ of irregularity.

The other significant relationship disclosed in Table 7 was not as apparent. There is a significant relationship between the amount of canine retraction and the amount transverse correction of this same tooth $(\mathrm{P}<0.01)$. Indeed, with an adjusted $\mathrm{r}^{2}$ of $17 \%$, this is readily the strongest relationship detected. The bivariate plot (Fig. 9) shows that cases with the least canine retraction 
Table 7. Results of linear regression predicting the amount of canine retraction.

\begin{tabular}{|c|c|c|c|c|c|c|c|}
\hline $\begin{array}{c}\text { Dependent } \\
\text { Variable }\end{array}$ & $\mathrm{r}^{2}$ & Adjusted $\mathrm{r}^{2}$ & $\begin{array}{l}\text { RMS } \\
\text { Error }\end{array}$ & $\begin{array}{l}\text { Regression } \\
\text { Coefficient }\end{array}$ & St Error & t-test & Prob $>|t|$ \\
\hline Pretreatment Irregularity & 0.1108 & 0.0923 & 2.2774 & 0.7160 & 0.2928 & 2.45 & 0.0182 \\
\hline Posttreatment Irregularity & 0.0143 & -0.0062 & 1.3178 & -0.1415 & 0.1694 & -0.84 & 0.4077 \\
\hline Pretreatment Overjet & 0.0006 & -0.0203 & 2.0305 & -0.0426 & 0.2610 & -0.16 & 0.8712 \\
\hline Posttreatment Overjet & 0.0427 & 0.0228 & 0.9330 & 0.1755 & 0.1199 & 1.46 & 0.1498 \\
\hline Change in Overjet & 0.0160 & -0.0045 & 1.9216 & 0.2181 & 0.2470 & 0.88 & 0.3817 \\
\hline Pretreatment Overbite & 0.0190 & -0.0015 & 1.7866 & 0.2214 & 0.2297 & 0.96 & 0.3399 \\
\hline Posttreatment Overbite & 0.0103 & -0.0103 & 1.3474 & 0.1223 & 0.1732 & 0.71 & 0.4834 \\
\hline Change in Overbite & 0.0045 & -0.0162 & 1.6462 & -0.0990 & 0.2116 & -0.47 & 0.6419 \\
\hline Space Required & 0.0109 & -0.0097 & 6.3267 & 0.5922 & 0.8133 & 0.73 & 0.4701 \\
\hline Space Available & 0.0106 & -0.0100 & 7.9751 & -0.7354 & 1.0252 & -0.72 & 0.4766 \\
\hline TSALD & 0.0475 & 0.0277 & 6.6748 & -1.3276 & 0.8580 & -1.55 & 0.1284 \\
\hline $\begin{array}{l}\text { Transverse Change at } \\
\text { Second Premolar } \\
\text { Transverse Change at }\end{array}$ & 0.0001 & -0.0207 & 1.0488 & 0.0108 & 0.1348 & 0.08 & 0.9364 \\
\hline Canine & 0.1845 & 0.1675 & 1.3235 & -0.5607 & 0.1701 & -3.30 & 0.0019 \\
\hline
\end{tabular}




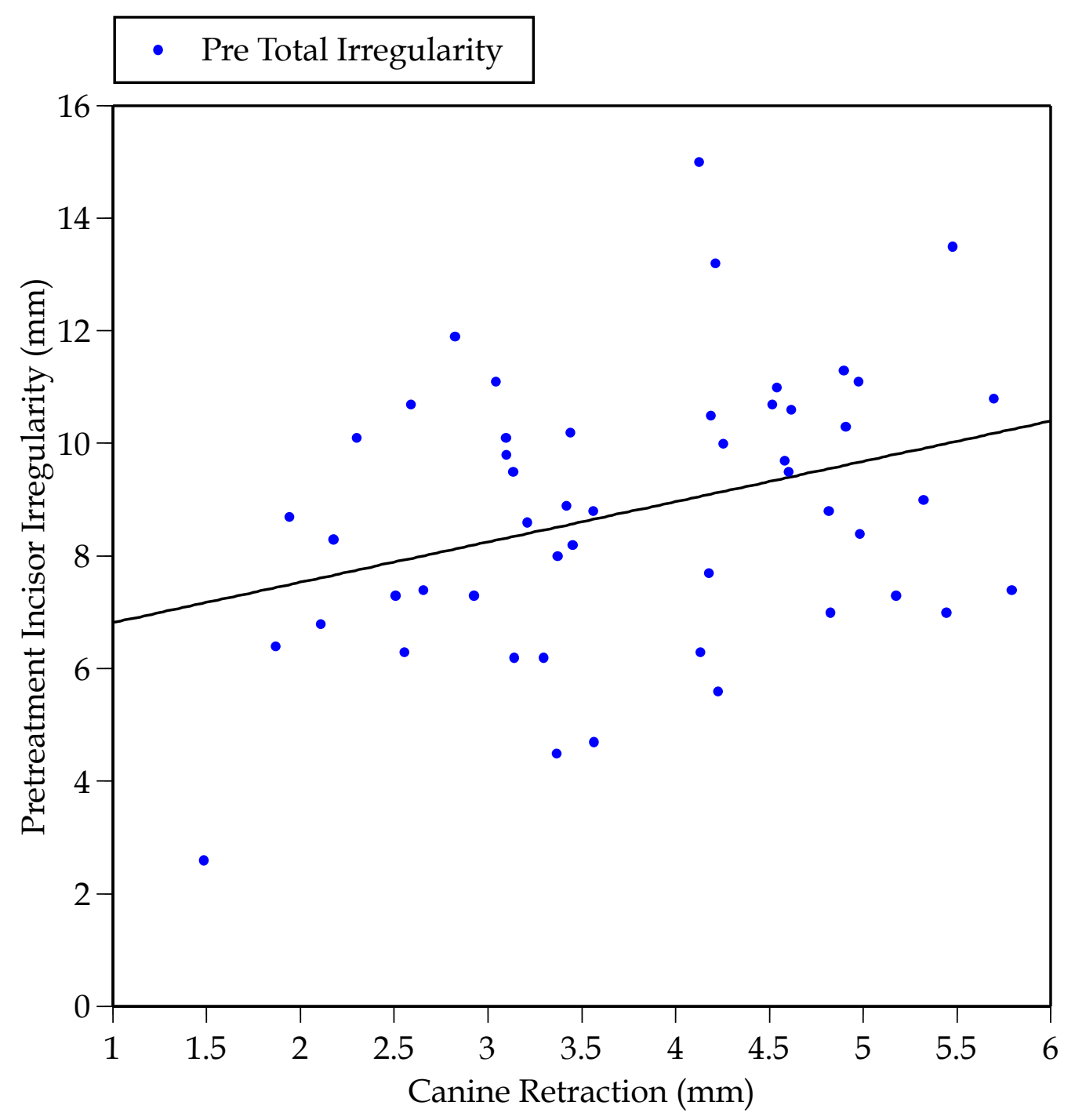

Fig. 8. The association between the amount of canine retraction and the degree of maxillary incisor irregularity at pretreatment. The correlation coeffient between variables is 0.33 . 


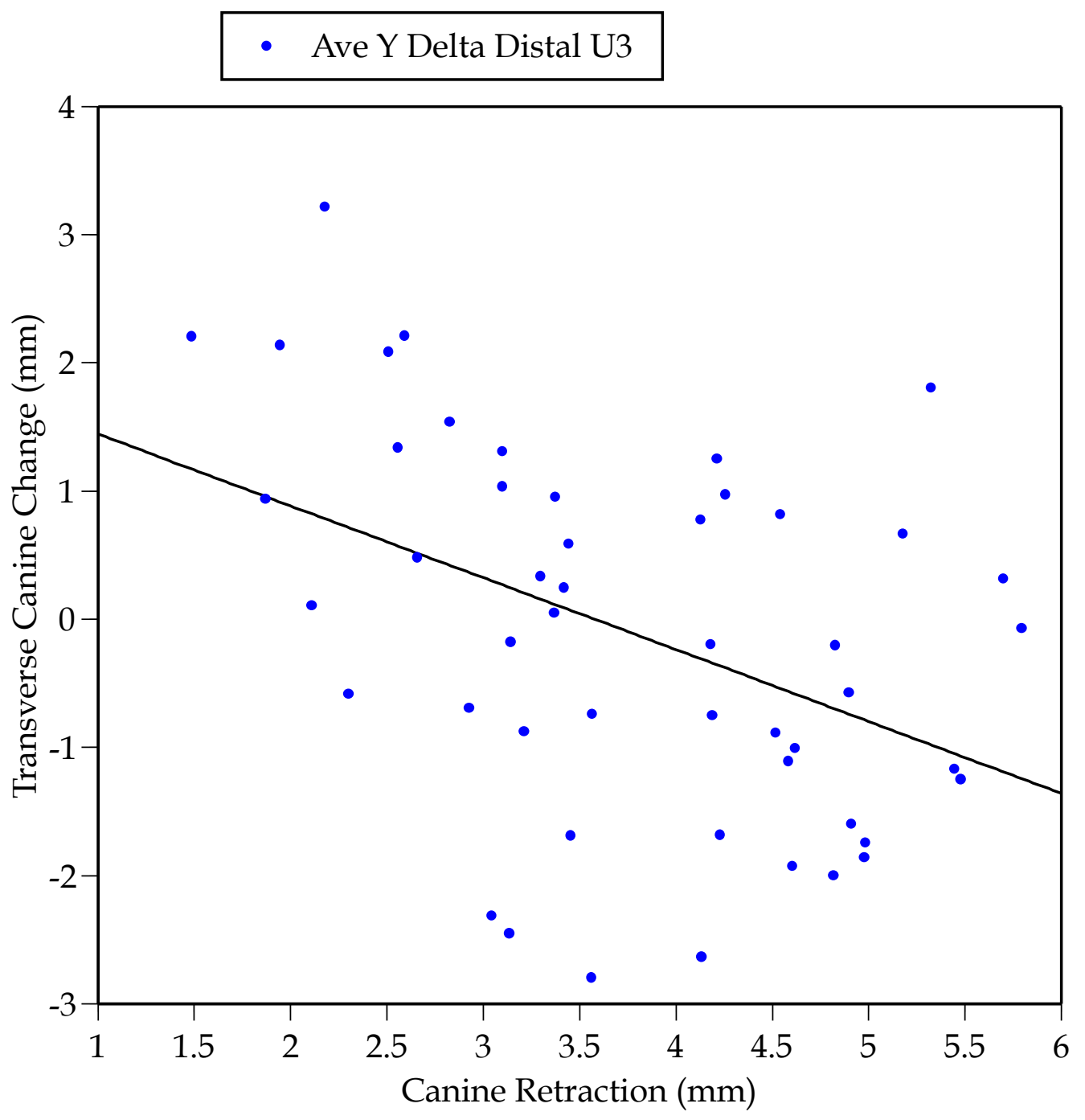

Fig. 9. The association between the amount of canine retraction and the amount of transverse change in canine position. the correlation coefficient is 0.43 with an associated r-square of $18 \%$. 
experienced the most canine expansion. Conversely, where there was considerable canine retraction, there tended to be reduction in intercanine width. Inspection of a few of the extreme cases makes the clinical requirements of these changes apparent. First, picking two cases at the upper-left extreme of the distribution (cases DB and TS, Figs. A-14 and A-48) we see that there is little anterior crowding; not much additional space is required to align the anterior teeth. There is, then, little canine retraction, though the intercanine width is expanded to "round-out" the arch form and reduce the overjet.

Cases LK and DG (Figs. A-32 and A-13) are at the lower-right extreme of the distribution in Figure 9. In these cases (1) there is appreciable anterior crowding, (2) the canines are blocked-out buccally, and (3) the lateral incisors are positioned lingual to the canines. Collectively, then, the canines need to be retracted a goodly amount (using up much of the first premolar extraction space) in order to move the lateral incisors into alignment.

At the two extremes just looked at, little canine retraction is needed when the anterior teeth are already in alignment, while considerable canine retraction is needed to accommodate blocked-out canines along with considerable anterior crowding - and this is the gist of the association shown graphically in Figure 9.

Table 7 also discloses some interesting "non-associations." For example, we had supposed that first premolar extractions would by associated with overjet - that excessive overjet would be resolved in part by retracting the 
anterior segment. This was not the case $(\mathrm{P}=0.87)$. So too, Nance's TSALD was of no predictive value in explaining the amount of canine retraction $(\mathrm{P}=0.13)$.

\section{$\underline{\text { Multivariate Model }}$}

It is reasonable to suppose that some combination of predictors would account for more of the variation than any taken singly (e.g., Draper and Smith 1966; Cohen and Cohen 1975). This was explored here using stepwise multiple linear regression (Freund and Littell 1991).

Model building began with 12 potential predictors, namely (1) incisor irregularity at pretreatment, (2) transverse canine change, (3) transverse second premolar change, (4) space required at pretreatment, (5) space available at pretreatment, (6) TSALD at pretreatment, (7) overjet at pretreatment, (8) overjet at posttreatment, (9) change in overjet, (10) overbite at pretreatment, (11) overbite at posttreatment and (12) change in overbite. Using the forward-stepping procedure and the conventional level of alpha (0.05) for variable retention, just two predictors were entered. (Other selection criteria were tested, but these results were robust.) The two variables were (1) transverse canine change and (2) incisor irregularity at pretreatment (Table 8). Quite as suggested by the univariate results above, the most influential predictor is the transverse change in canine position, with a negative regression coefficient, followed by severity of incisor irregularity. Collectively, the model has modest predictive value, with an 
Table 8. Results of stepwise linear regression predicting the amount of canine retraction.

\begin{tabular}{lcccc}
\hline \multicolumn{1}{c}{ Term } & $\begin{array}{c}\text { Regression } \\
\text { Coefficient }\end{array}$ & Std Error & t-test & Prob $>|\mathrm{t}|$ \\
\hline Intercept & 2.4648 & 0.5245 & 4.70 & $<0.0001$ \\
Pretreatment Irregularity & 0.1448 & 0.0576 & 2.52 & 0.0154 \\
Transverse Canine Change & -0.3167 & 0.0949 & -3.34 & 0.0017 \\
\hline
\end{tabular}


adjusted $\mathrm{r}^{2}$ of $25 \%$. The model F-ratio was $9.2(\mathrm{P}=0.0004)$. In other words, while two significant predictors of the amount of canine retraction were identified, the bulk of the variation remains unexplained. 


\section{CHAPTER V}

\section{DISCUSSION}

The magnitude of tooth movements surrounding first premolar extraction sites were used in the present study to determine how the space gained by extracting four first premolars is used by the orthodontist in resolving patients' malocclusions. A common question is how much of the extraction space is used to resolve anterior crowding and how much of it will be consumed as the buccal segment moves mesially. There are many reasons for a clinician to choose tooth extraction as a means of correcting the malocclusion. Characteristics like anterior or posterior crowding, Class II molar and canine relationships, facial height factors, habits, can all be factors involved in making this decision (e.g., Schoppe 1964; Moyers 1973; Baumrind et al. 1996; Proffit 2000).

A sample of 50 adolescent North American whites with pretreatment Class I molar malocclusions were selected for the study. In all cases, four first premolars were extracted and the buccal segments were maintained in a Class I relationship.

In a sample consisting just of Class I malocclusions, it is a reasonable assumption that most of the extraction space will be used to resolve anterior malocclusion factors such as crowding, overbite, overjet, and protrusion 
(Schoppe 1964; Graber 1972; Dewel 1973; Moyers 1973; Proffit 2000). The

extraction space will not be needed to resolve buccal segment relationships as the sample patients already were Class I.

\section{$\underline{\text { Side Differences }}$}

As anticipated, the differences between the two sides as given by the repeated measures ANOVA tests were so minor that we combined the two sides for the rest of the analyses. However, instead of describing a clear symmetrical movement of canines or second premolars, the data disclosed a significant asymmetrical movement in the transverse dimension wherein the left side experienced mean expansion while the right side was constricted overall. One possibility is that these significant differences are related to the handedness of the clinician. Because the majority of humans are right-handed, one assumes that the majority of orthodontists and assistants are right handed also. This means that the majority of patients are treated by someone who is oriented to their right. As a result, the way in which an archwire is repeatedly inserted from the patient's right side could cause an overall increase in constriction on the right side as compared to the left side. While these differences were clinically minor, their detection by this unique measurement protocol brings to question how much influence the handedness of the clinician has on the final arch form. 


\section{$\underline{\text { Sexual Dimorphism }}$}

The majority of the tests for sexual dimorphism were nonsignificant statistically. It is hard to decipher why more differences did not exist between the sexes because of many factors including malocclusion severity, compliance issues and tooth size.

Females seek orthodontic treatment at roughly a two-to-one ratio compared to males (Keim et al. 2002.) However, epidemiological studies have shown that severity of malocclusion is not significantly different between males and females (Kelly and Harvey 1977; Proffit, Fields and Moray 2000). Thus, malocclusion severity differences can produce a sexual bias because girls (and their parents) seek treatment for less severe malocclusions than boys. In other words, if girls want treatment for trivial malocclusions more commonly than boys, the extraction space needed to resolve these malocclusions would be less. One would expect slightly more intentional anchorage slippage as the clinician closes the remaining unneeded space at the end of treatment. However, one would expect to see a corresponding decrease in canine retraction amounts if this were true as well as significantly different overjet and overbite amounts between males and females before treatment. The data did not show these associated characteristics; while males did have greater pretreatment overjet and overbite, it was not to a significant degree. 
Concerning compliance, increased female compliance over males could account for differences in the mesial movement of the buccal segments as headgear or other auxiliaries could have been used to restrain the movement of the posterior segments. Egolf, BeGole, and Upshaw (1978) reported that three of eight studies comparing compliance levels between sexes have shown an increased compliance level for females over males, while the remaining five showed no difference. None of the studies that they evaluated showed males to exhibit greater compliance than females. Compliance levels or treatment auxiliaries were not considered in this study so any effect remains speculative.

Tooth size differences can also contribute to the discrepancies between the sexes. According to tooth size data from this study, girls in the sample had an average $0.3 \mathrm{~mm}$ smaller first premolar tooth size than boys as measured from the mesial contact to the distal contact. Smaller first premolars obviously result in smaller extraction spaces than in boys, so the quantity of potential movement of the second premolars would be diminished from the inception of treatment.

Pretreatment TSALD measurements revealed that girls had $1.1 \mathrm{~mm}$ more crowding than boys in this sample. This seemingly important difference failed to reach significance because of large outliers among the boys that resulted in a standard error (2.2) which was larger than the mean male TSALD measurements themselves. 
Larger corrections of overjet and overbite were seen among boys than among girls. This difference is easily explained by a comparison of pretreatment overjet and overbite. Before treatment, boys had $0.8 \mathrm{~mm}$ more overjet on average and $0.6 \mathrm{~mm}$ more overbite on average than girls. So, more correction occurred in boys because they needed more in order to achieve a proper anterior relationship.

\section{Extraction Space}

\section{Mesiodistal Dimension}

Removing a first premolar gave an average of $7.3 \mathrm{~mm}$ of space mesiodistally in each quadrant when calculated by combining canine and second premolar movement amounts. Of this space, $3.8 \mathrm{~mm}(51 \%)$ of it was consumed by canine retraction, while $3.6 \mathrm{~mm}(49 \%)$ was used as the second premolar moved mesially. These values give us a picture that first premolar extraction spaces are used up roughly half and half by retraction and buccal segment advancement, respectively. Cases NL and OS (Figs. A-39 and A-40) give a good example of this equal use of space. The distal of the canines at posttreatment is roughly coincident with the middle of the pretreatment first premolar position. This fairly even split in the way the extraction space is used is different from results published by Williams and Hosila (1976) who stated that $67 \%$ of the space was taken by retracted anterior segments using the Begg technique with no 
headgear. Similarly, Creekmore (1976) stated that two-thirds of the extraction space could be used for retraction and the correction of crowding and only onethird would be used by the buccal segments. Overall, previous studies have generally shown more maxillary canine retraction than was observed in this study (Steyn, du Preez and Harris 1997; Ong and Woods 2001), but several of these studies were including Class II patients in their sample and not just Class I patients. The canine and buccal segment relationship in Class II patients requires increased use of the extraction space for anterior retraction in order to correct to a Class I. Therefore, it is predictable that we would see less canine retraction occurring in the present study that was specifically composed of just Class I patients. These cases most likely needed extraction space only to resolve anterior crowding instead of also needing canine and buccal segment interarch correction. Thus, the orthodontists treating them would not need to take full advantage of the extraction space for canine retraction unless the patient presented with a soft tissue imbalance like a bimaxillary protrusion.

Two cases that illustrate increased canine retraction to compensate for dentoalveolar protrusion are LR and TM (Figs. A-4 and A-49). These cases are both on the higher end of the canine retraction spectrum. However, it can be noted by looking at the figures, that while both patients had only mild to moderate crowding, the canines were retracted a large amount resulting in 
significant retraction of the anterior segments to compensate for the soft tissue imbalance.

It has been mentioned that there was no real discrepancy between the calculated extraction space $(7.3 \mathrm{~mm})$ and the actual mesiodistal measurements of the first premolars prior to treatment $(7.2 \mathrm{~mm})$. What difference does exist can be accounted for by pretreatment rotations of the first premolars. While these teeth can be measured from mesial contact point to distal contact point in order to predict mesiodistal extraction space, the actual extraction space will always be slightly greater. This is because the rotation of teeth will consume more room in the arch mesiodistally than their actual anatomical mesiodistal width.

\section{Transverse Dimension}

Changes in the transverse dimension after premolar extraction treatment were minor. When measured against positions of the palatal rugae, the canines moved almost straight back with less than $1 \mathrm{~mm}$ difference from their pretreatment transverse positions. The second premolars did constrict significantly from pretreatment to posttreatment, an average of $1 \mathrm{~mm}$. However, this increased amount of constriction must be viewed in the context that they have moved mesially to contact the canines that did not change systematically in their transverse positions. These findings would seem to neither support the "collapse theory" that suggests that premolar extractions cause a constriction of 
the arches nor the "expansion theory" that purports that canines are retracted into the broader arch diameter where the premolars had been located (Bishara et al. 1994; Vaden, Harris and Gardner 1997). While the constriction of arches following premolar extraction has been mainly anecdotal, several studies have shown that expansion can occur in the arches following extractions (Bishara et al. 1994; O'Higgins and Lee 2000; Gianelly 2003). The limitation of these studies is that the samples are not comparable to that of the present study. They combined Class I, II, and III patients in some instances, and there is no way to know what amount of transverse change was needed in the first place. Were these patients in posterior crossbite? Was an expansion appliance used in the maxillary arch? Gianelly's study is especially ambiguous because it was not done clinically, but rather treatment was simulated on orthodontic casts. The data reported in our study suggest that the teeth stay essentially where they were to begin with in the transverse dimension. The buccal corridor space, considered unaesthetic, would therefore not become larger as a result of extraction, or smaller as the anterior teeth are retracted into a broader diameter.

\section{Predictive Models}

This research attempted to quantify what pretreatment characteristics would lead a clinician to select for the extraction of four first premolars as part of a patient's treatment. Surprisingly, out of these twelve factors tested, only 
transverse canine change and incisor irregularity showed a significant relationship with the amount of mesiodistal canine retraction. Neither of these variables accounted for a large part of the variation, so their predictive value, especially clinically, is modest.

Transverse canine position as a reason for choosing extractions is not mentioned specifically in the literature although some studies have shown it to be a sequela to extraction therapy (Bishara et al. 1994; O'Higgins and Lee 2000; Gianelly 2003).

A positive association was seen as the amount of canine retraction increased as the pretreatment incisor irregularity increased. This connection is intuitive as a clinician would want more retraction of the canines to resolve an increased amount of crowding. This shows the importance of anterior crowding as Little's (1975) analysis measures the differences in the contact areas resulting from crowding and tooth rotations. However, it is counterintuitive that this same relationship was not seen in the overjet, overbite, or TSALD variables to a significant level. TSALD in particular has been reported to be a major factor in making an extraction decision (Baumrind et al. 1996). 


\section{CHAPTER VI}

\section{SUMMARY AND CONCLUSIONS}

A critical historical issue in orthodontics concerns the use of premolar extractions to resolve discrepancies between the mesiodistal tooth crown dimensions and the bony arch perimeter. Virtually all the luminaries in orthodontic literature including Angle have grappled with this issue in some degree-many of them coming to different conclusions about premolar extraction indications and results (Proffit 2000).

Considerable research has been devoted to the effects of premolar extraction. The majority of these studies used cephalometrics to evaluate anteroposterior change in the dental arch (e.g., Williams and Hosila 1976; Carter 1988; Bishara et al. 1995), while only a few have used dental casts to investigate transverse changes as well (e.g., Cusimano, McLaughlin and Zernik 1993; Ong and Woods 2001).

Regarding premolar extraction decisions, only a few studies have tried to statistically quantify what characteristics of the malocclusion lead a clinician to select for premolar extractions as a treatment option (e.g., Baumrind et al. 1996). Most literature that examines why and when extractions should be done only provides anecdotal reasons for this treatment option based on the author's 
experience or preferences (e.g., Tweed 1944; Schoppe 1964; Dewel 1973;

Creekmore 1997). Similarly, broad guidelines are given in textbooks about when to extract, but little statistical analysis is usually cited to defend these claims (e.g., Graber 1972; Moyers 1973; Proffit 2000).

The purpose of the present study was to characterize how the first premolar extraction space was used, that is, how much of the space was used for retraction of the canine (to alleviate crowding and/or protrusion in the anterior segment) versus how much was used to adjust the buccal segment. We developed a novel three-dimensional coordinate analysis to study these changes. Dental casts from a Class I adolescent group treated with four first premolar extractions were analyzed with a three dimensional digitizing system using the distal palatal rugae as fiducial landmarks. The shortcoming of most prior studies is that their sample groups were not homogenous, but rather a combination of Class I and Class II patients. The present study was restricted to a sample of Class I patients. Anteroposterior and mediolateral changes of all teeth were analyzed. (The comparatively minor vertical changes were ignored here.) Precision and repeatability accuracy were very satisfactory using this protocol. 
Major findings of the effects and reasons for premolar extractions are summarized here:

- A four first premolar extraction pattern in Class I patients provides space that is consumed almost equally by the retraction of canines (51\%) and the mesial movement of the buccal segments in the maxilla (49\%).

- Transverse changes after extraction were minor, although arch width at the second premolars diminished approximately $1 \mathrm{~mm}$.

- Incisor irregularity had a significant positive relationship with the amount of canine retraction.

- Multivariately, the two significant variables associated with the decision to extract in these patients were (1) transverse canine change and (2) a high level of incisor irregularity. When canines are crowded out of the arch and have to move substantially, this is predictive of greater canine retraction. However, these variables did not represent the majority of predictive variables because they had a low $\mathrm{r}^{2}$ value.

In sum, the present study shows that in Class I patients, canine retraction amounts after first premolar extraction are slightly less than previous studies have shown (e.g., Williams and Hosila 1976; Creekmore 1997). Transverse changes appear to depend on the amount of retraction that is accomplished, with a constriction of the arch occurring with increased retraction and expansion of the arch occurring with decreased retraction. 
The main variables associated with the decision to extract were incisor irregularity and transverse canine change. The data also described an intuitive relationship between large tooth size-arch length discrepancies and large amounts of canine retraction. 
LIST OF REFERENCES 
Almeida MA, Phillips C, Kula K, Tulloch C. Stability of the palatal rugae as landmarks for analysis of dental casts. Angle Orthod 1995;65:43-8.

Ashmore JL, Kurland BF, King GJ, Wheeler TT, Ghafari J, Ramsay DS. A 3dimensional analysis of molar movement during headgear treatment. Am J Orthod Dentofacial Orthop 2002;121:18-30.

Bailey LJ, Esmailnejad A, Almeida MA. Stability of the palatal rugae as landmarks for analysis of dental casts in extraction and nonextraction cases. Angle Orthod 1996;66:73-8.

Baume LJ, Horowitz HS, Summers CJ, Dirks BO, Brown WAB, Carlos JP, Cohen LK, Freer TJ, Harvold EP, Moorrees CFA, Salzmann JA, Schmuth G, Solow B, Taatz H. A method for measuring occlusal traits. Int Dent J 1973;23:530-7.

Baumrind S. Commentary [In Ashmore et al. A 3-dimensional analysis of molar movement during headgear treatment]. Am J Orthod Dentofacial Orthop 2002;121:29-30.

Baumrind S, Korn EL, Boyd RL, Maxwell R. The decision to extract: part II. Analysis of clinicians' stated reasons for extraction. Am J Orthod Dentofacial Orthop 1996;109:393-402.

Begg PR, Kesling PC. Begg orthodontic theory and technique, 3rd ed. Philadelphia: WB Saunders, 1977. 
BeGole EA. Application of the cubic spline function in the description of dental arch form. J Dent Res 1980;59:1549-56.

Bishara SE, Bayati P, Zaher AR, Jakobsen JR. Comparisons of the dental arch changes in patients with Class II, division 1 malocclusions: extraction vs nonextraction treatments. Angle Orthod 1994;64:351-8.

Bishara SE, Cummins DM, Jakobsen JR, Zaher AR. Dentofacial and soft tissue changes in Class II, division 1 cases treated with and without extractions. Am J Orthod Dentofacial Orthop 1995;107:28-37.

Bishara SE, Cummins DM, Zaher AR. Treatment and posttreatment changes in patients with Class II, division 1 malocclusion after extraction and nonextraction treatment. Am J Orthod Dentofacial Orthop 1997;111:18-27.

Black GV. Descriptive Anatomy of the Human Teeth, 2nd ed. Philadelphia: Wilmington Dental Manufacturing Company, 1902.

Bolton WA. Disharmony in tooth size and its relation to the analysis and treatment of malocclusion. Angle Orthod 1958;28:113-30.

Brandt S, Safirstein GR. Different extractions for different malocclusions. Am J Orthod 1975;68:15-41.

Cassidy KM. Heritability estimates of arch form: a sibling analysis. Unpublished Master's thesis, University of Tennessee, Memphis, 1996.

Carter NE. First premolar extractions and fixed appliances in the Class II division 1 malocclusion. J Orthod 1988;15:1-10. 
Cohen J, Cohen P. Applied multiple regression/correlation analysis for the behavioral sciences. New York: John Wiley and Sons, 1975.

Creekmore TD. Where teeth should be positioned in the face and jaws and how to get them there. J Clin Orthod 1997;31:586-608.

Cusimano C, McLaughlin RP, Zernik JH. Effects of first bicuspid extractions on facial height in high-angle cases. J Clin Orthod 1993;27:594-8.

Darendeliler N, Taner-Sarisoy L. The influence of orthodontic extraction treatment on dental structures: a two-factor evaluation. Eur J Orthod 2001;23:295-303.

De Castro N. Second premolar extraction in clinical practice. Am J Orthod $1974 ; 65: 115-37$.

Dewel, BF. Second premolar extraction in orthodontics: principles, procedures, and case analysis. Am J Orthod 1955;41:107-20.

Dewel, BF. Extraction in orthodontics: premises and prerequisites. Angle Orthod 1973;43:65-87.

Draper NR, Smith H. Applied regression analysis. New York: John Wiley and Sons Inc, 1966.

English WR, Robison SF, Summitt JB, Oesterle LJ, Brannon RB, Morlang WM. Individuality of human palatal rugae. J Forensic Sci 1988;33:718-26. 
Egolf RJ, BeGole EA, Upshaw HS. Factors associated with orthodontic patient compliance with intraoral elastic and headgear wear. Am J Orthod 1990;97:336-48.

Freund FJ, Littel RC. SAS system for regression, 2nd ed. Cary, NC: SAS Institute Inc., 1991.

Gianelly AA. Arch width after extraction and nonextraction treatment. Am J Orthod Dentofacial Orthop 2003;123:25-8.

Graber TM. Orthodontics: principles and practice, 3rd ed. Philadelphia: WB Saunders Company, 1972.

Harris EF, Burris BG. Contemporary permanent tooth dimensions, with comparisons to G. V. Black's data. J Tenn Dent Assoc 2003;83:25-9.

Hoggan BR, Sadowsky C. The use of palatal rugae for the assessment of anteroposterior tooth movements. Am J Orthod Dentofacial Orthop 2001;119:482-8.

Jepsen A. Root surface measurement and a method for x-ray determination of root surface area. Acta Odontol Scand 1963;21:35-46.

Keim RG, Gottlieb EL, Nelson AH, Vogels DS. 2002 JCO study of orthodontic diagnosis and treatment procedures. Part 1. Results and trends. J Clin Orthod 2002;36:553-68. 
Kelly JE, Harvey CR. An assessment of the occlusion of youths 12-17 years. United States Public Health Service, 1977 (Vital and health statistics; series 11; no. 162).

Kocadereli I. The effect of first premolar extraction on vertical dimension. Am J Orthod Dentofacial Orthop 1999;116:41-5.

Little RM. The irregularity index: a quantitative score of mandibular anterior alignment. Am J Orthod 1975;68:554-6.

Logan LR. Second premolar extraction in Class I and Class II. Am J Orthod $1973 ; 63: 115-47$.

Lysell L. Plicae palatinae transverse and papilla incisiva in man: a morphologic and genetic study. Acta Odont Scand 1955;13:106-33.

$\mathrm{Lu} \mathrm{KH}$. An orthogonal analysis of the form, symmetry and asymmetry of the dental arch. Arch Oral Biol 1966;11:1057-69.

Luppanapornlarp S, Johnston LE. The effects of premolar-extraction: a long-term comparison of outcomes in "clear-cut" extraction and nonextraction Class II patients. Angle Orthod 1993;63:257-72.

Moyers RE. Handbook of orthodontics, 4th ed. Chicago: Year Book Medical Publishers, Inc., 1973.

Nance HN. The limitations of orthodontic treatment, II. Am J Orthod 1947;33:253-301. 
O'Higgins EA, Lee RT. How much space is created from expansion or premolar extraction? J Orthod 2000;27:11-3.

Ong HB, Woods MG. An occlusal and cephalometric analysis of maxillary first and second premolar extraction effects. Angle Orthod 2001;71:90-102.

Palmer AR, Strobeck C. Fluctuating asymmetry analyses revisited. In: Polak M, editor. Developmental instability: causes and consequences. Oxford: Oxford University Press, 2003, p 279-319.

Peavy DC Jr, Dendrick GS. The effects of tooth movement on the palatine rugae. J Prosthet Dent 1967;18:536-42.

Proffit WR, Fields HW Jr, Moray LJ. Prevalence of malocclusion and orthodontic treatment need in the United States: estimates from the NHANES III survey. Int J Adult Orthodon Orthognath Surg 1998;13:97-106.

Proffit W. Contemporary orthodontics, 3rd ed. St Louis: CV Mosby Company, 2000.

Saatci P, Yukay F. The effect of premolar extractions on tooth-size discrepancy. Am J Orthod Dentofacial Orthop 1997;111:428-34.

Schoppe RJ. An analysis of second premolar extraction procedures. Angle Orthod 1964;34:292-302.

Schwab DT. Extraction effects on the dental profile in borderline cases. Angle Orthod 1963;33:120-2. 
Sheppe JH. Six arch changes in first bicuspid extraction therapy in normal arches. Int J Orthod 1969;7:166-70.

Staggers JA. Vertical changes following first premolar extractions. Am J Orthod Dentofacial Orthop 1994;105:19-24.

Steyn CL, du Preez RJ, Harris AM. Differential premolar extractions. Am J Orthod Dentofacial Orthop 1997;112:480-6.

Tweed $\mathrm{CH}$. Indications for the extraction of teeth in orthodontic procedure. Am J Orthod Oral Surg 1944;30:405-28.

Vaden JL, Harris EF, Gardner RL. Relapse revisited. Am J Orthod Dentofacial Orthop 1997;111:543-53.

van der Linden FPGM. Changes in the position of posterior teeth in relation to ruga points. Am J Orthod 1978;74:142-61.

Van Valen L. A study of fluctuating asymmetry. Evolution 1962;16:125-42.

Williams R, Hosila FJ. The effect of different extraction sites upon incisor retraction. Am J Orthod 1976;69:388-410.

Woo TL. On the asymmetry of the human skull. Biometrika 1931;22:324-52.

Woo TL. A biometrical study of the human malar bone. Biometrika 1938;29:11323. 
APPENDIX 


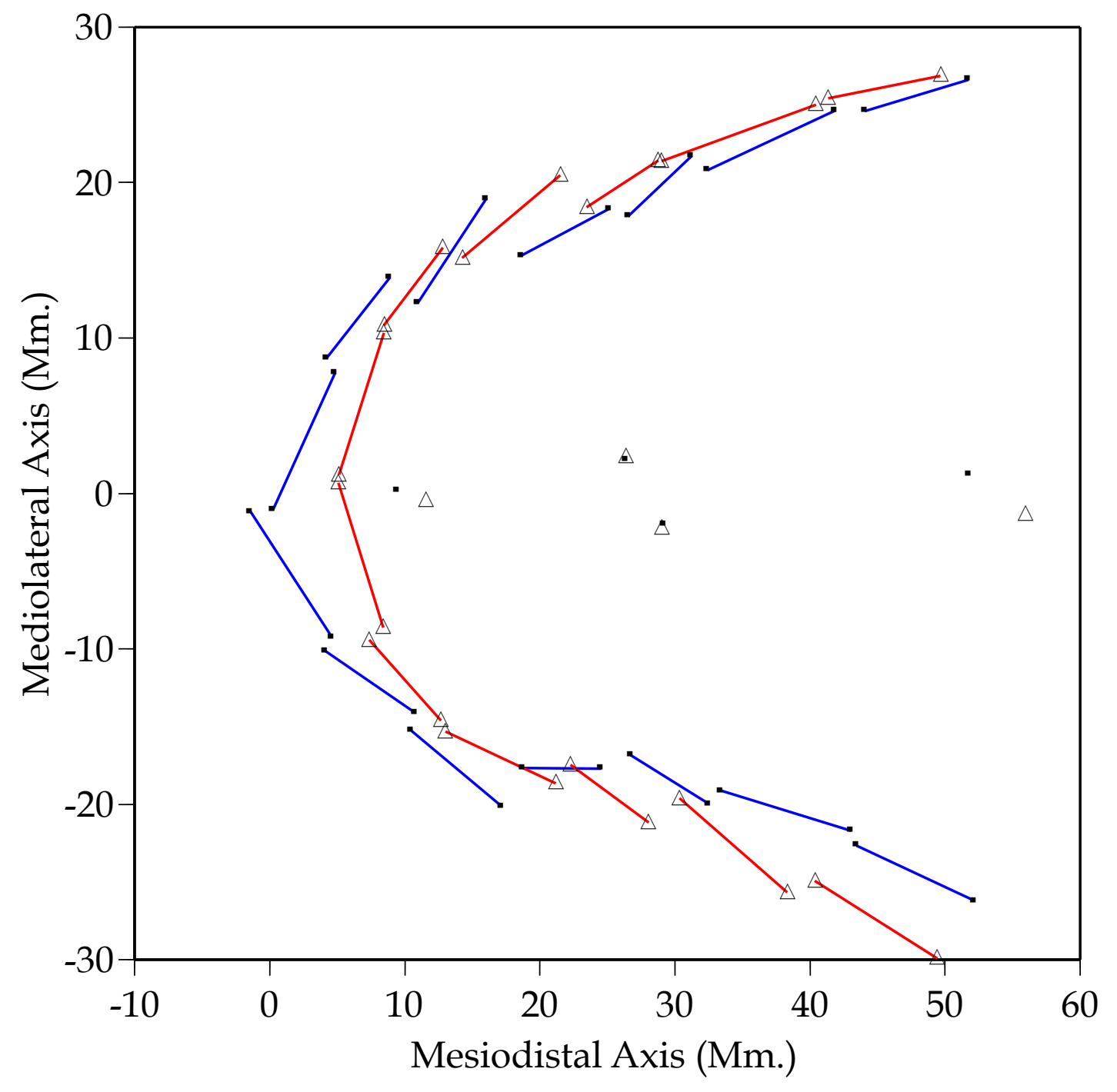

Fig. A-1. Schematic plot of the in-treatment changes in tooth position for case 1932. Registration is on the medial tips of the posterior palatal rugae. Symbols: dots are pretreatment; triangles are posttreatment. 


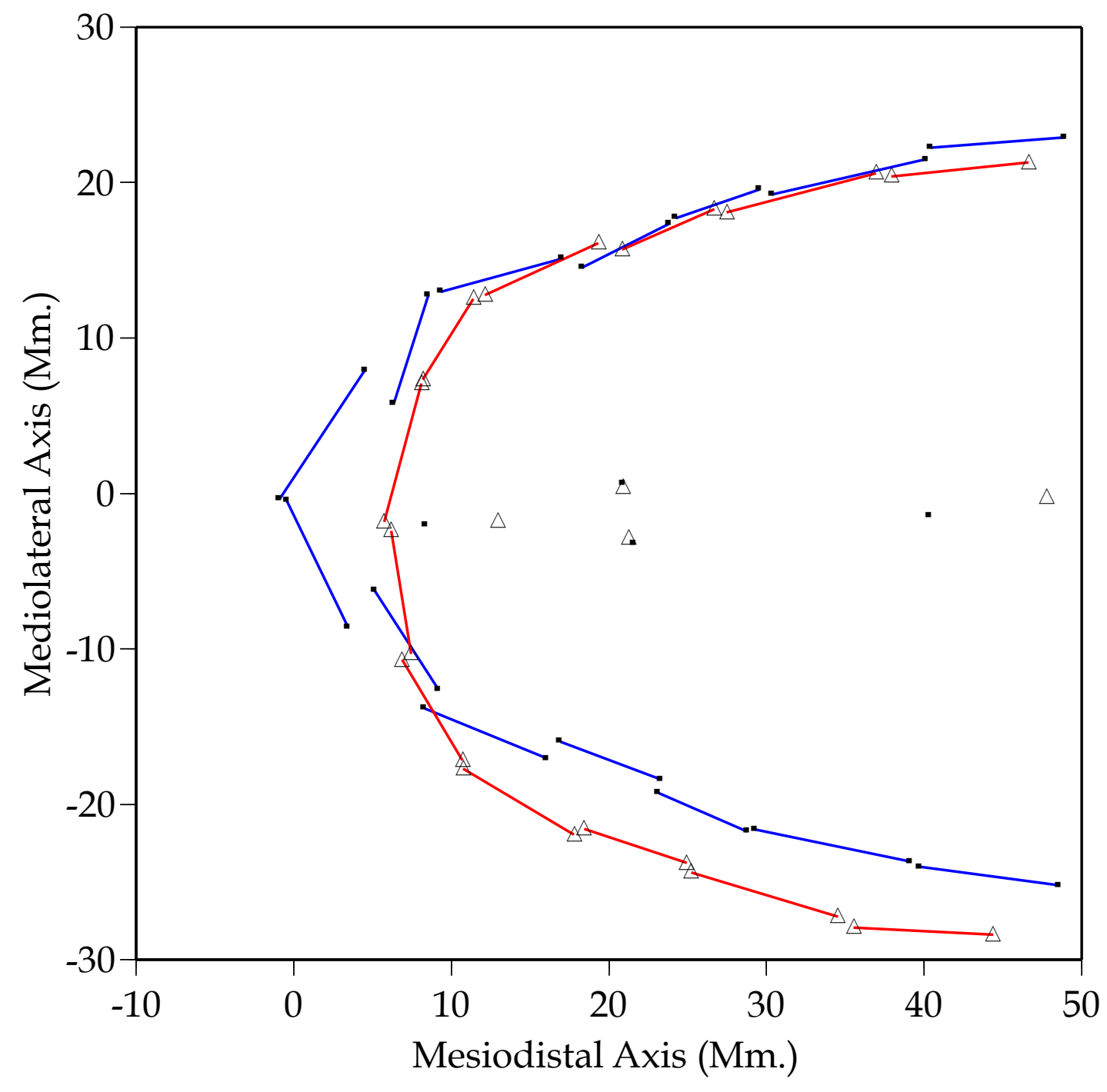

Fig. A-2. Schematic plot of the in-treatment changes in tooth position for case 20. Registration is on the medial tips of the posterior palatal rugae. Symbols: dots are pretreatment; triangles are posttreatment. 


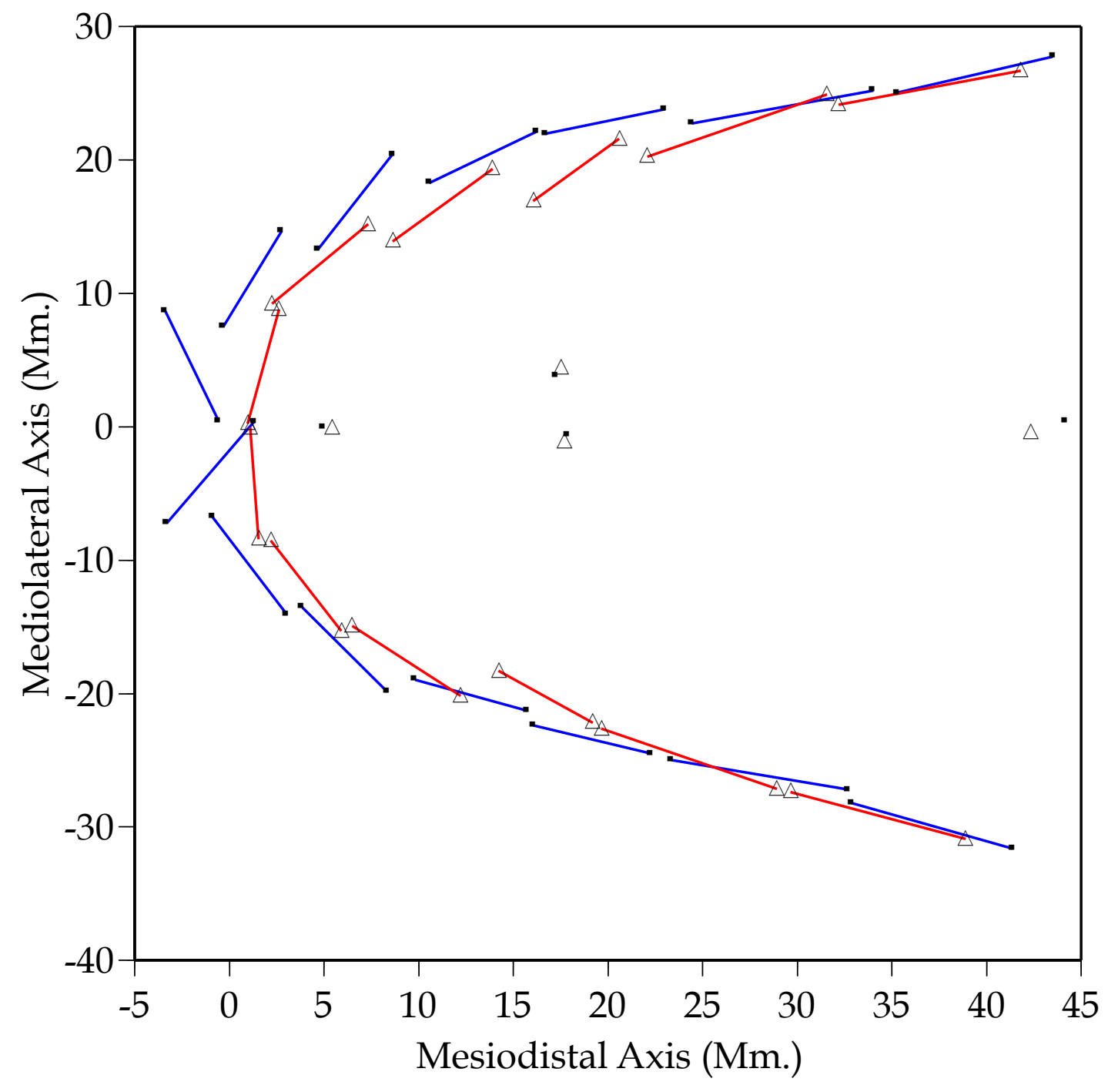

Fig. A-3. Schematic plot of the in-treatment changes in tooth position for case 58. Registration is on the medial tips of the posterior palatal rugae. Symbols: dots are pretreatment; triangles are posttreatment. 


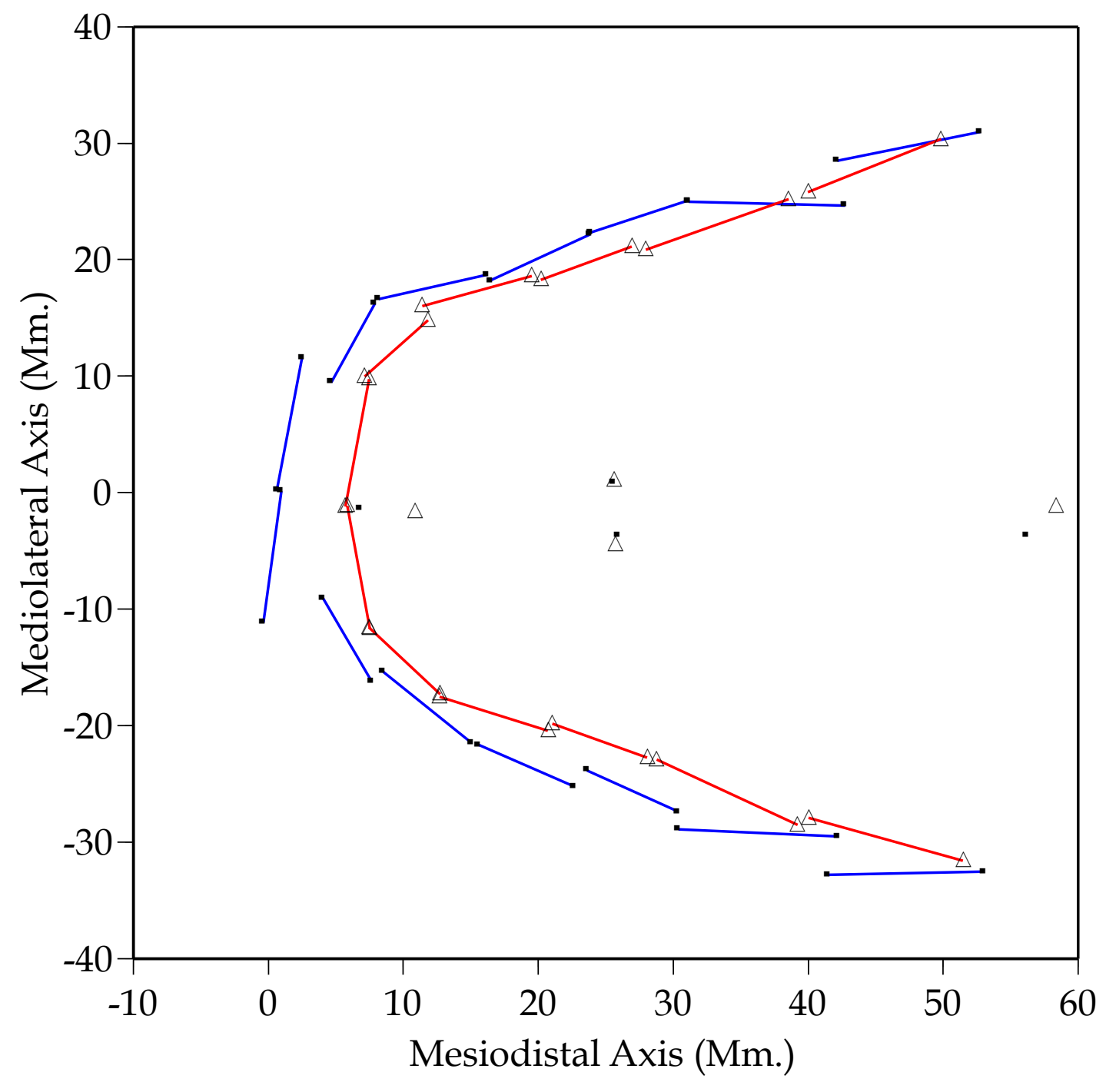

Fig. A-4. Schematic plot of the in-treatment changes in tooth position for case 1856. Registration is on the medial tips of the posterior palatal rugae. Symbols: dots are pretreatment; triangles are posttreatment. 


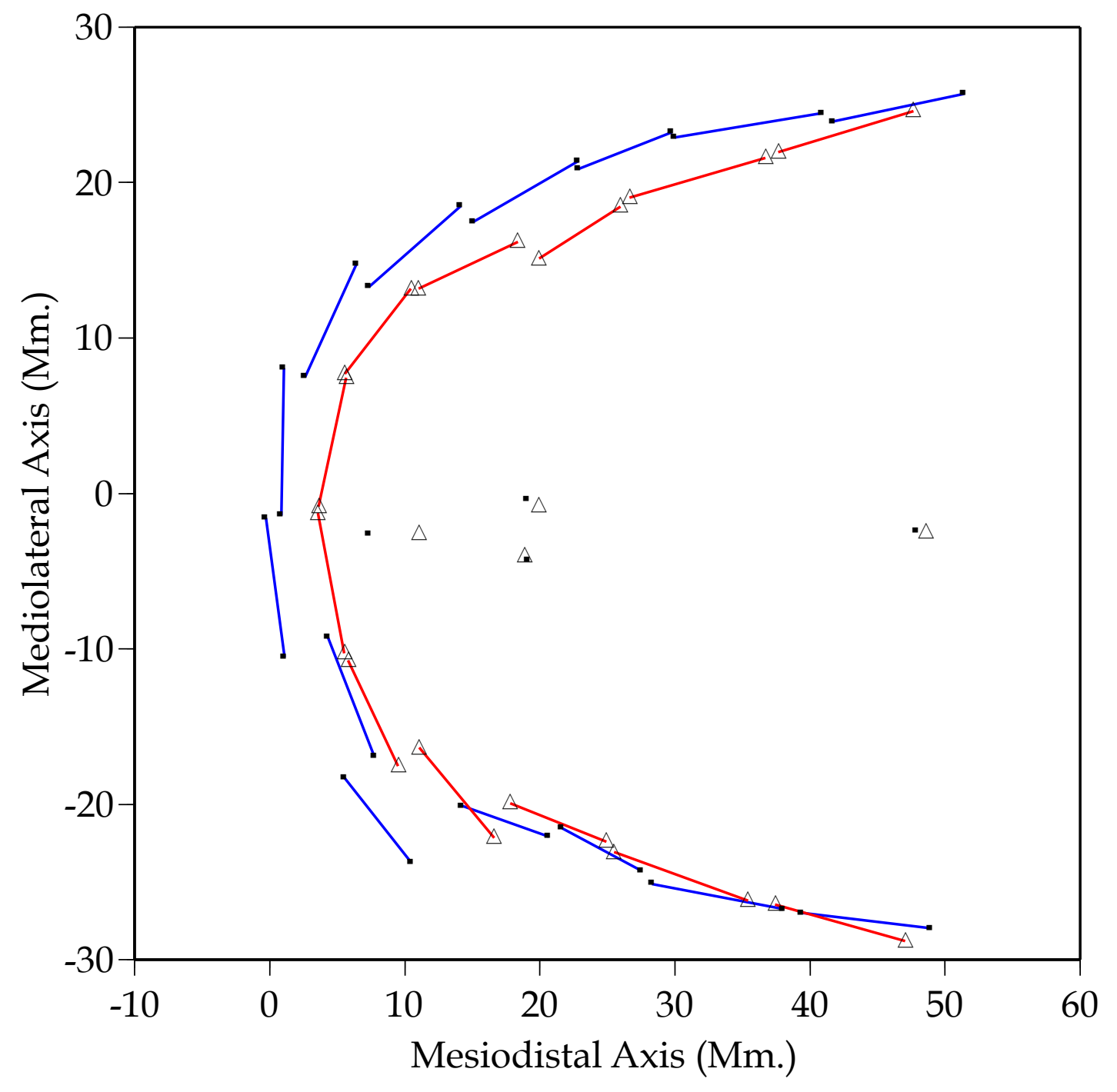

Fig. A-5. Schematic plot of the in-treatment changes in tooth position for case 1220. Registration is on the medial tips of the posterior palatal rugae. Symbols: dots are pretreatment; triangles are posttreatment. 


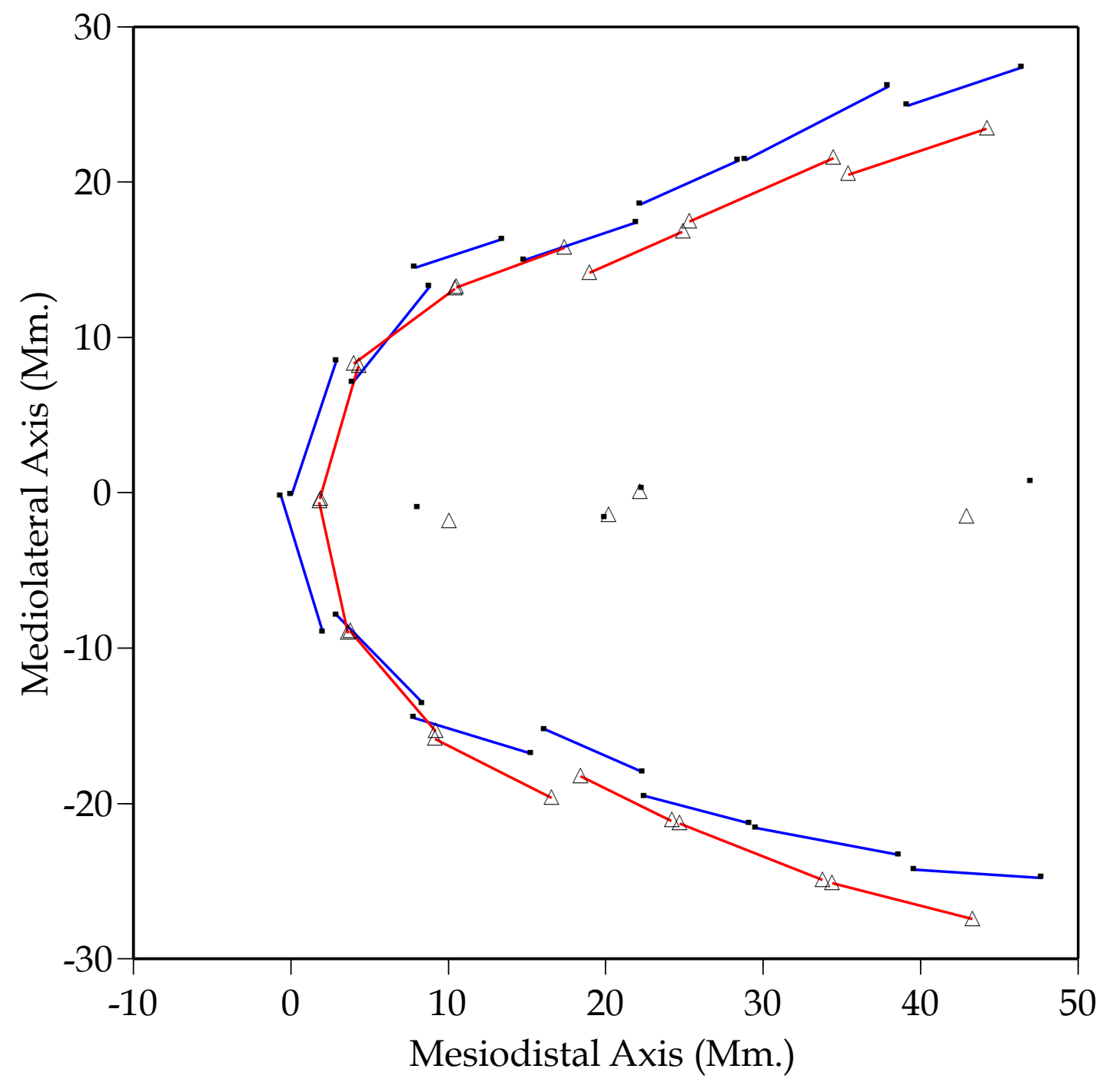

Fig. A-6. Schematic plot of the in-treatment changes in tooth position for case 918. Registration is on the medial tips of the posterior palatal rugae. Symbols: dots are pretreatment; triangles are posttreatment. 


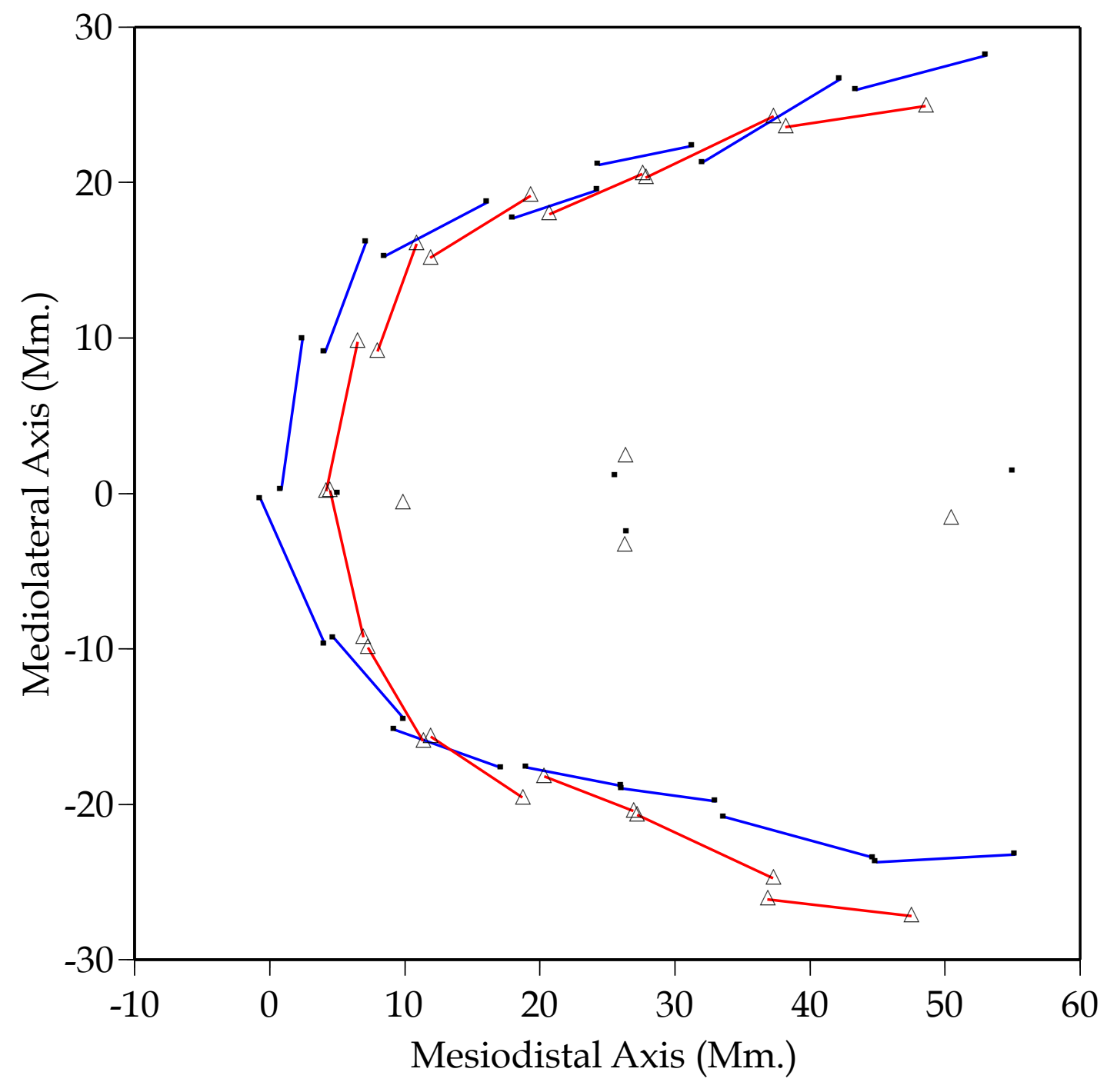

Fig. A-7. Schematic plot of the in-treatment changes in tooth position for case 2191. Registration is on the medial tips of the posterior palatal rugae. Symbols: dots are pretreatment; triangles are posttreatment. 


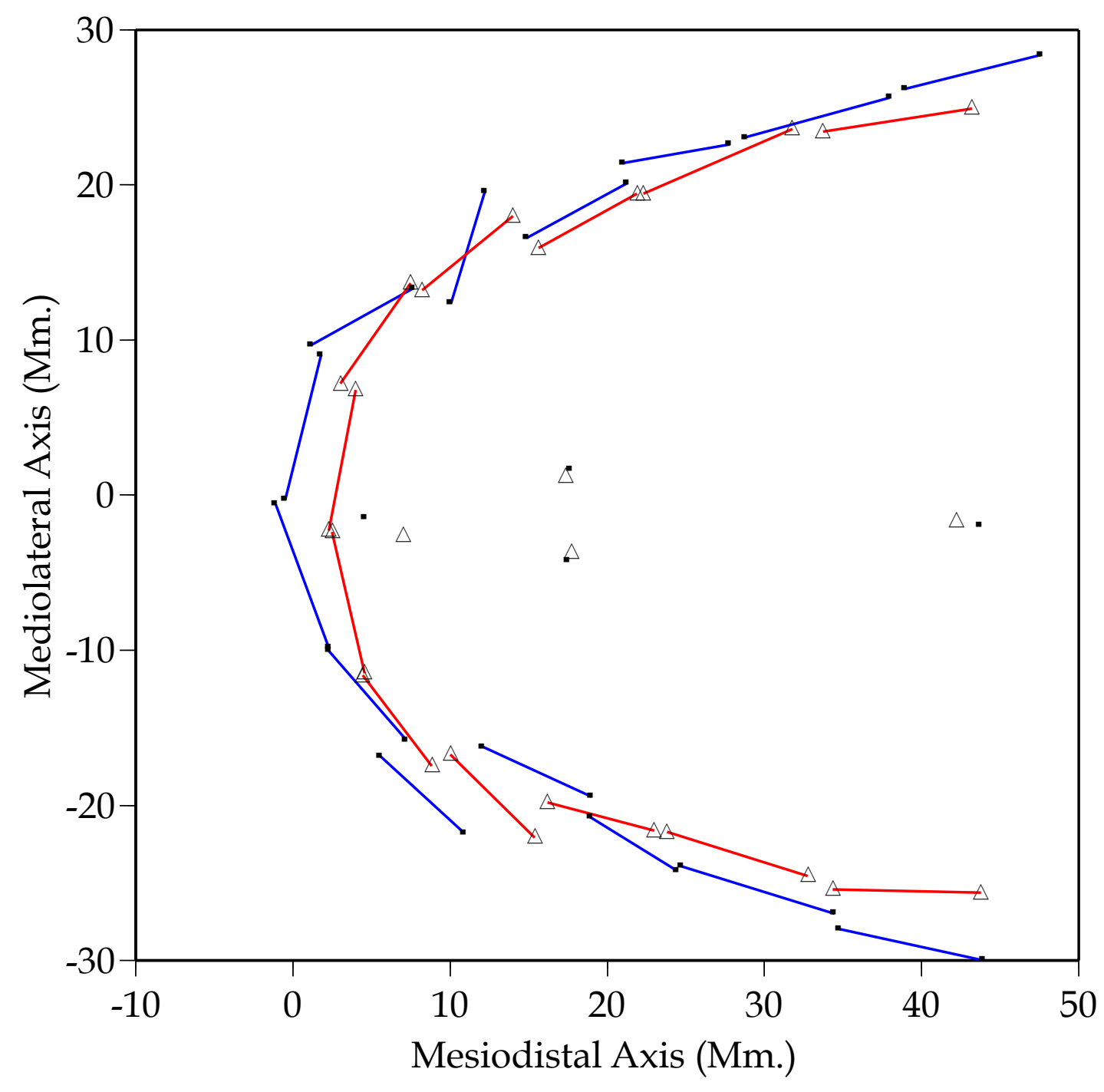

Fig. A-8. Schematic plot of the in-treatment changes in tooth position for case 627. Registration is on the medial tips of the posterior palatal rugae. Symbols: dots are pretreatment; triangles are posttreatment. 


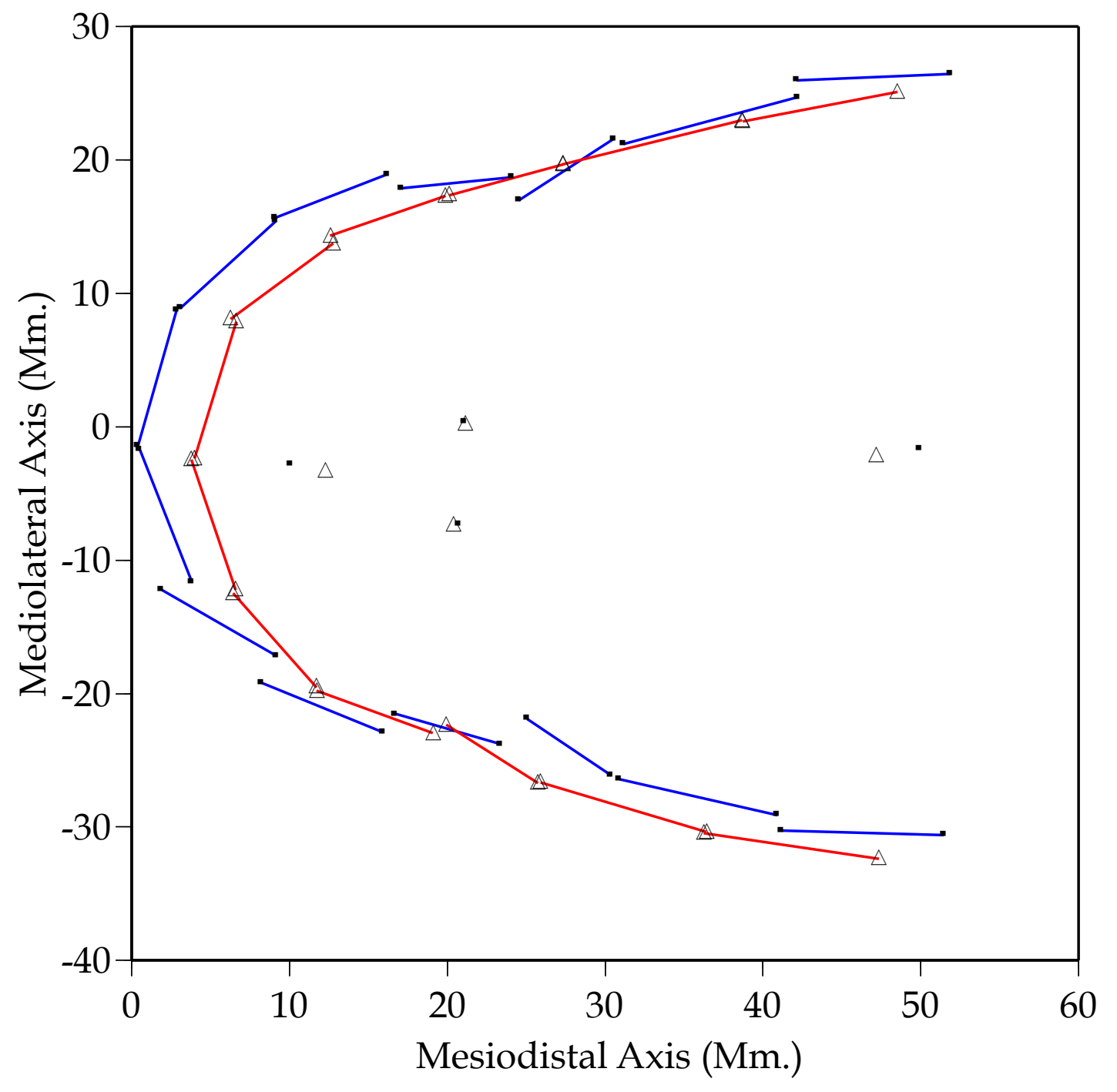

Fig. A-9. Schematic plot of the in-treatment changes in tooth position for case 743. Registration is on the medial tips of the posterior palatal rugae. Symbols: dots are pretreatment; triangles are posttreatment. 


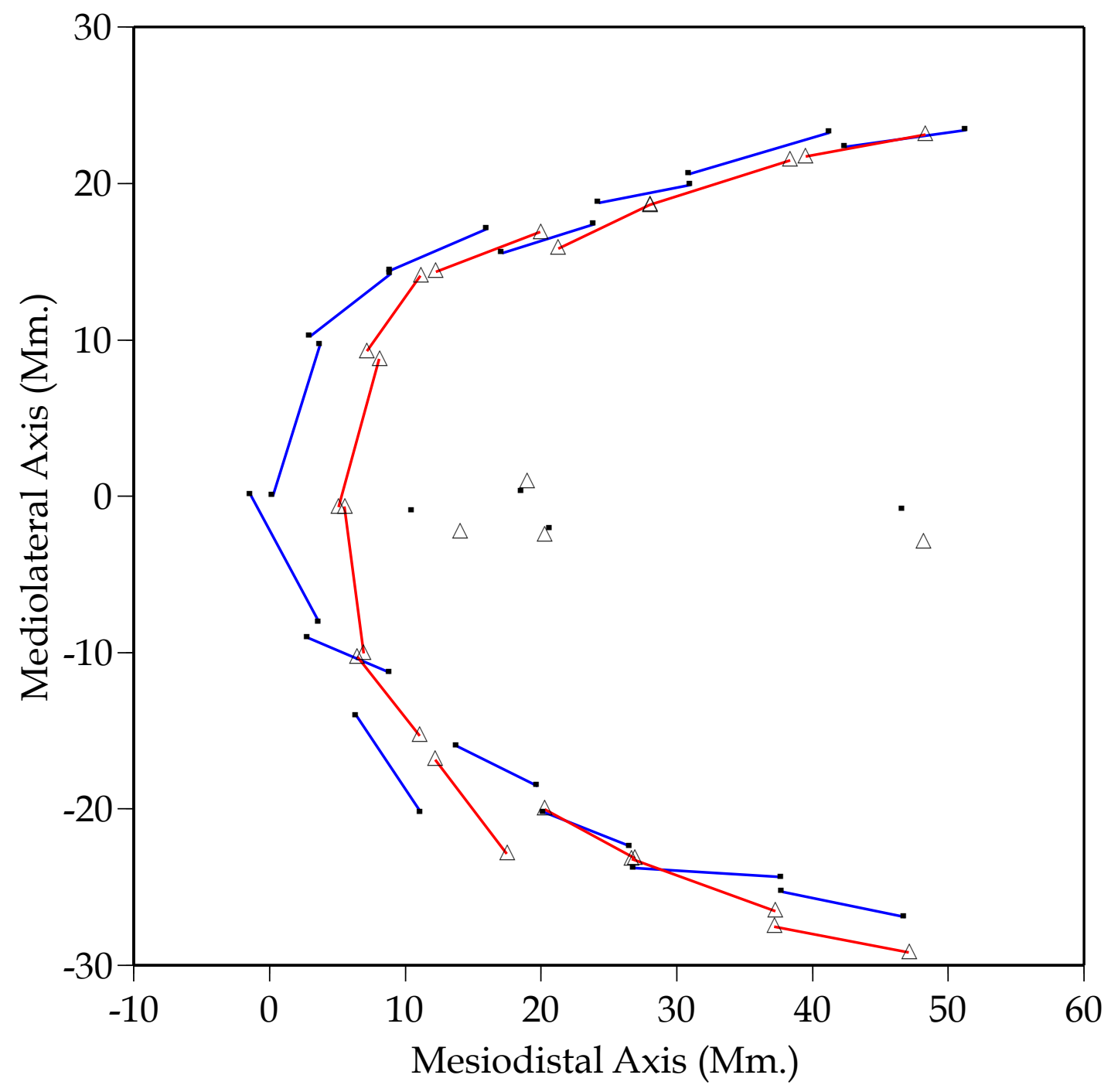

Fig. A-10. Schematic plot of the in-treatment changes in tooth position for case 280. Registration is on the medial tips of the posterior palatal rugae. Symbols: dots are pretreatment; triangles are posttreatment. 


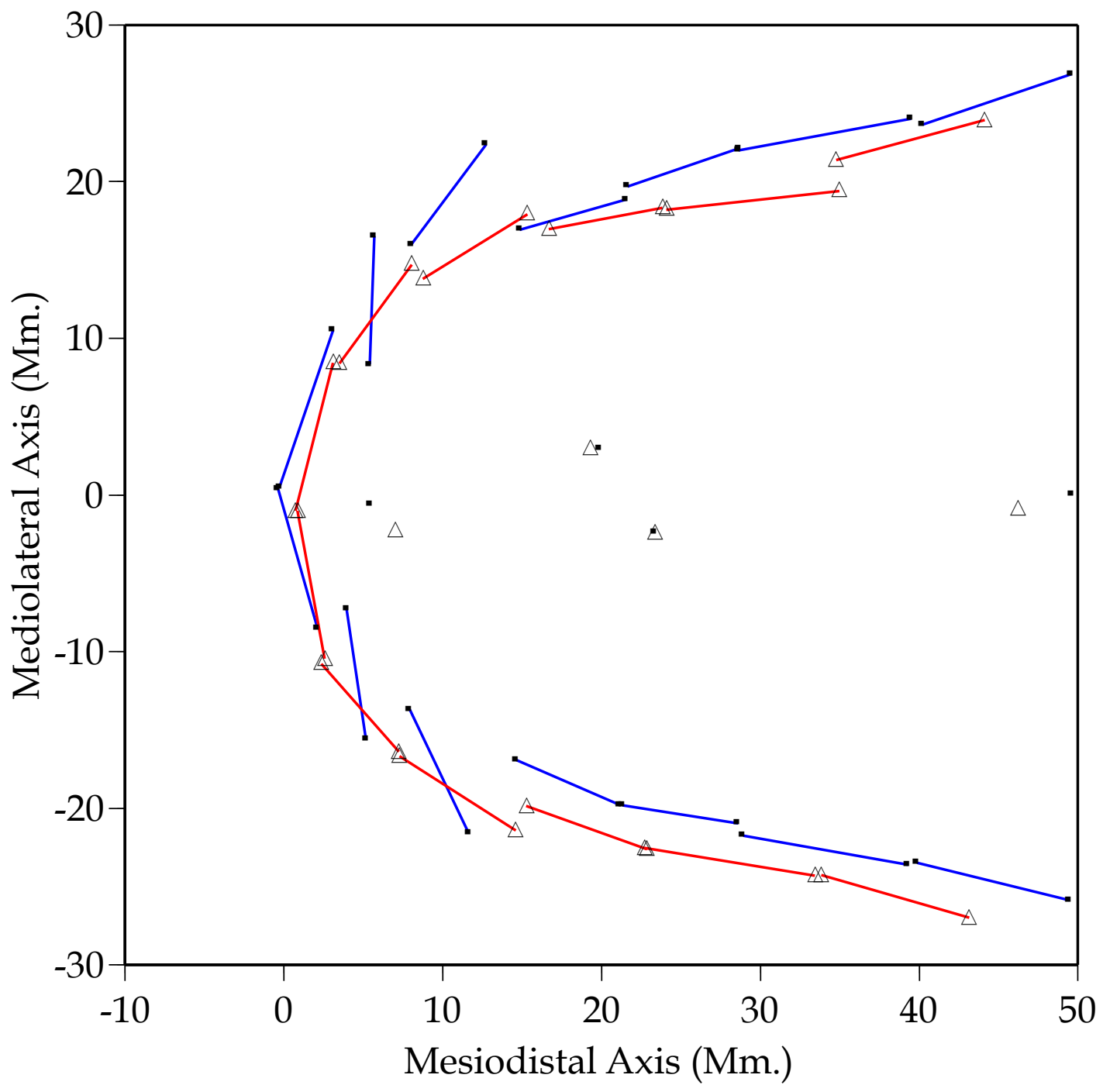

Fig. A-11. Schematic plot of the in-treatment changes in tooth position for case 1831. Registration is on the medial tips of the posterior palatal rugae. Symbols: dots are pretreatment; triangles are posttreatment. 


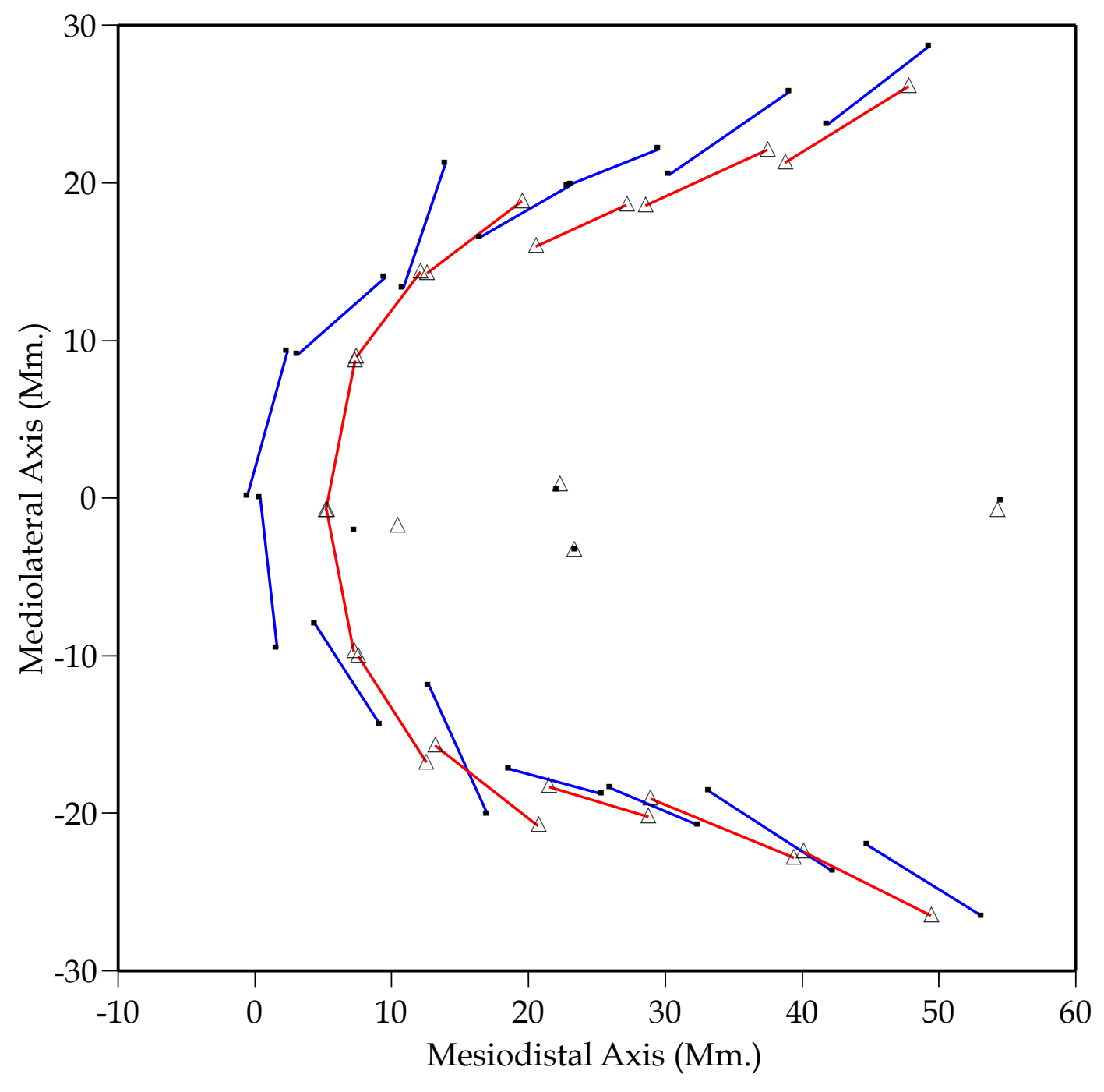

Fig. A-12. Schematic plot of the in-treatment changes in tooth position for case 1824. Registration is on the medial tips of the posterior palatal rugae. Symbols: dots are pretreatment; triangles are posttreatment. 


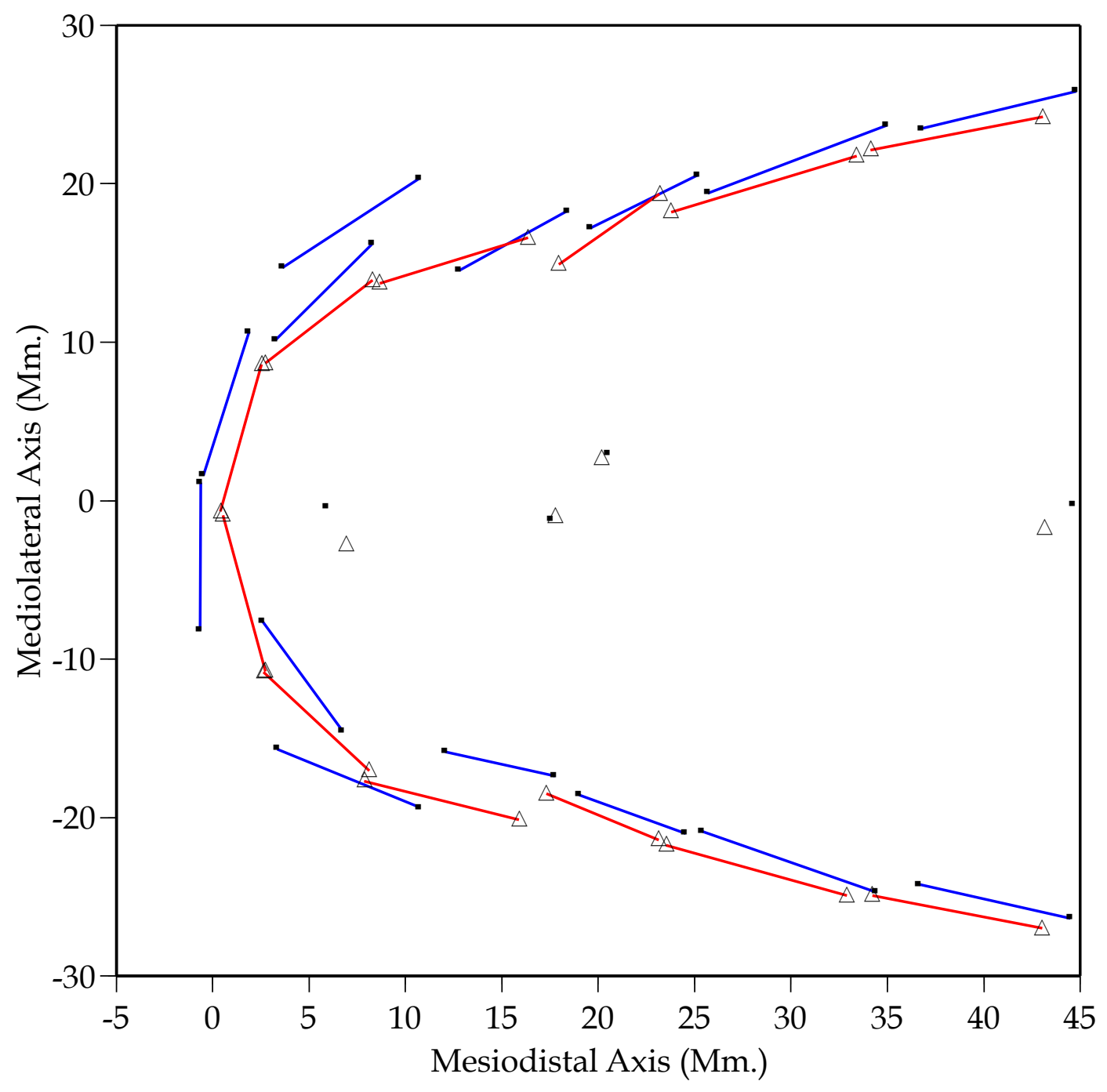

Fig. A-13. Schematic plot of the in-treatment changes in tooth position for case 775 . Registration is on the medial tips of the posterior palatal rugae. Symbols: dots are pretreatment; triangles are posttreatment. 


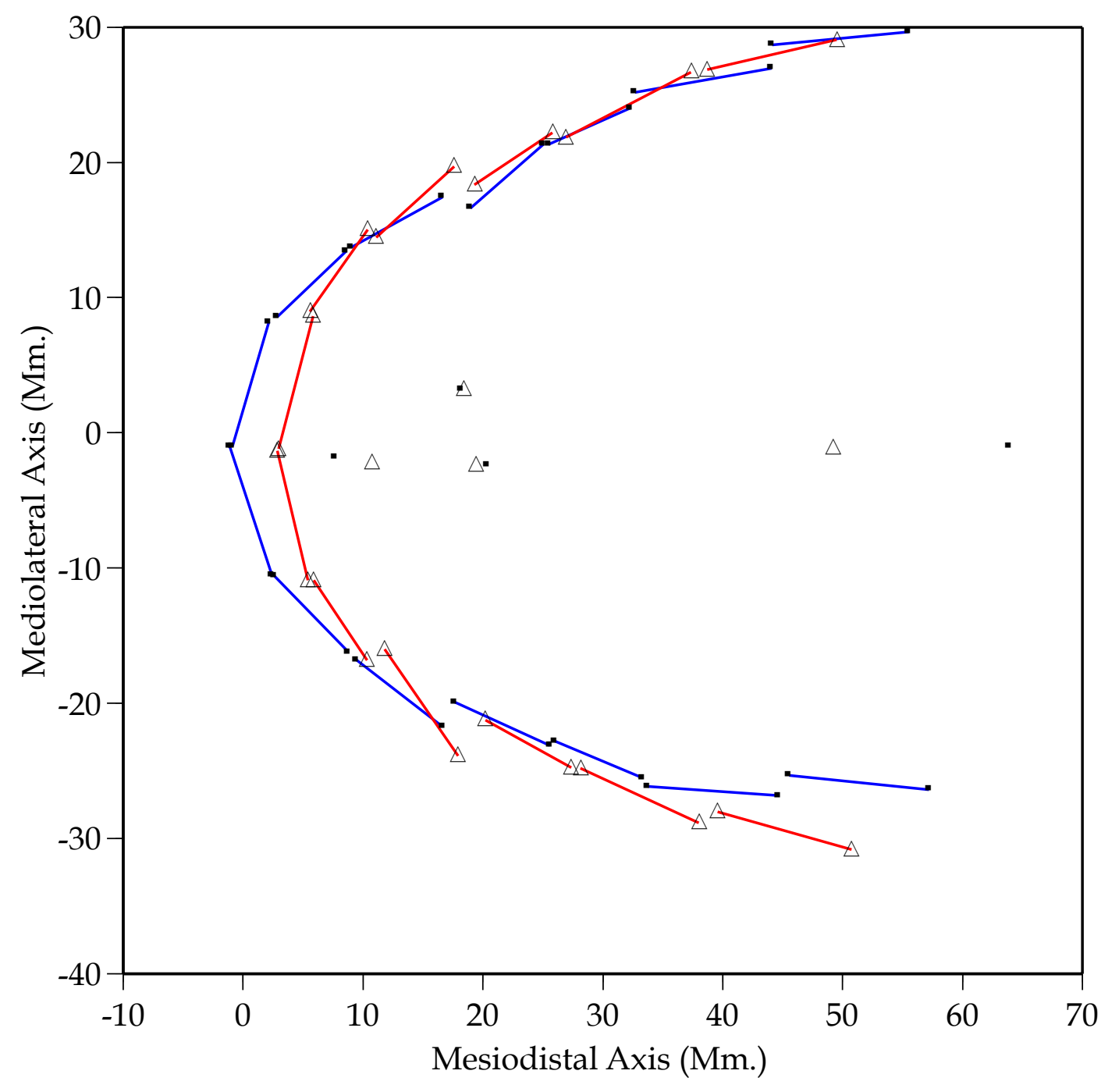

Fig. A-14. Schematic plot of the in-treatment changes in tooth position for case 301. Registration is on the medial tips of the posterior palatal rugae. Symbols: dots are pretreatment; triangles are posttreatment. 


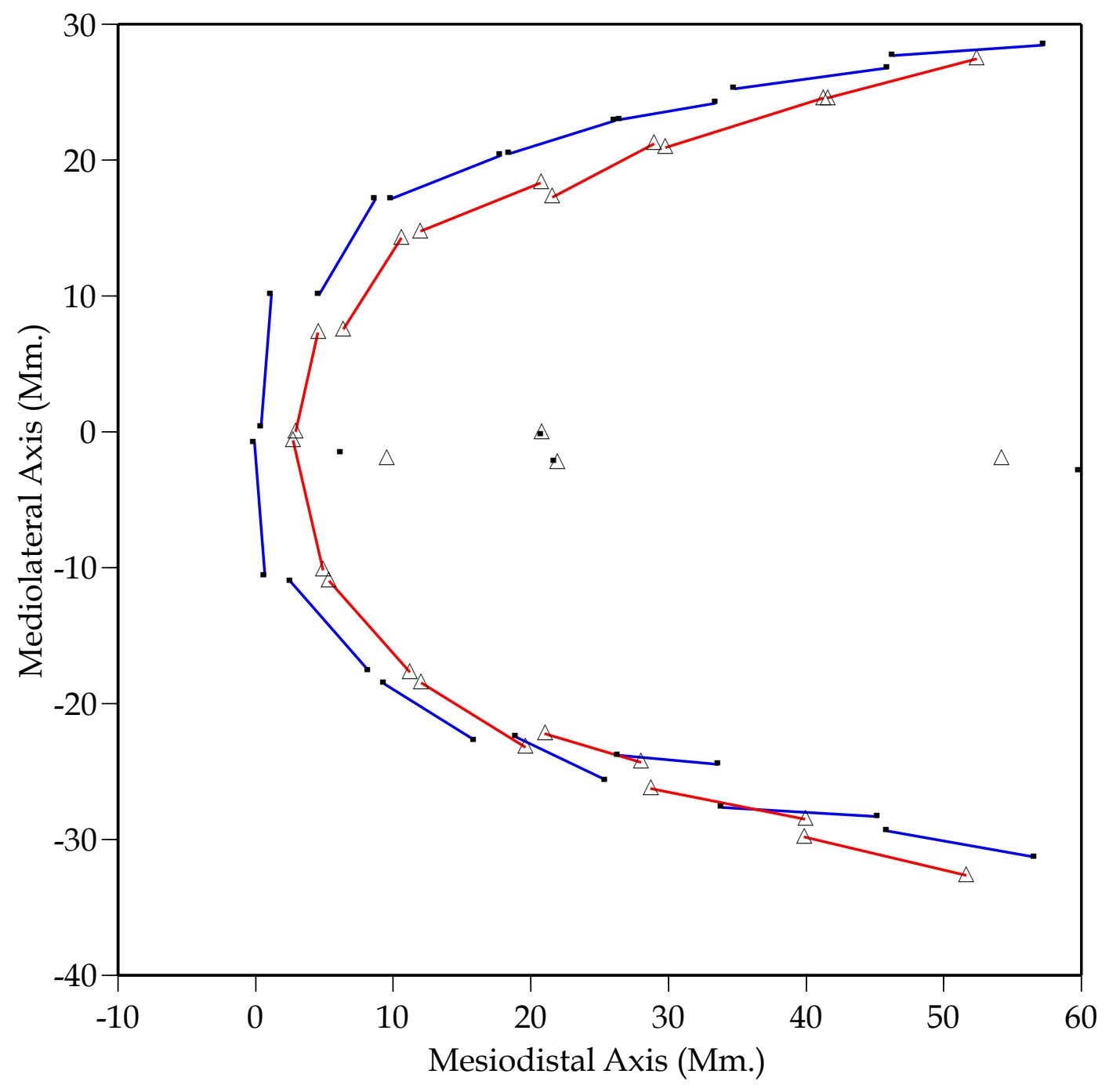

Fig. A-15. Schematic plot of the in-treatment changes in tooth position for case 2358. Registration is on the medial tips of the posterior palatal rugae. Symbols: dots are pretreatment; triangles are posttreatment. 


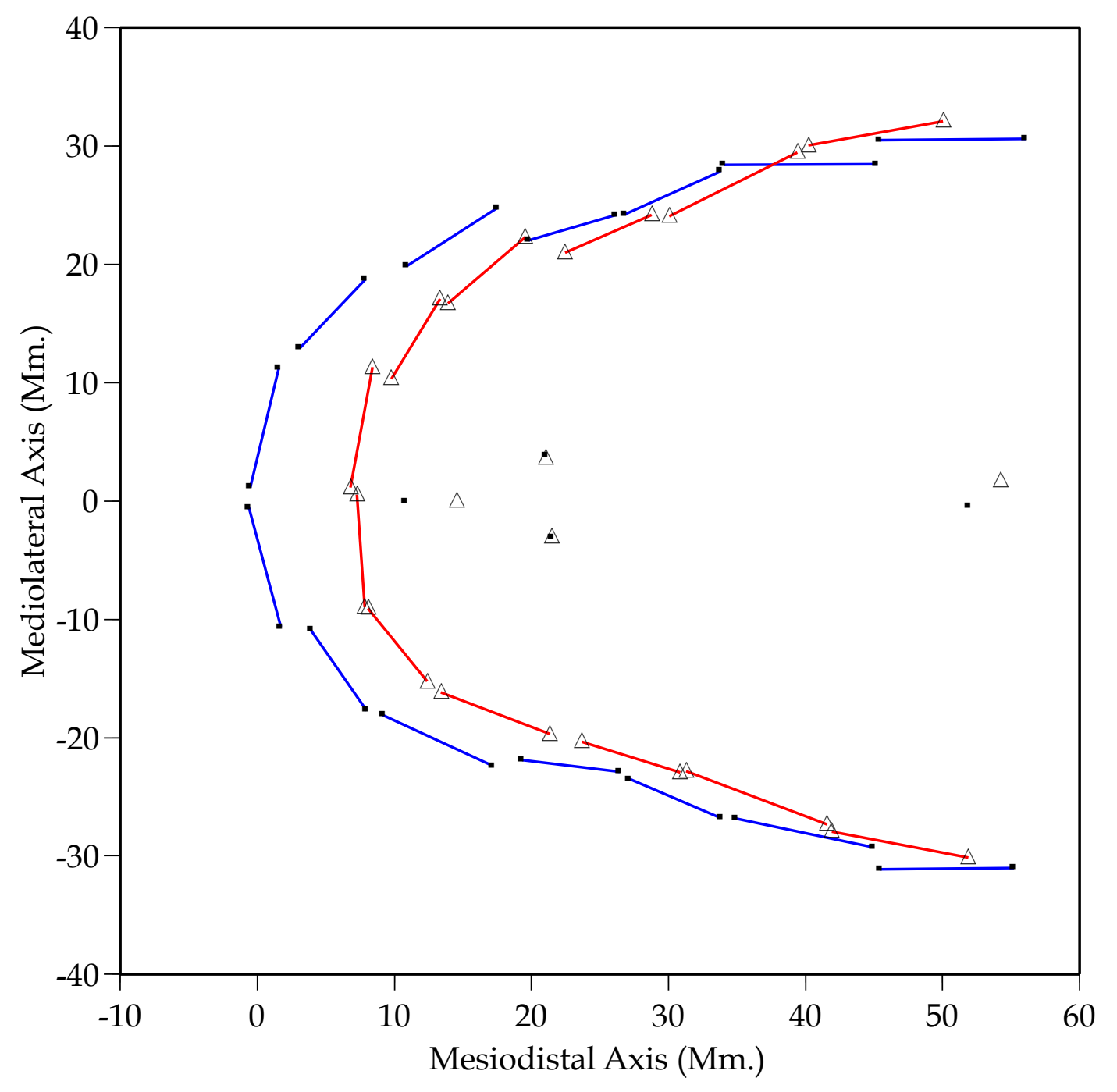

Fig. A-16. Schematic plot of the in-treatment changes in tooth position for case 25. Registration is on the medial tips of the posterior palatal rugae. Symbols: dots are pretreatment; triangles are posttreatment. 


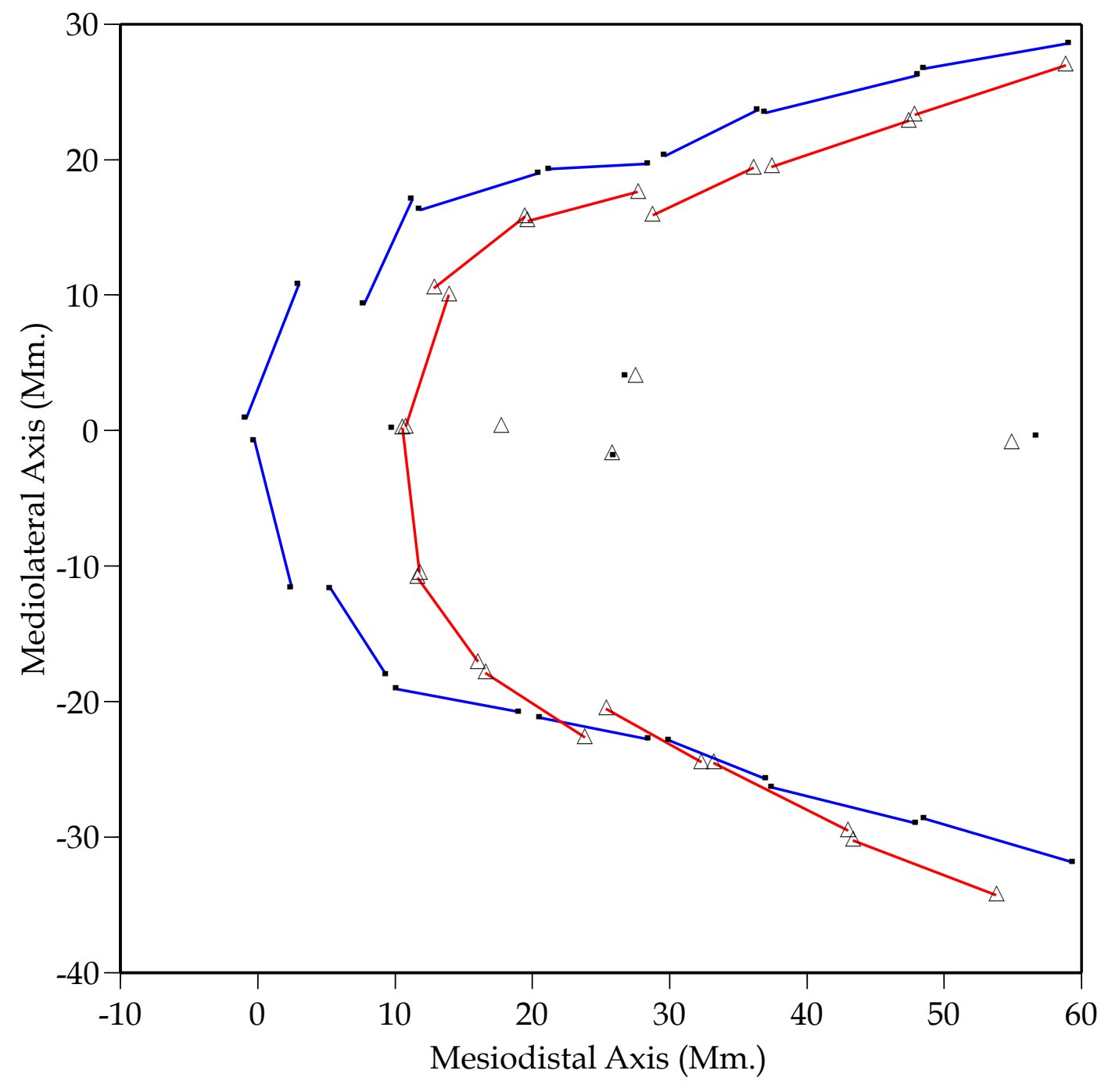

Fig. A-17. Schematic plot of the in-treatment changes in tooth position for case 427. Registration is on the medial tips of the posterior palatal rugae. Symbols: dots are pretreatment; triangles are posttreatment. 


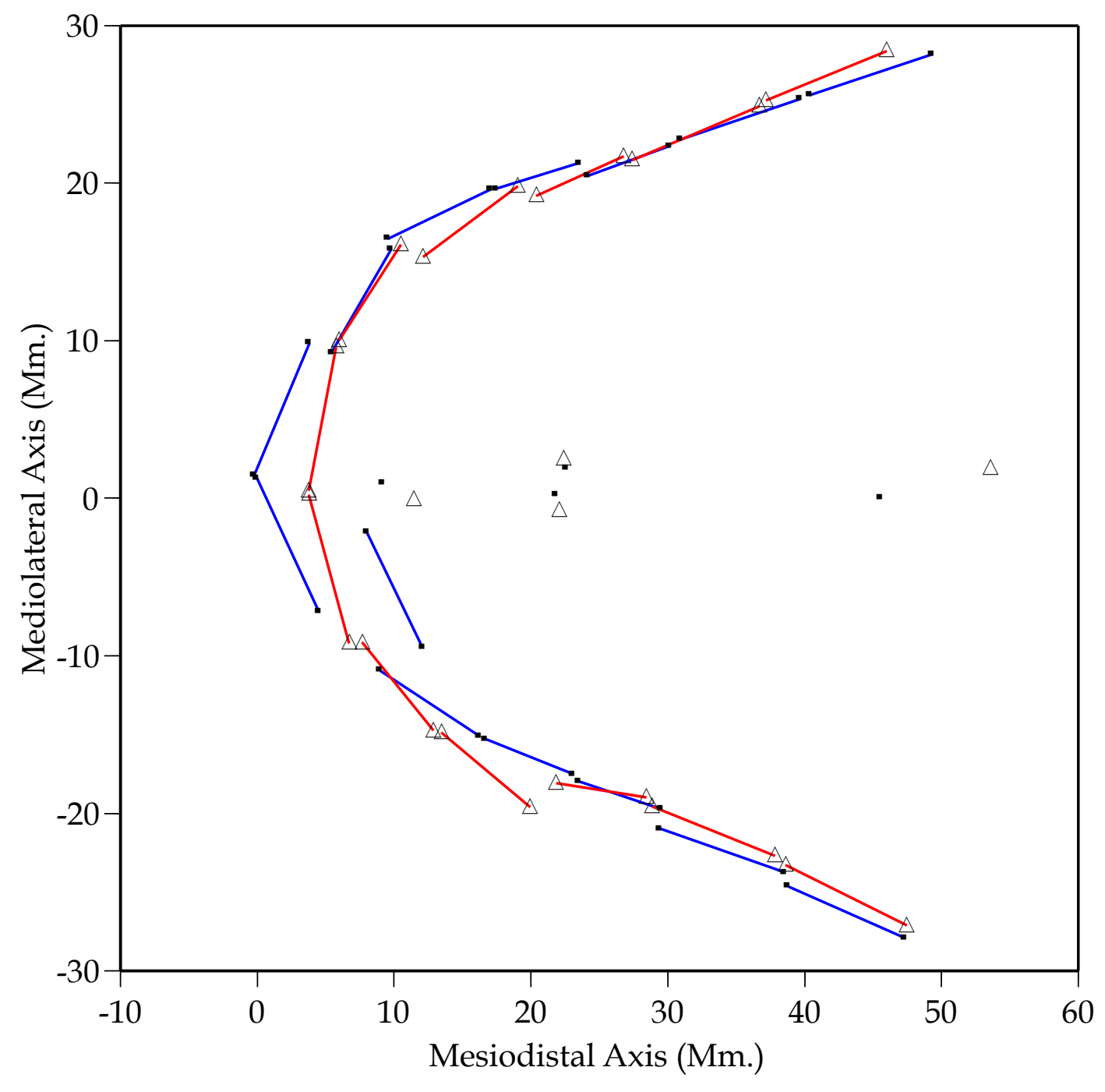

Fig. A-18. Schematic plot of the in-treatment changes in tooth position for case 73. Registration is on the medial tips of the posterior palatal rugae. Symbols: dots are pretreatment; triangles are posttreatment. 


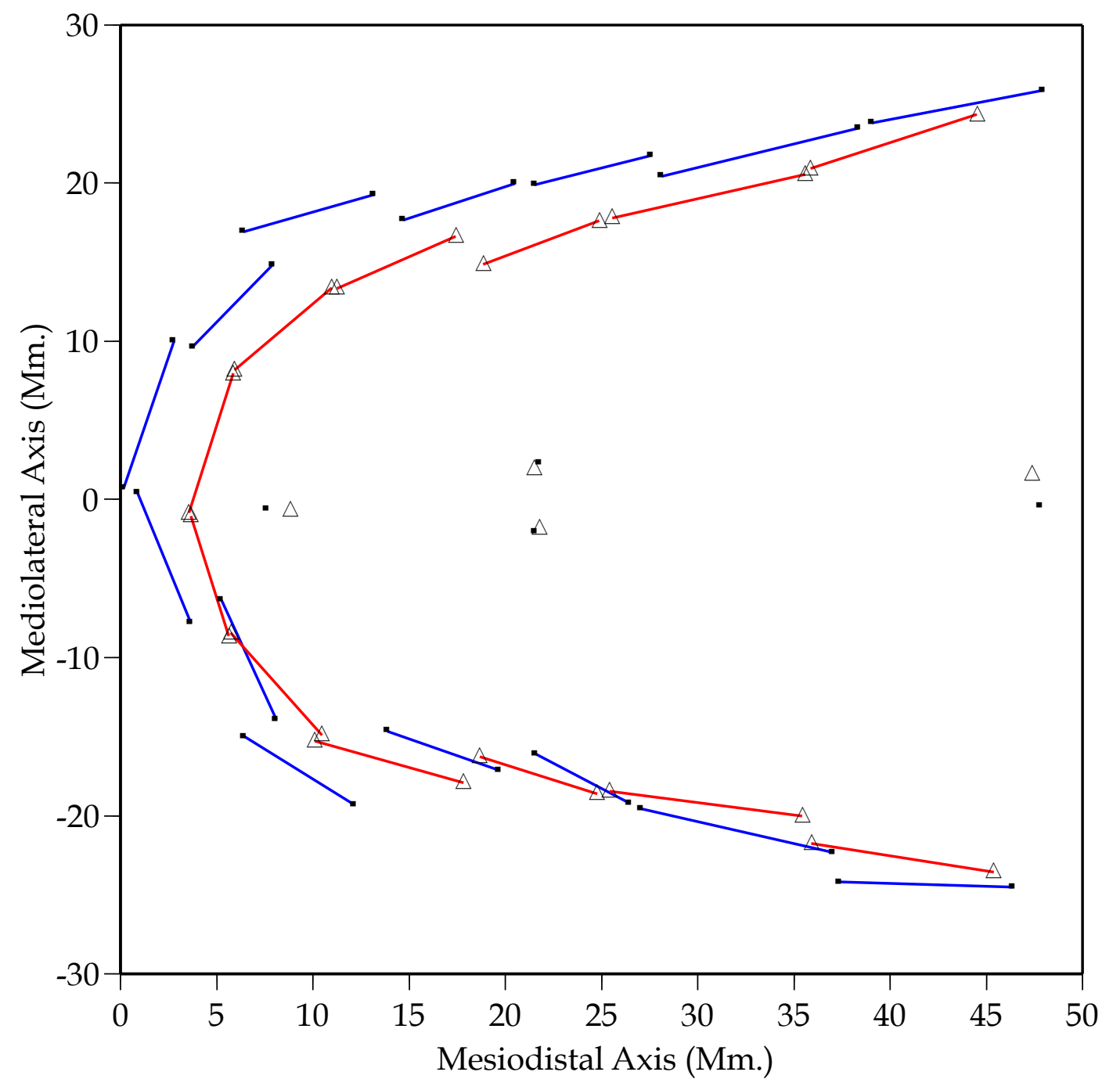

Fig. A-19. Schematic plot of the in-treatment changes in tooth position for case 204. Registration is on the medial tips of the posterior palatal rugae. Symbols: dots are pretreatment; triangles are posttreatment. 


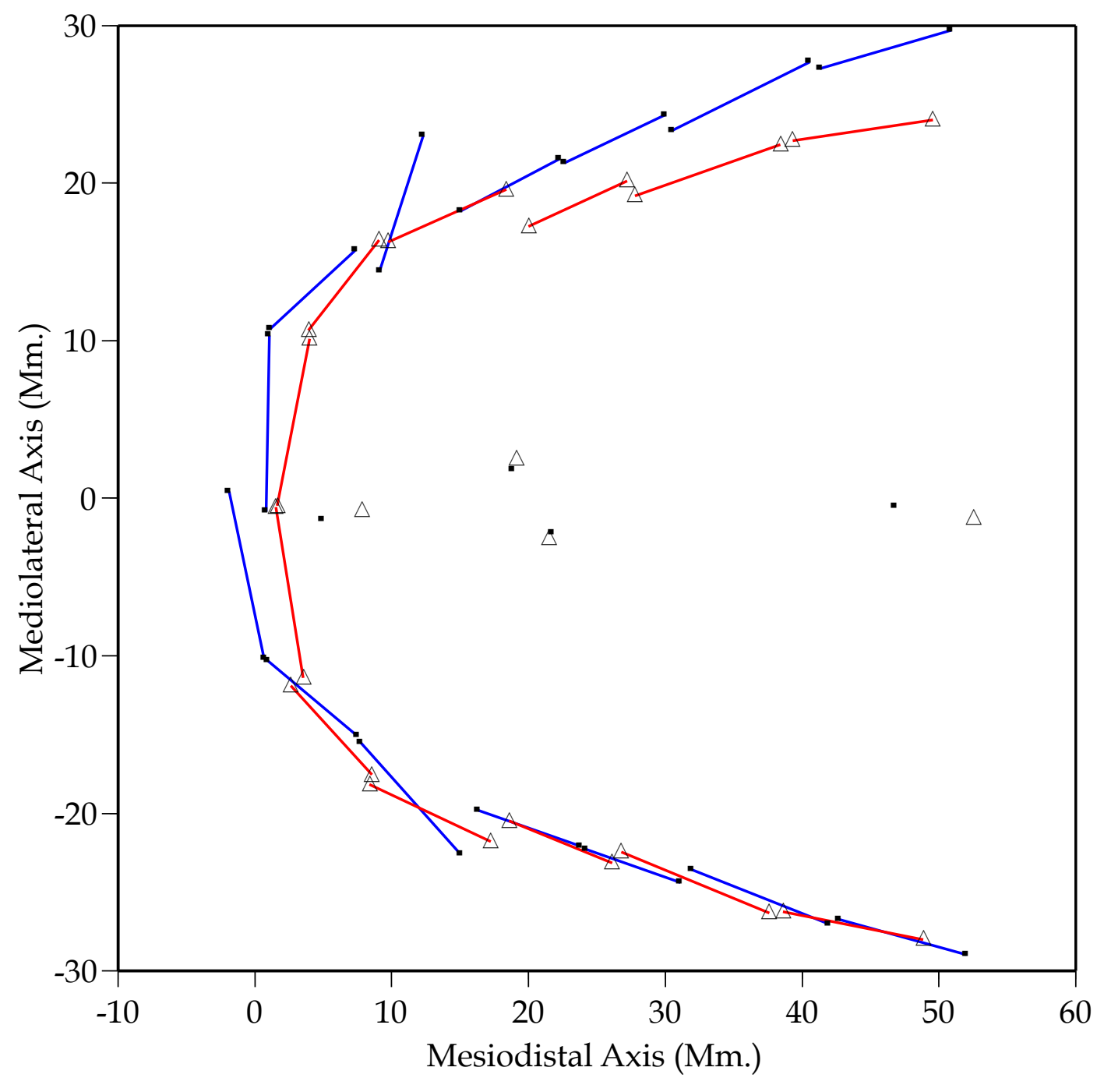

Fig. A-20. Schematic plot of the in-treatment changes in tooth position for case 1347. Registration is on the medial tips of the posterior palatal rugae. Symbols: dots are pretreatment; triangles are posttreatment. 


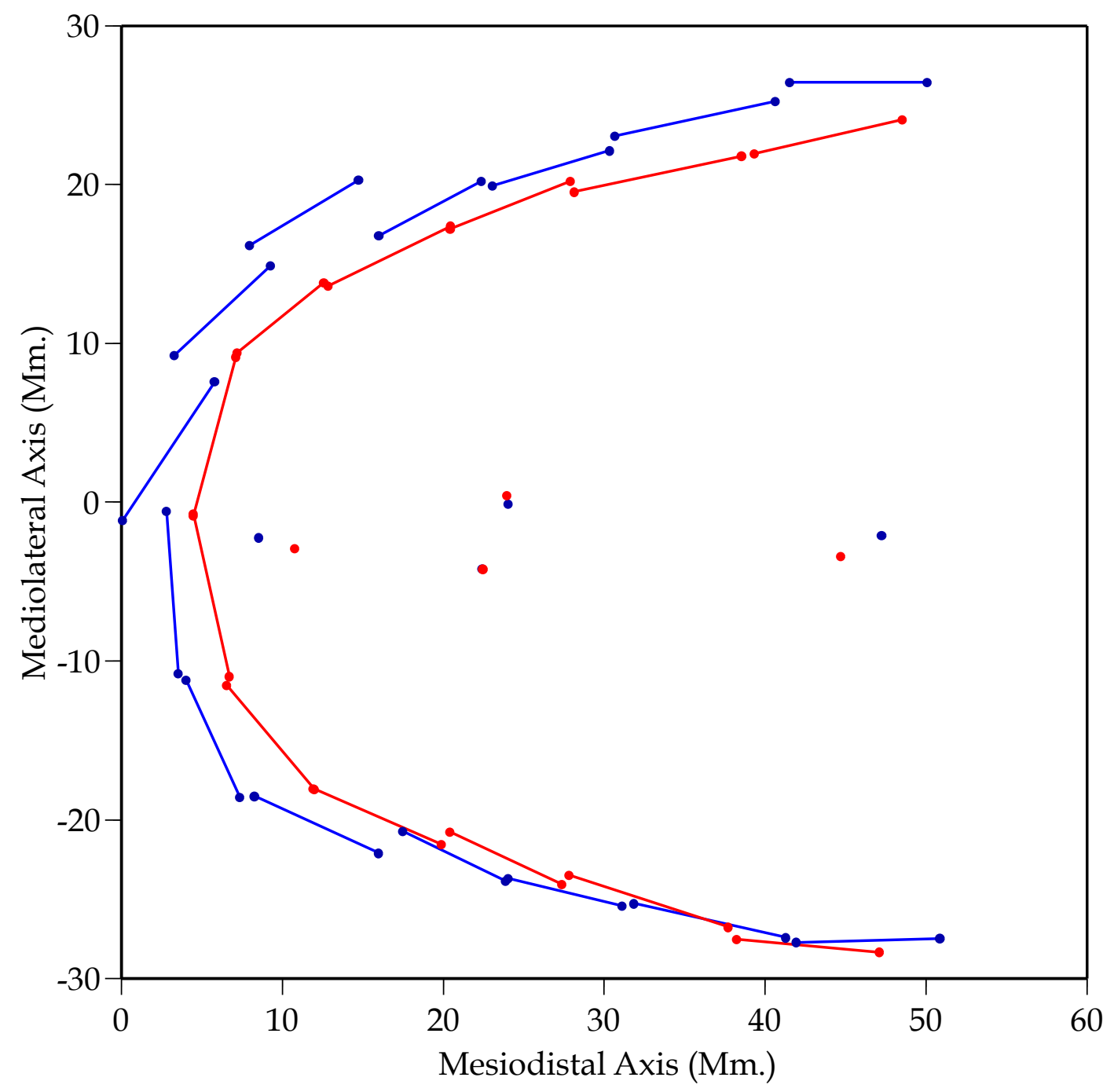

Fig. A-21. Schematic plot of the in-treatment changes in tooth position for case 659. Registration is on the medial tips of the posterior palatal rugae. Symbols: dots are pretreatment; triangles are posttreatment. 


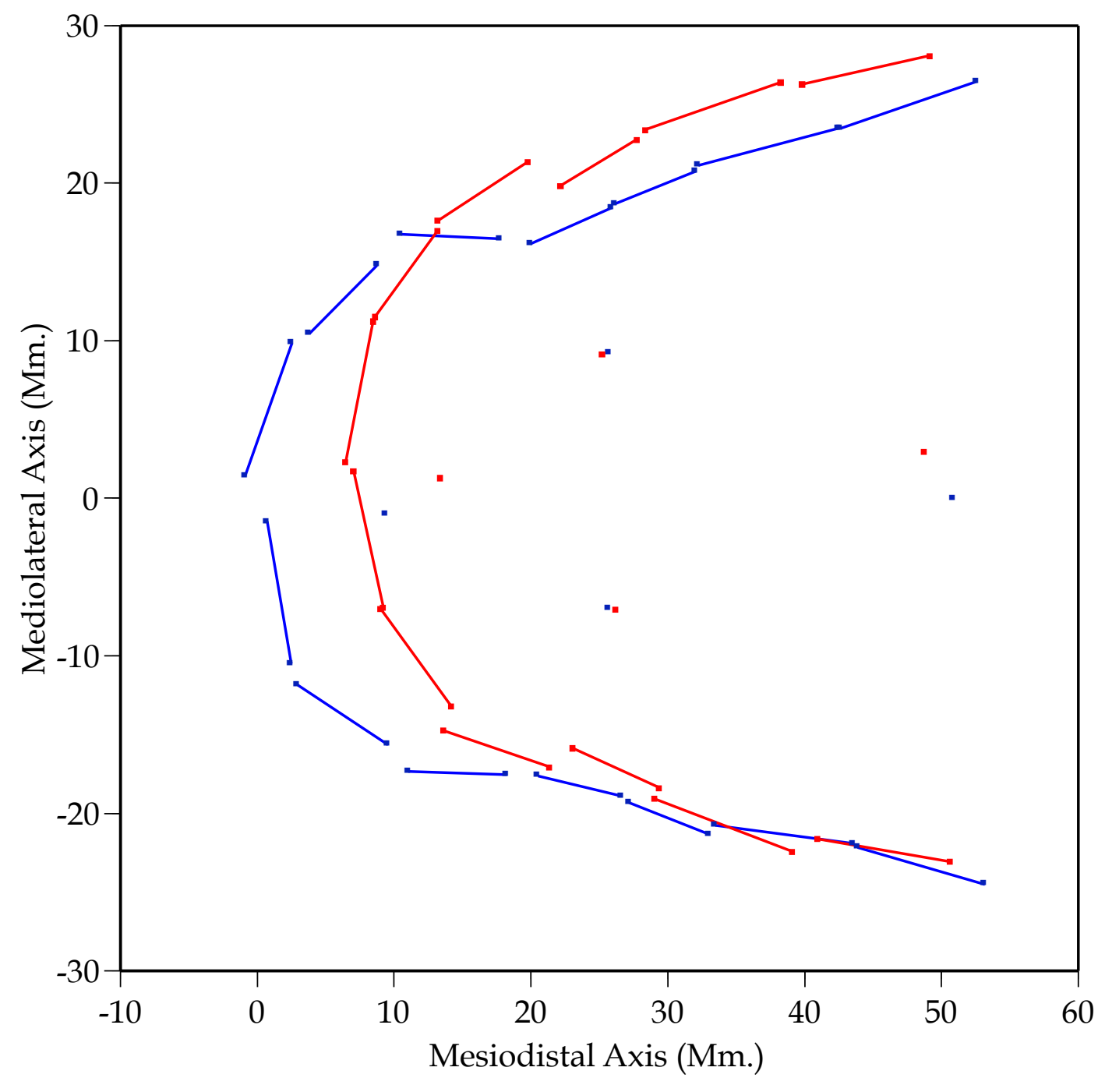

Fig. A-22. Schematic plot of the in-treatment changes in tooth position for case 226. Registration is on the medial tips of the posterior palatal rugae. Symbols: dots are pretreatment; triangles are posttreatment. 


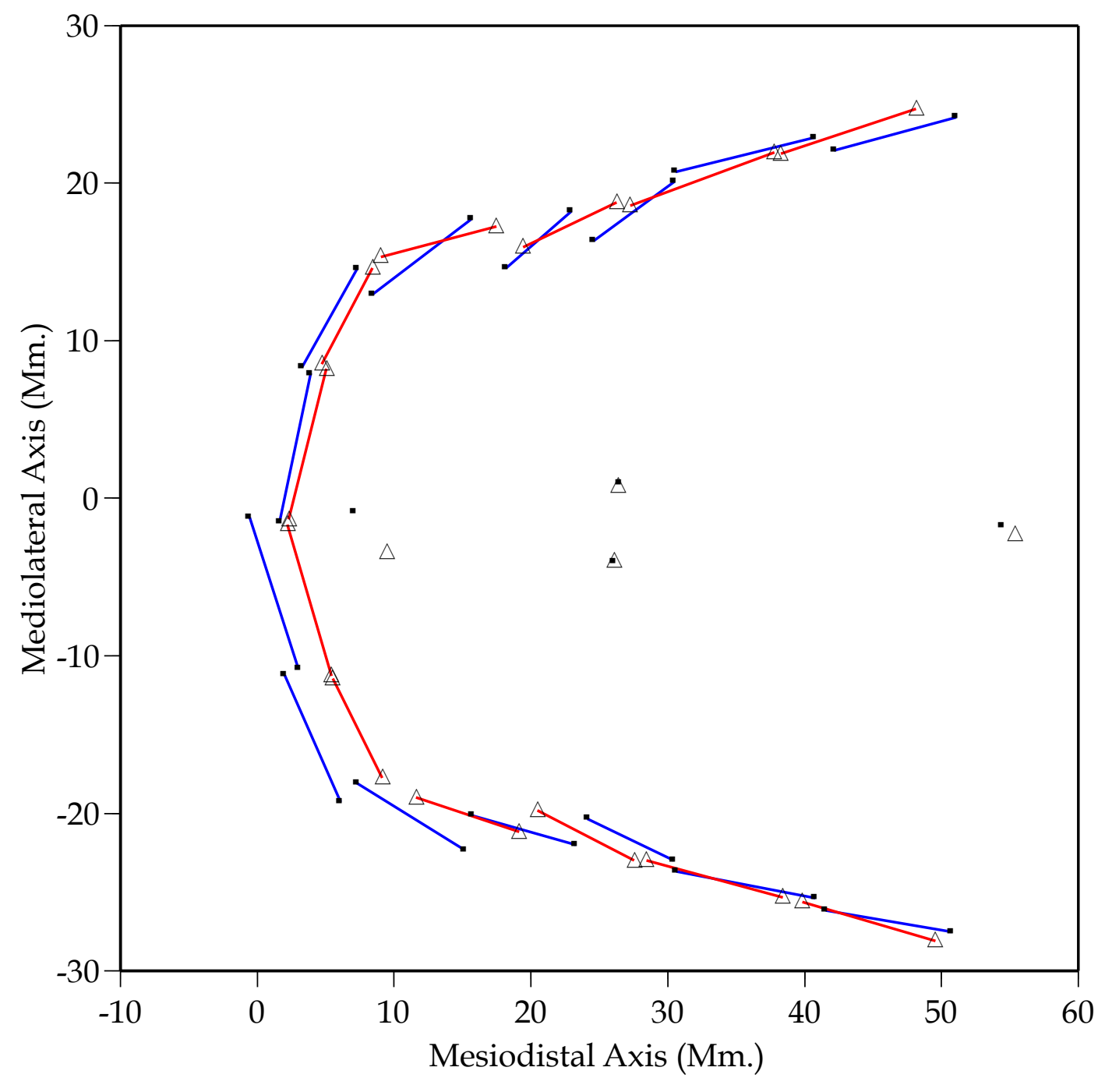

Fig. A-23. Schematic plot of the in-treatment changes in tooth position for case 2310. Registration is on the medial tips of the posterior palatal rugae. Symbols: dots are pretreatment; triangles are posttreatment. 


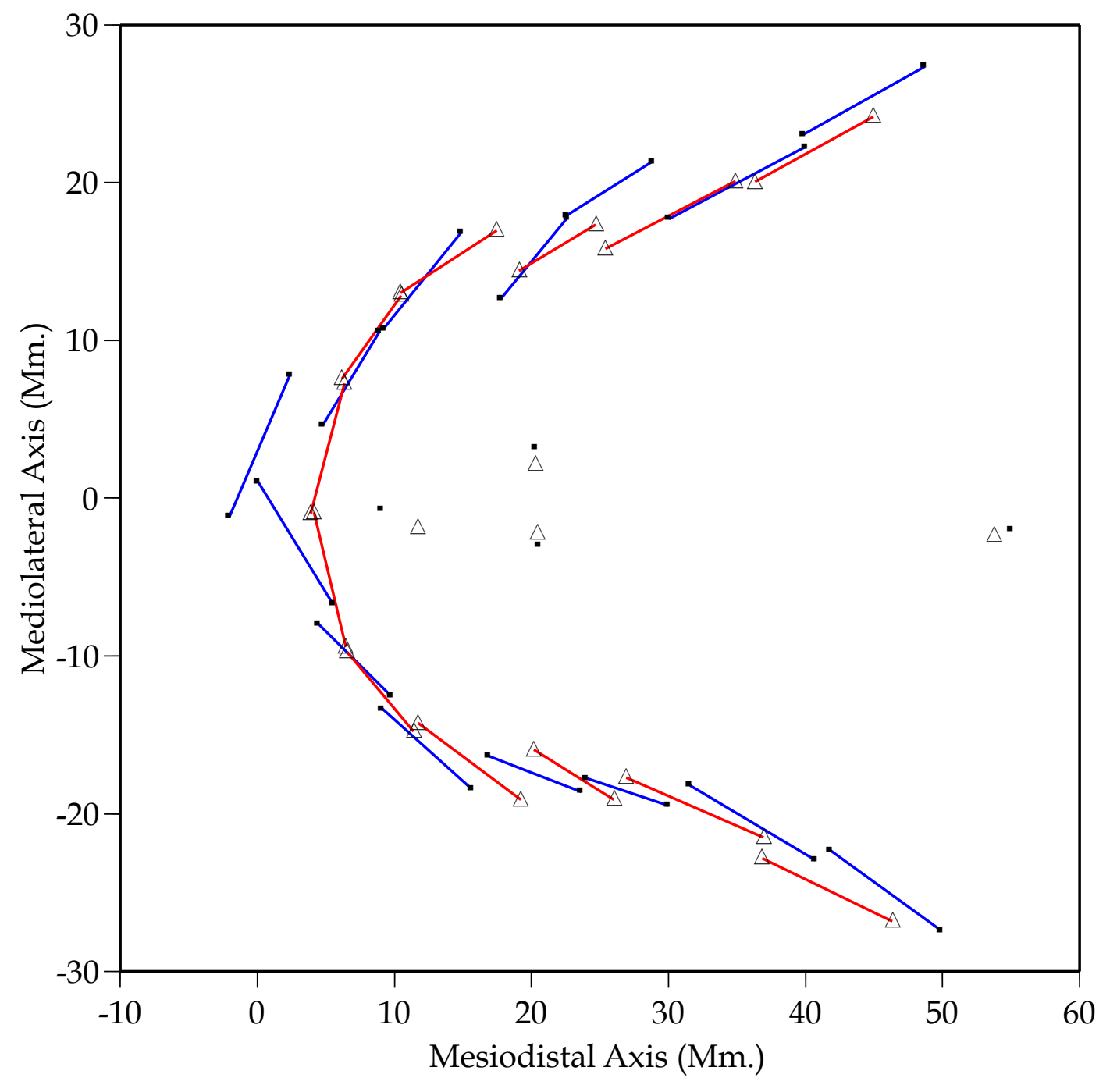

Fig. A-24. Schematic plot of the in-treatment changes in tooth position for case 1664. Registration is on the medial tips of the posterior palatal rugae. Symbols: dots are pretreatment; triangles are posttreatment. 


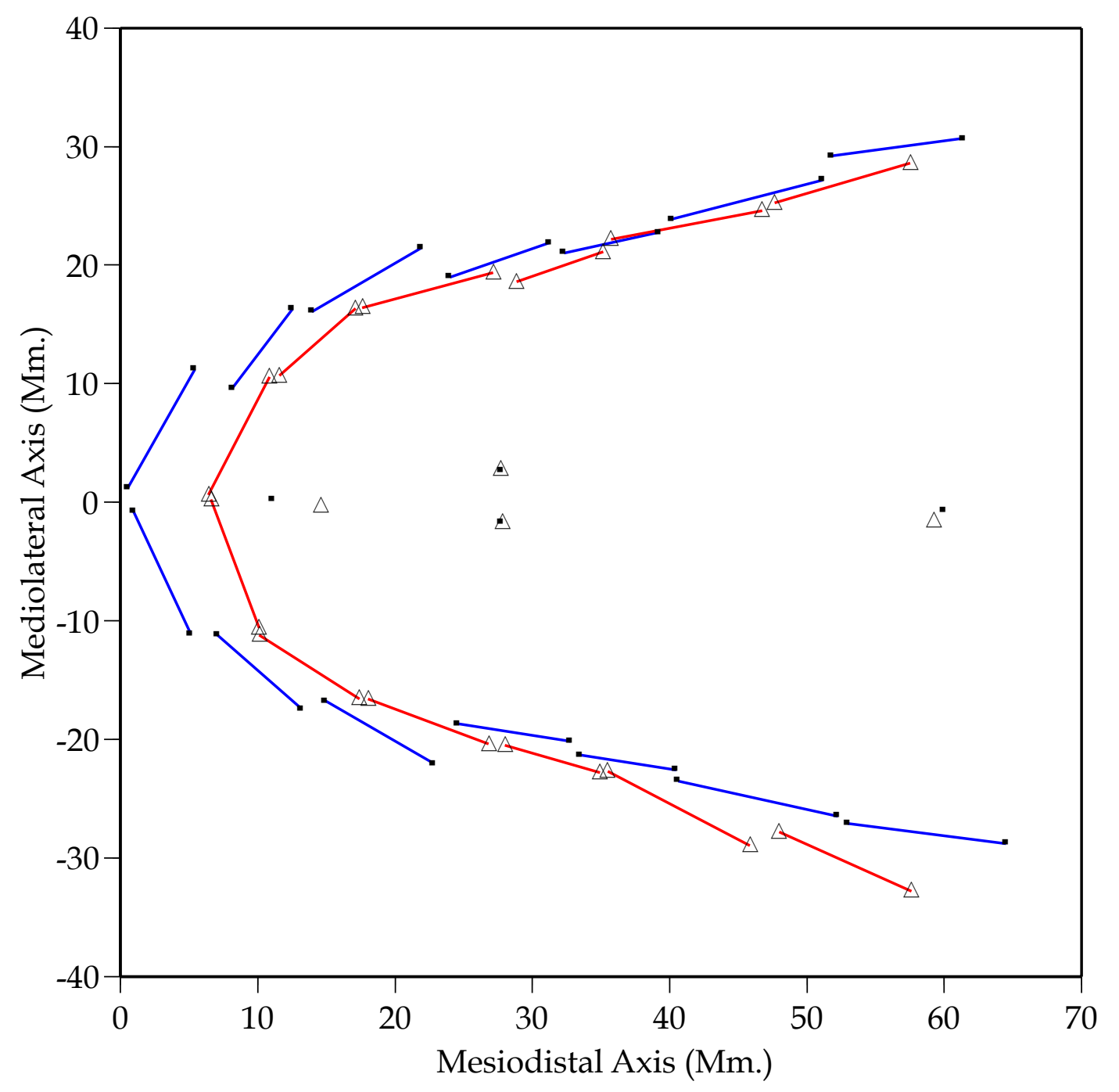

Fig. A-25. Schematic plot of the in-treatment changes in tooth position for case 36. Registration is on the medial tips of the posterior palatal rugae. Symbols: dots are pretreatment; triangles are posttreatment. 


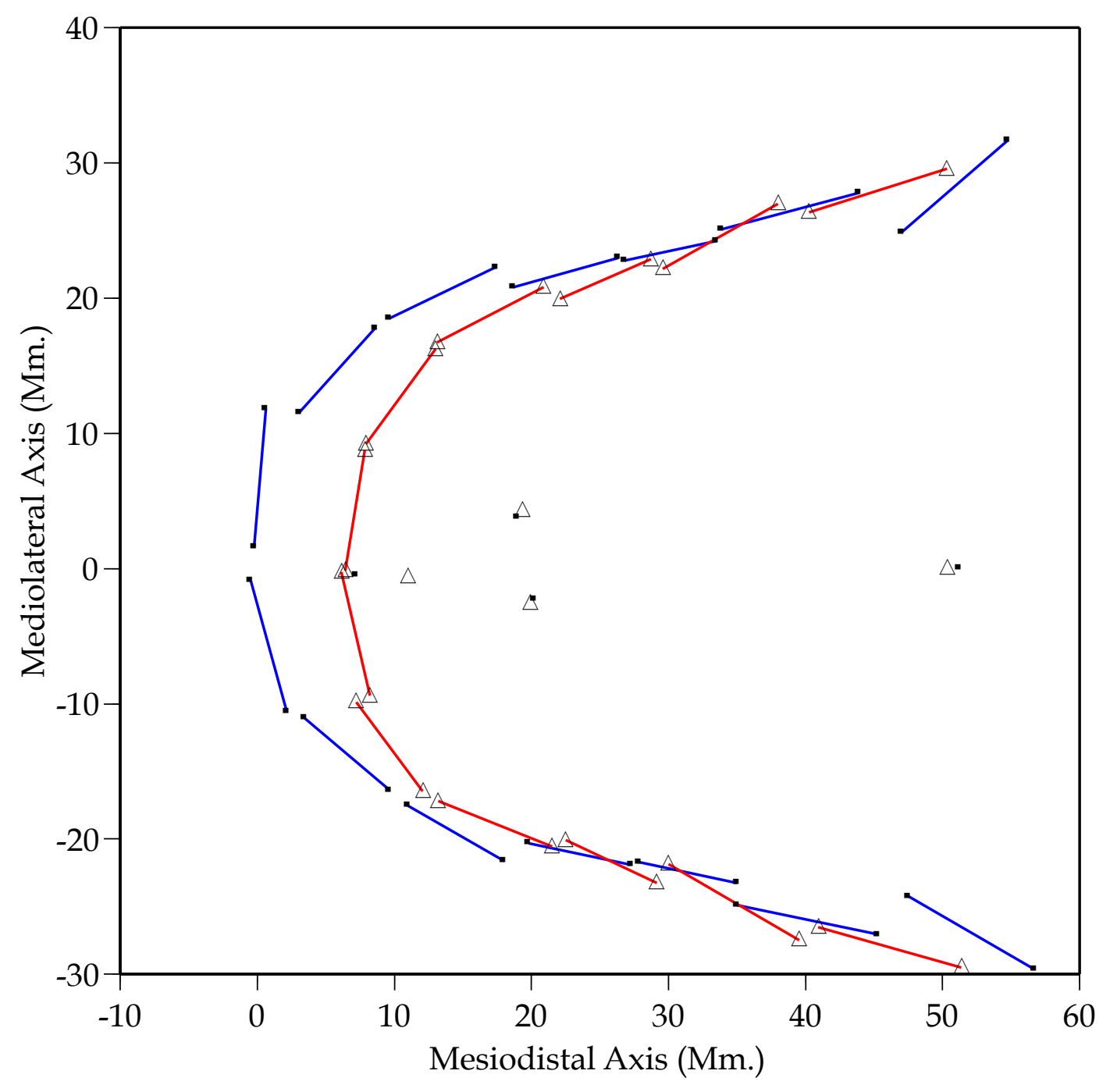

Fig. A-26. Schematic plot of the in-treatment changes in tooth position for case 11. Registration is on the medial tips of the posterior palatal rugae. Symbols: dots are pretreatment; triangles are posttreatment. 


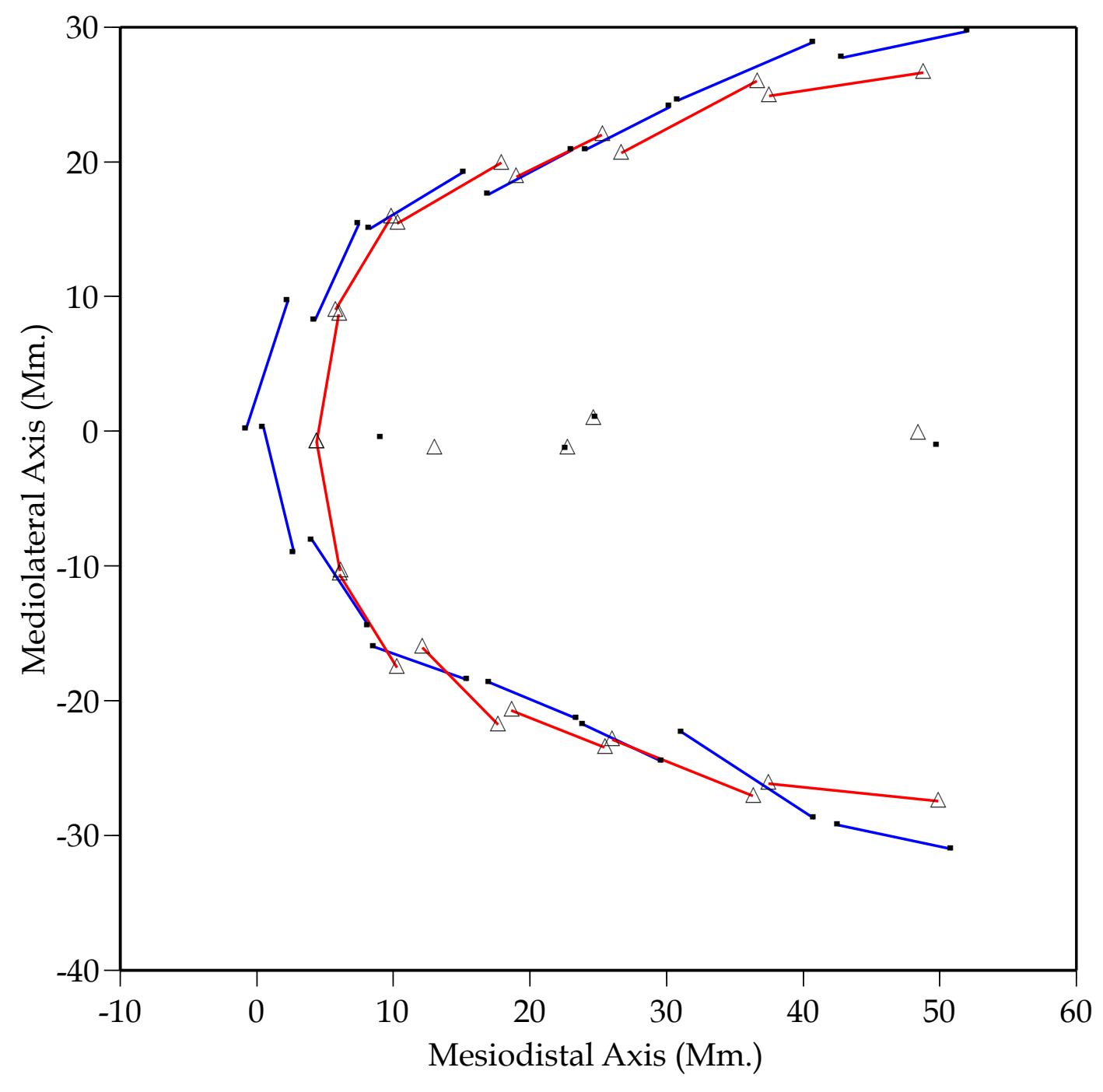

Fig. A-27. Schematic plot of the in-treatment changes in tooth position for case 190. Registration is on the medial tips of the posterior palatal rugae. Symbols: dots are pretreatment; triangles are posttreatment. 


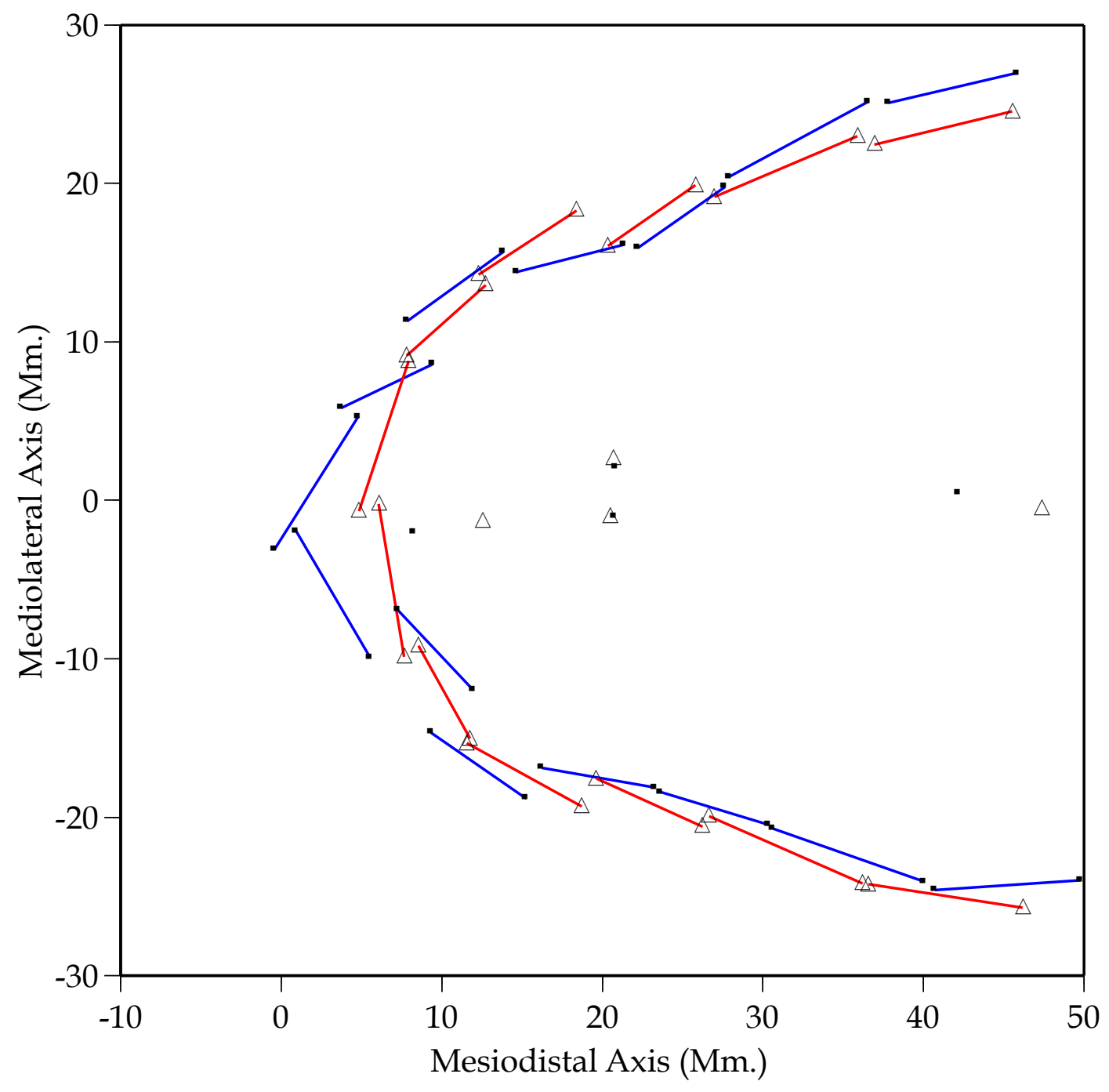

Fig. A-28. Schematic plot of the in-treatment changes in tooth position for case 774. Registration is on the medial tips of the posterior palatal rugae. Symbols: dots are pretreatment; triangles are posttreatment. 


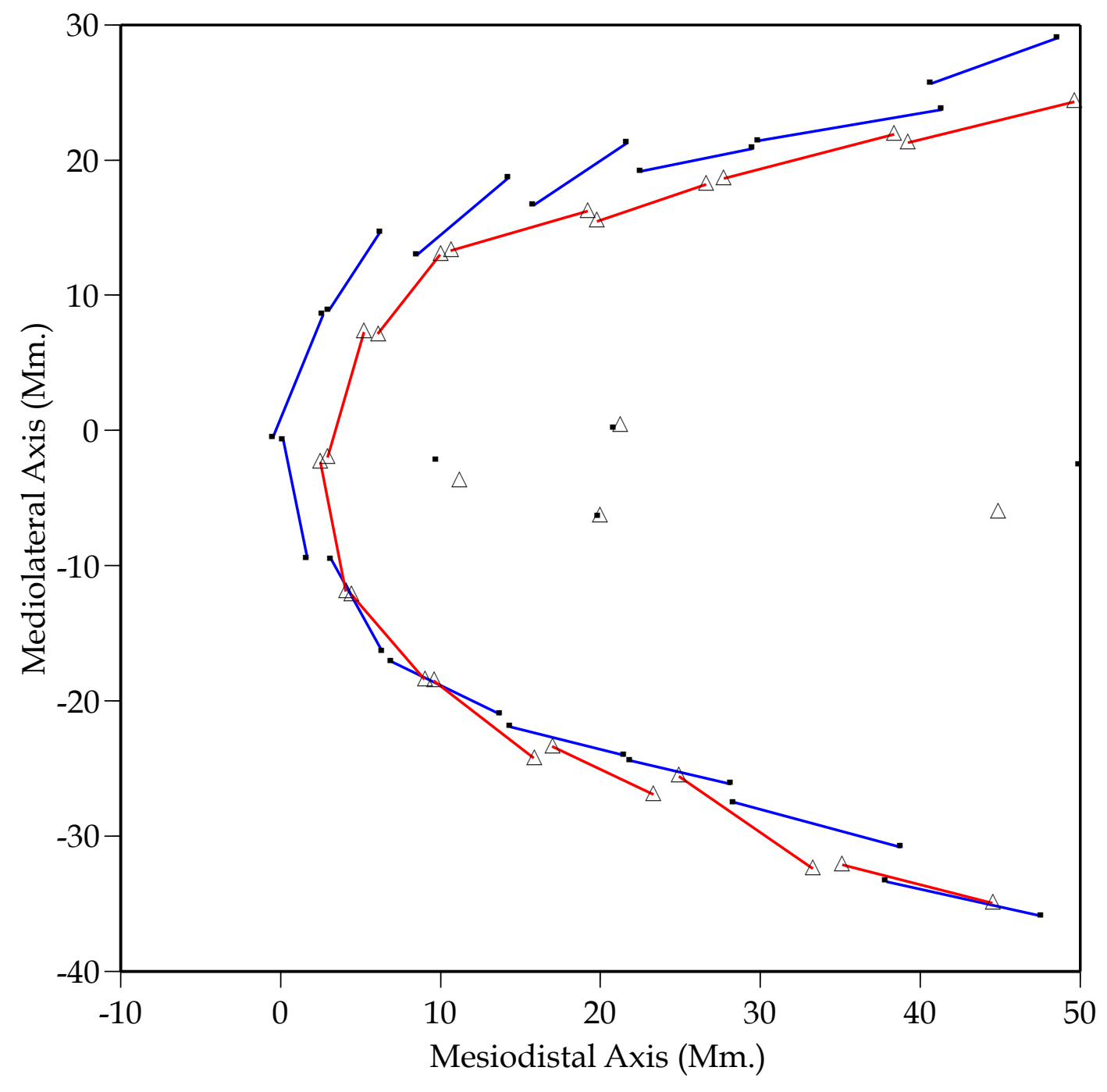

Fig. A-29. Schematic plot of the in-treatment changes in tooth position for case 701. Registration is on the medial tips of the posterior palatal rugae. Symbols: dots are pretreatment; triangles are posttreatment. 


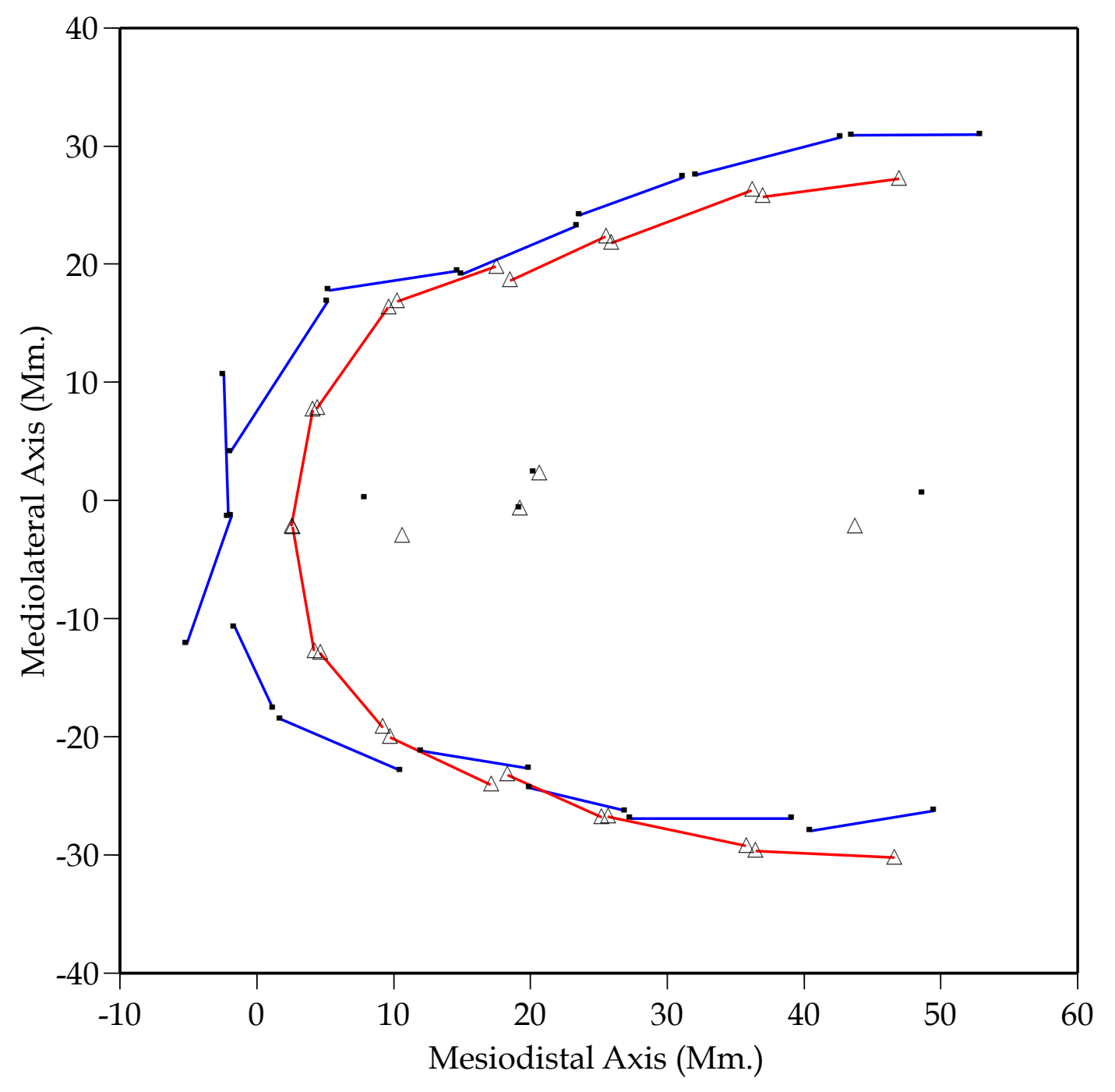

Fig. A-30. Schematic plot of the in-treatment changes in tooth position for case 1970. Registration is on the medial tips of the posterior palatal rugae. Symbols: dots are pretreatment; triangles are posttreatment. 


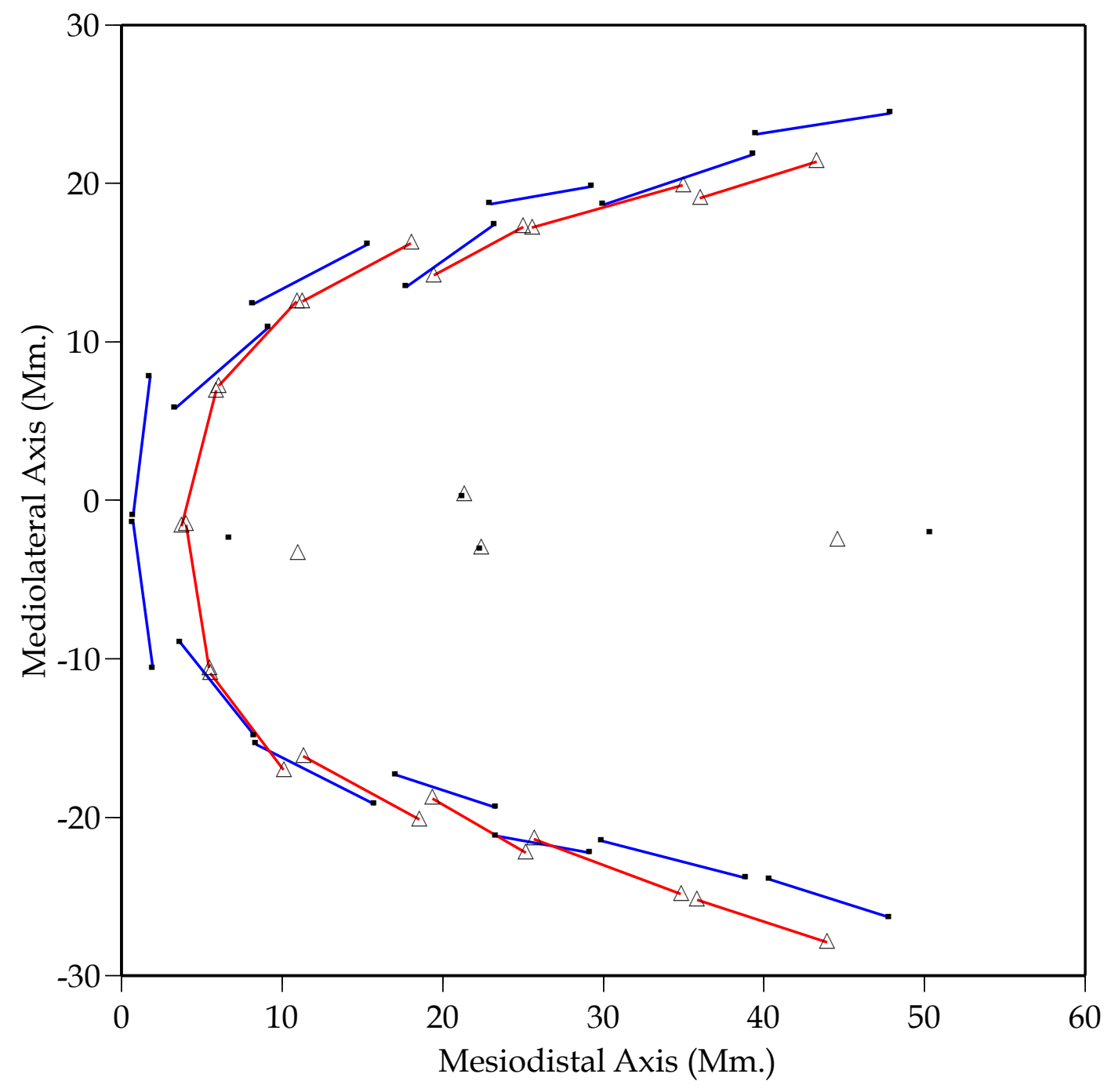

Fig. A-31. Schematic plot of the in-treatment changes in tooth position for case 1291. Registration is on the medial tips of the posterior palatal rugae. Symbols: dots are pretreatment; triangles are posttreatment. 


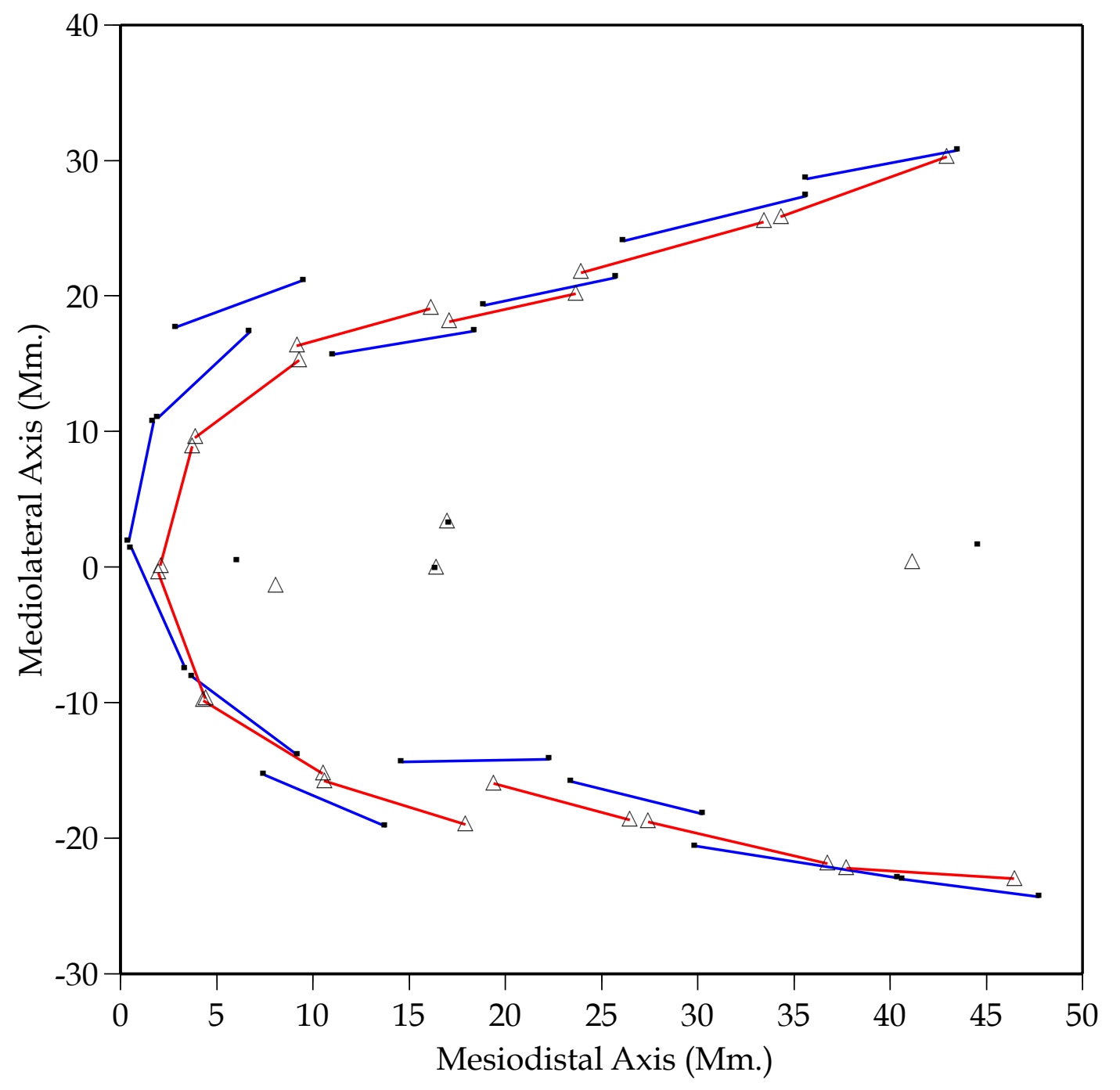

Fig. A-32. Schematic plot of the in-treatment changes in tooth position for case 1213. Registration is on the medial tips of the posterior palatal rugae. Symbols: dots are pretreatment; triangles are posttreatment. 


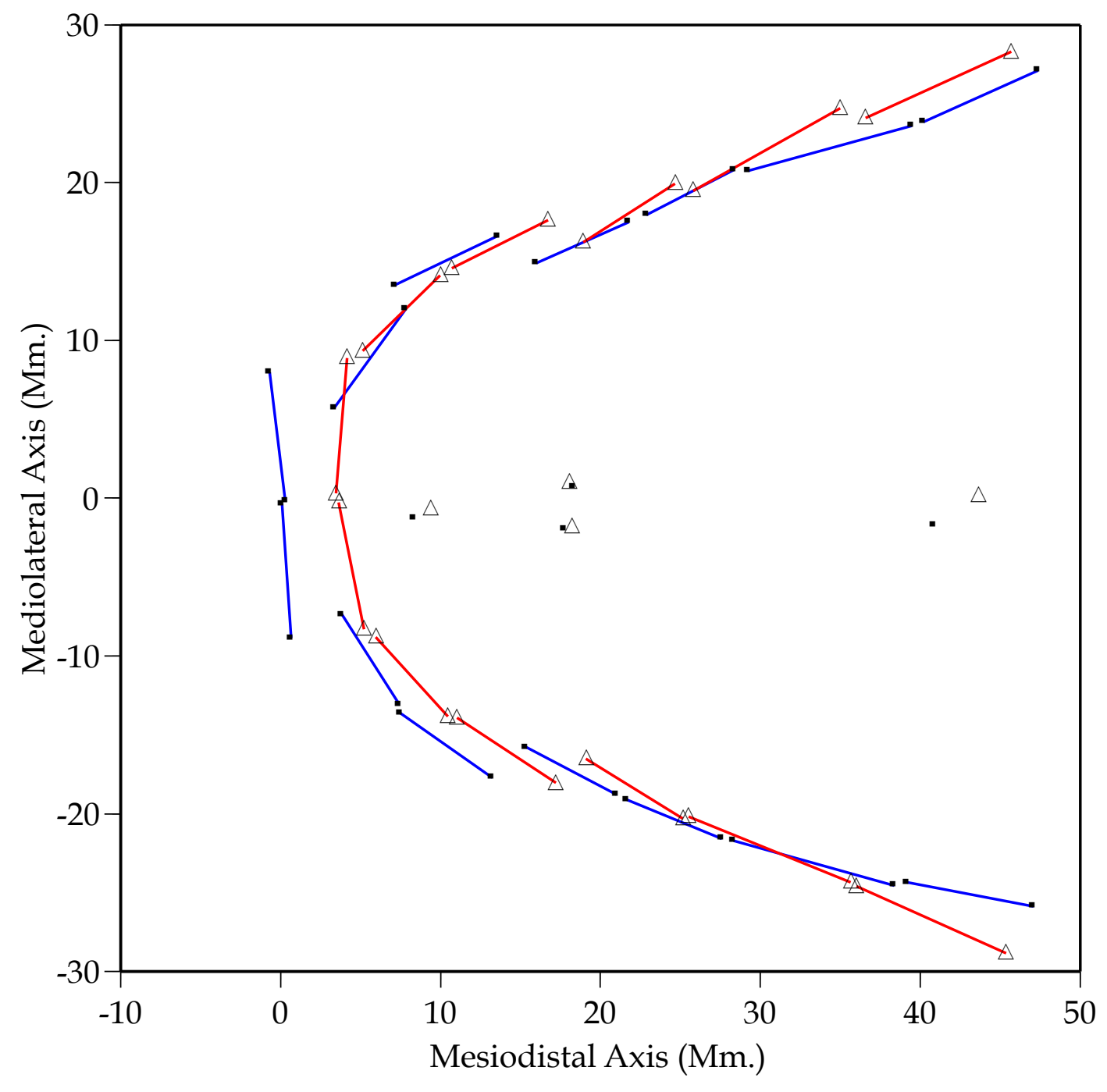

Fig. A-33. Schematic plot of the in-treatment changes in tooth position for case 366. Registration is on the medial tips of the posterior palatal rugae. Symbols: dots are pretreatment; triangles are posttreatment. 


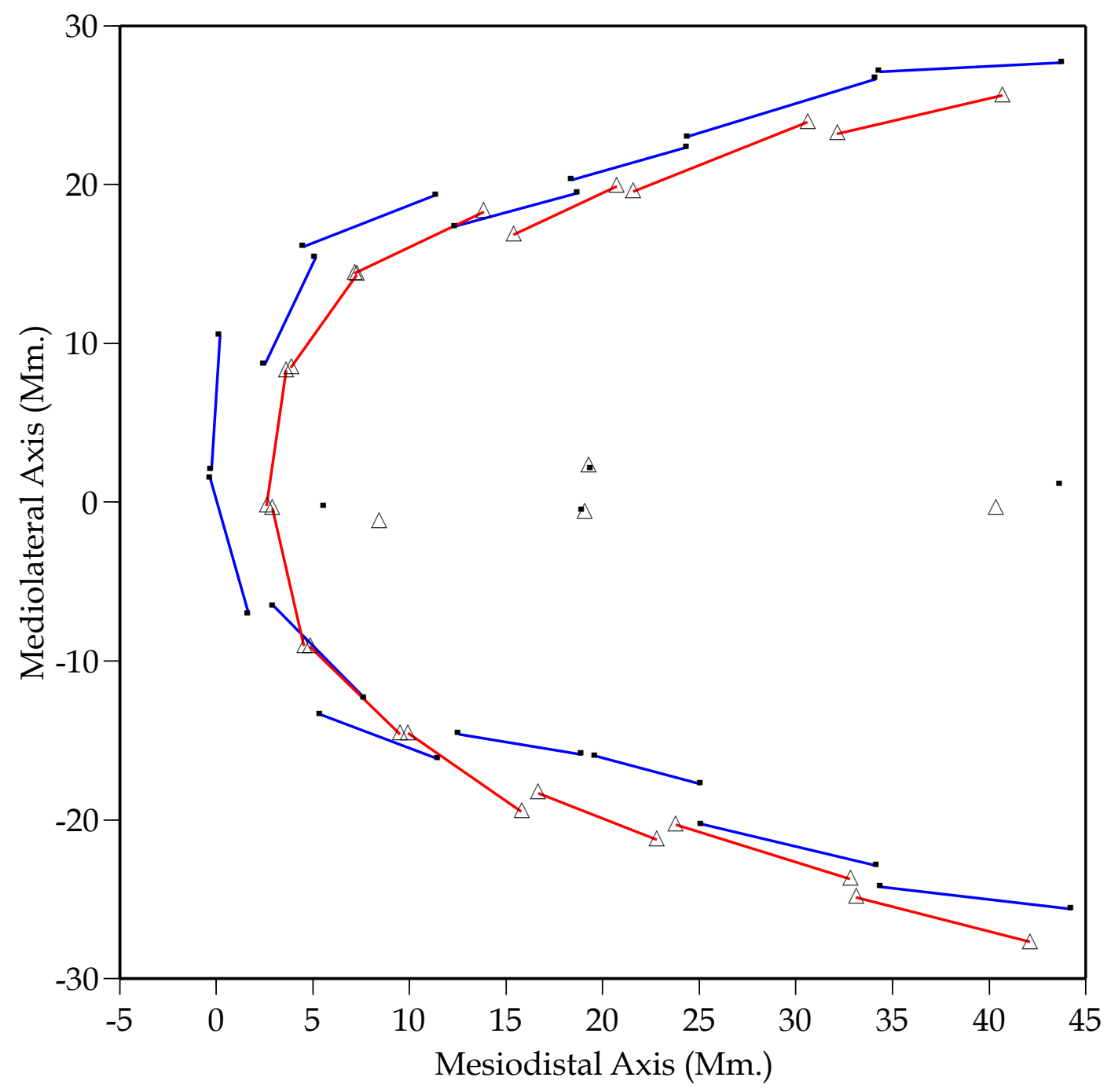

Fig. A-34. Schematic plot of the in-treatment changes in tooth position for case 176. Registration is on the medial tips of the posterior palatal rugae. Symbols: dots are pretreatment; triangles are posttreatment. 


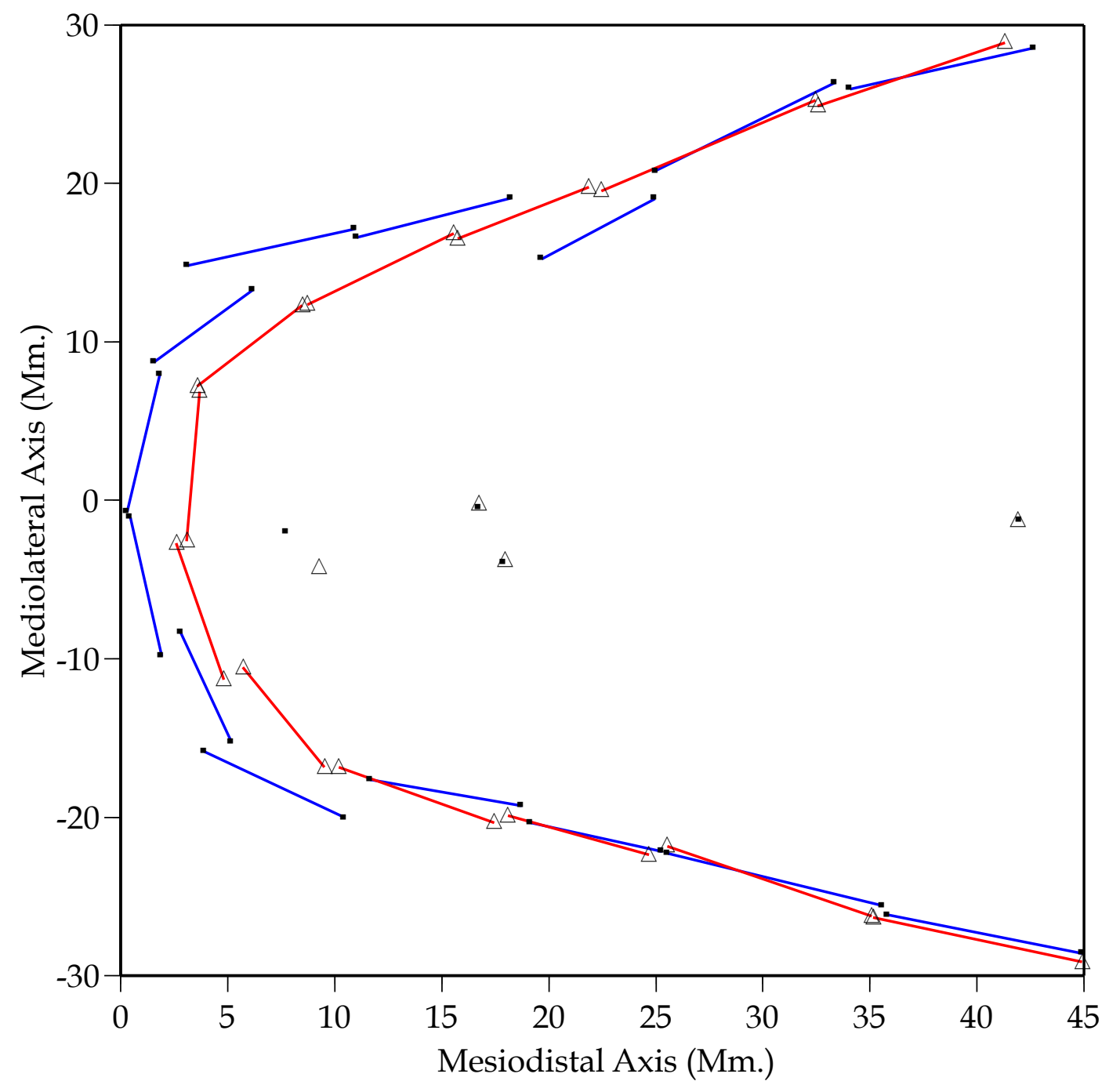

Fig. A-35. Schematic plot of the in-treatment changes in tooth position for case 772. Registration is on the medial tips of the posterior palatal rugae. Symbols: dots are pretreatment; triangles are posttreatment. 


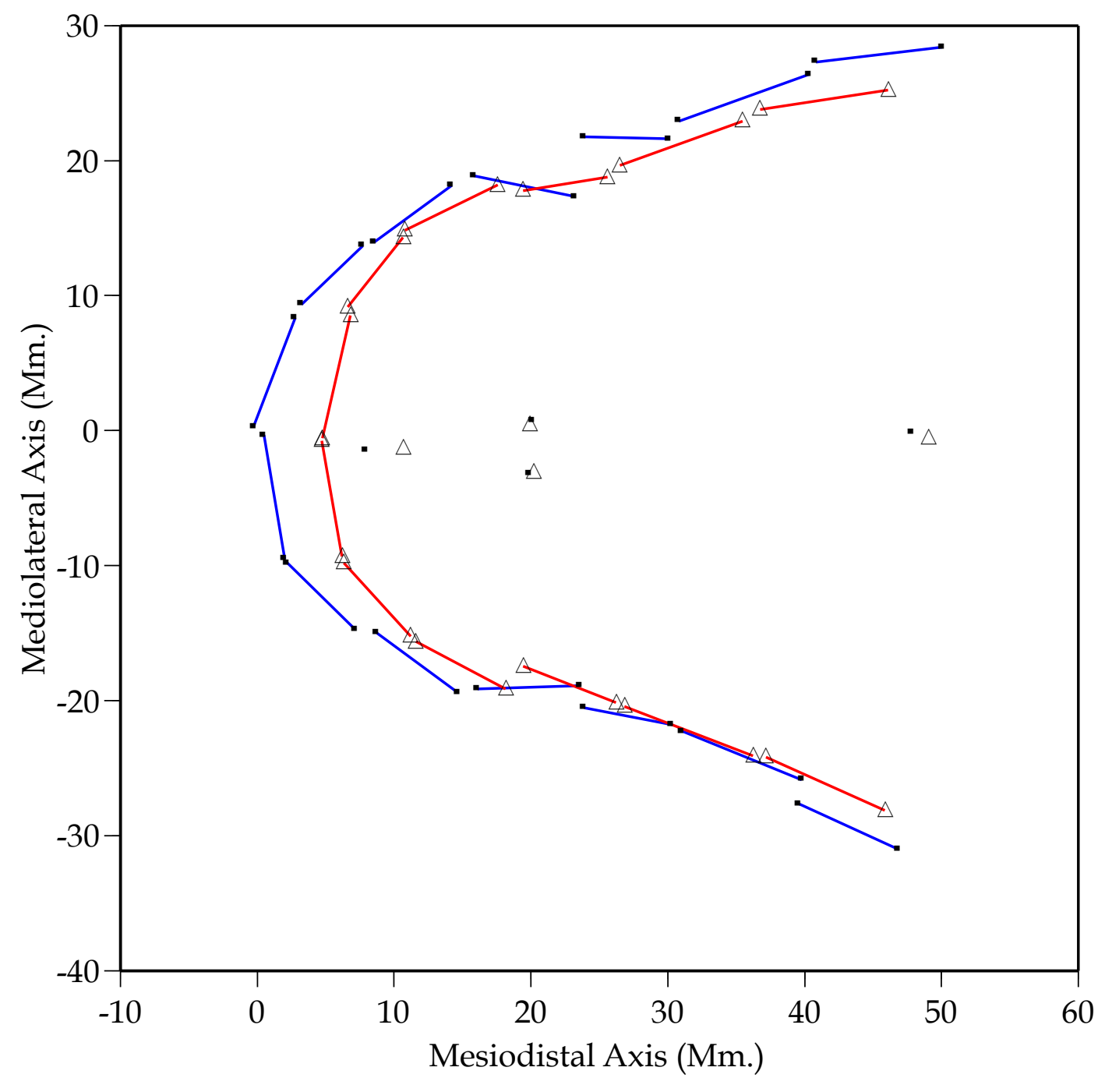

Fig. A-36 Schematic plot of the in-treatment changes in tooth position for case 1575. Registration is on the medial tips of the posterior palatal rugae. Symbols: dots are pretreatment; triangles are posttreatment. 


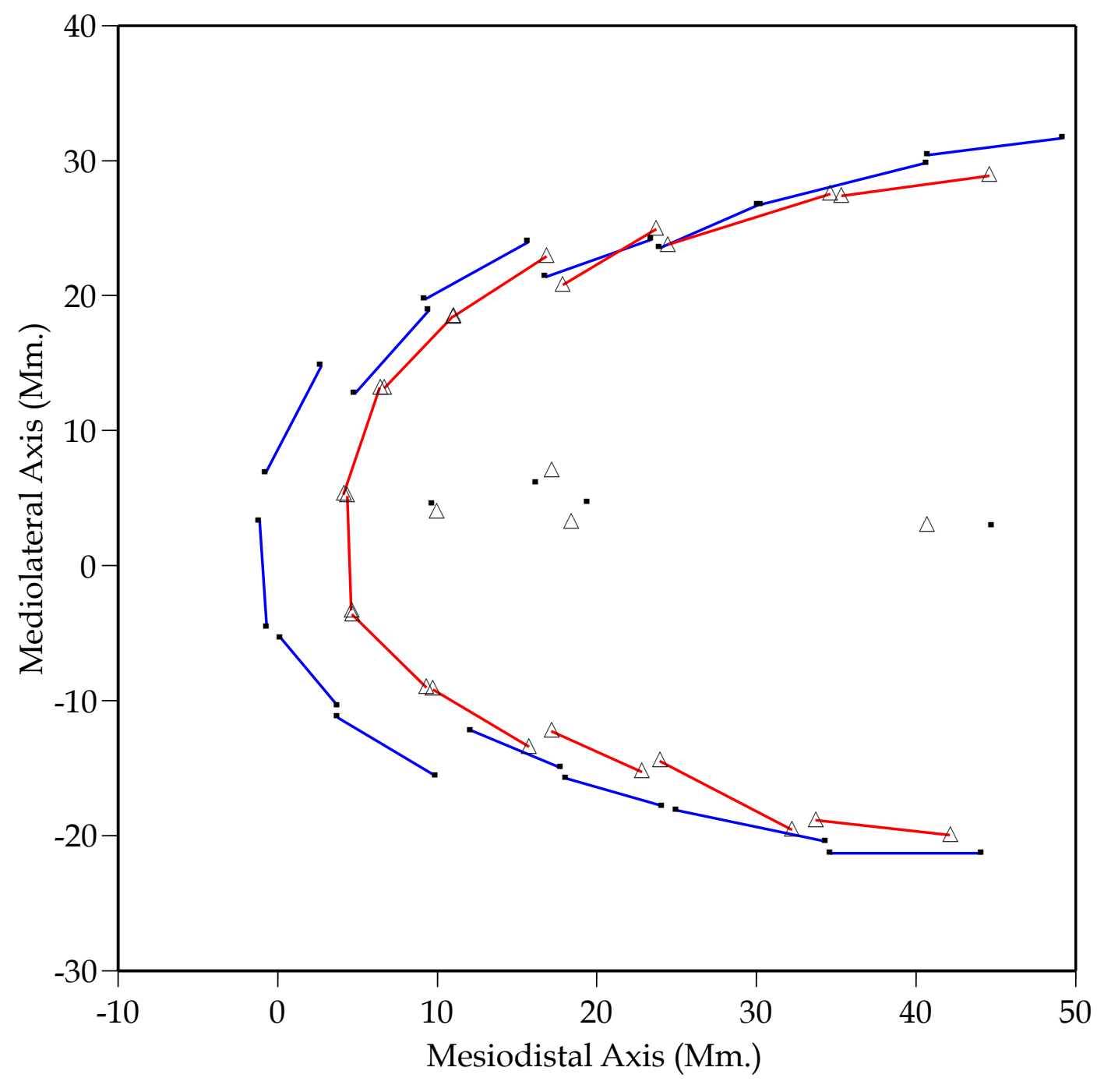

Fig. A-37. Schematic plot of the in-treatment changes in tooth position for case 1340. Registration is on the medial tips of the posterior palatal rugae. Symbols: dots are pretreatment; triangles are posttreatment. 


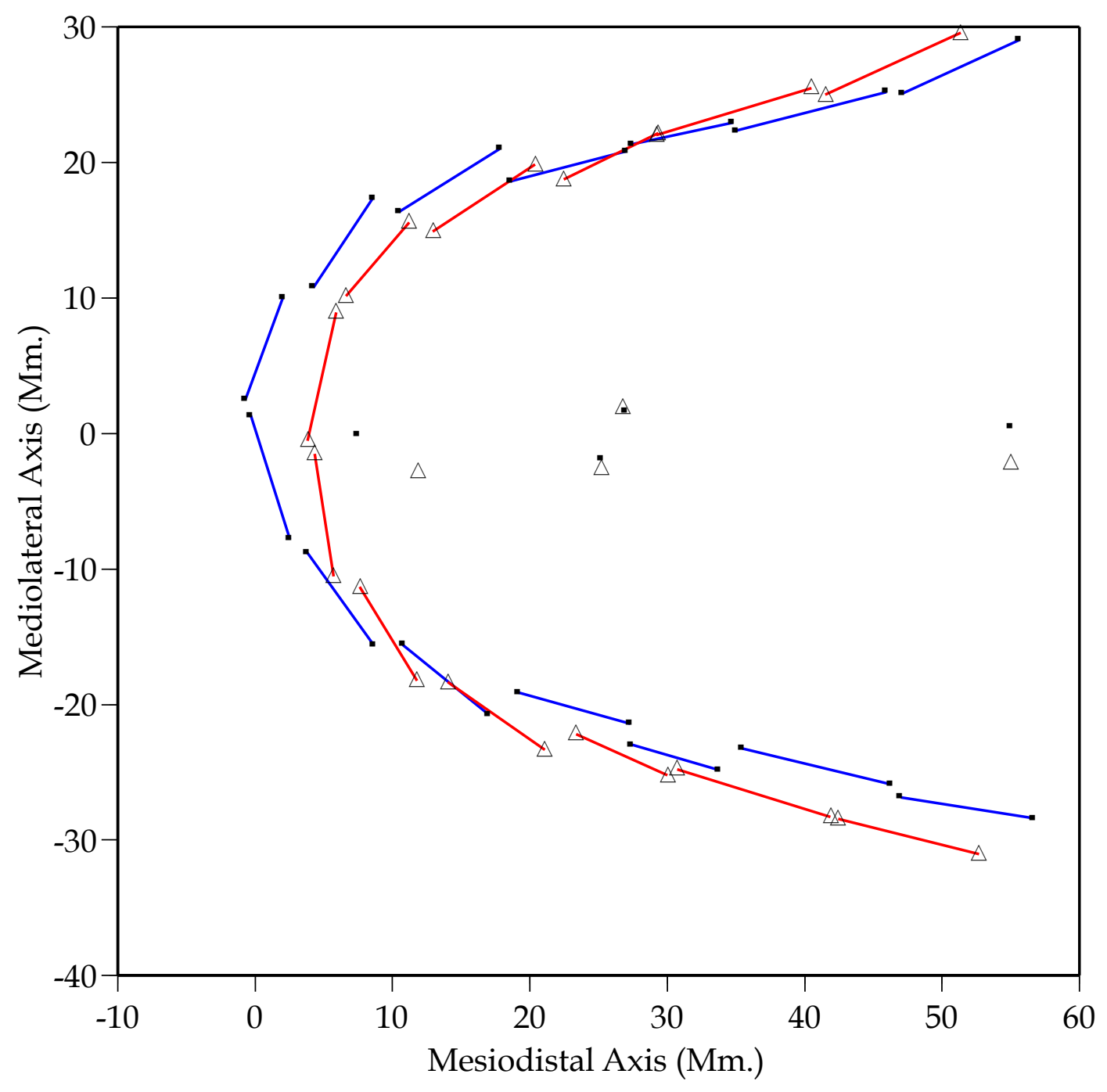

Fig. A-38. Schematic plot of the in-treatment changes in tooth position for case 1640. Registration is on the medial tips of the posterior palatal rugae. Symbols: dots are pretreatment; triangles are posttreatment. 


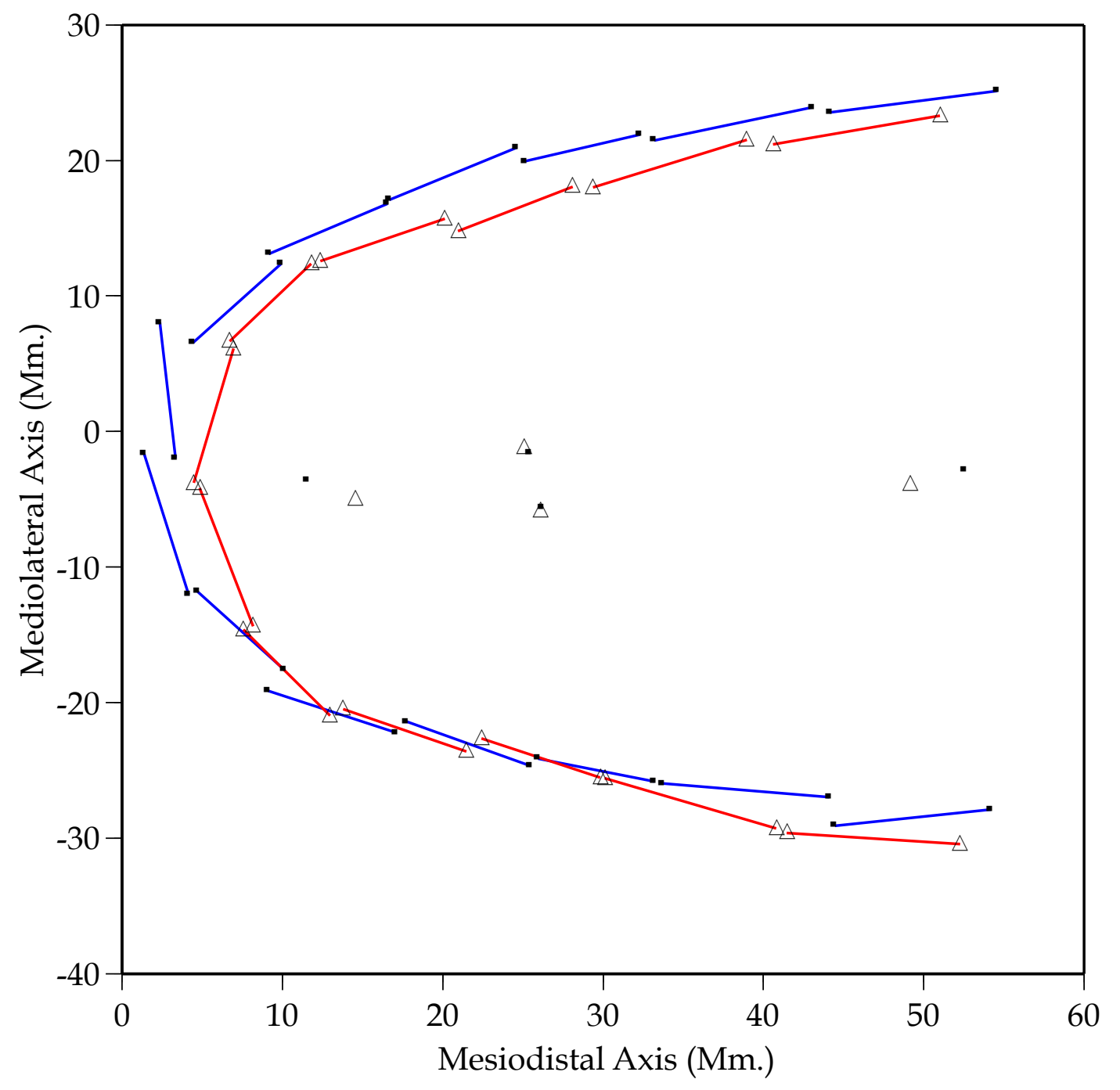

Fig. A-39. Schematic plot of the in-treatment changes in tooth position for case 1262. Registration is on the medial tips of the posterior palatal rugae. Symbols: dots are pretreatment; triangles are posttreatment. 


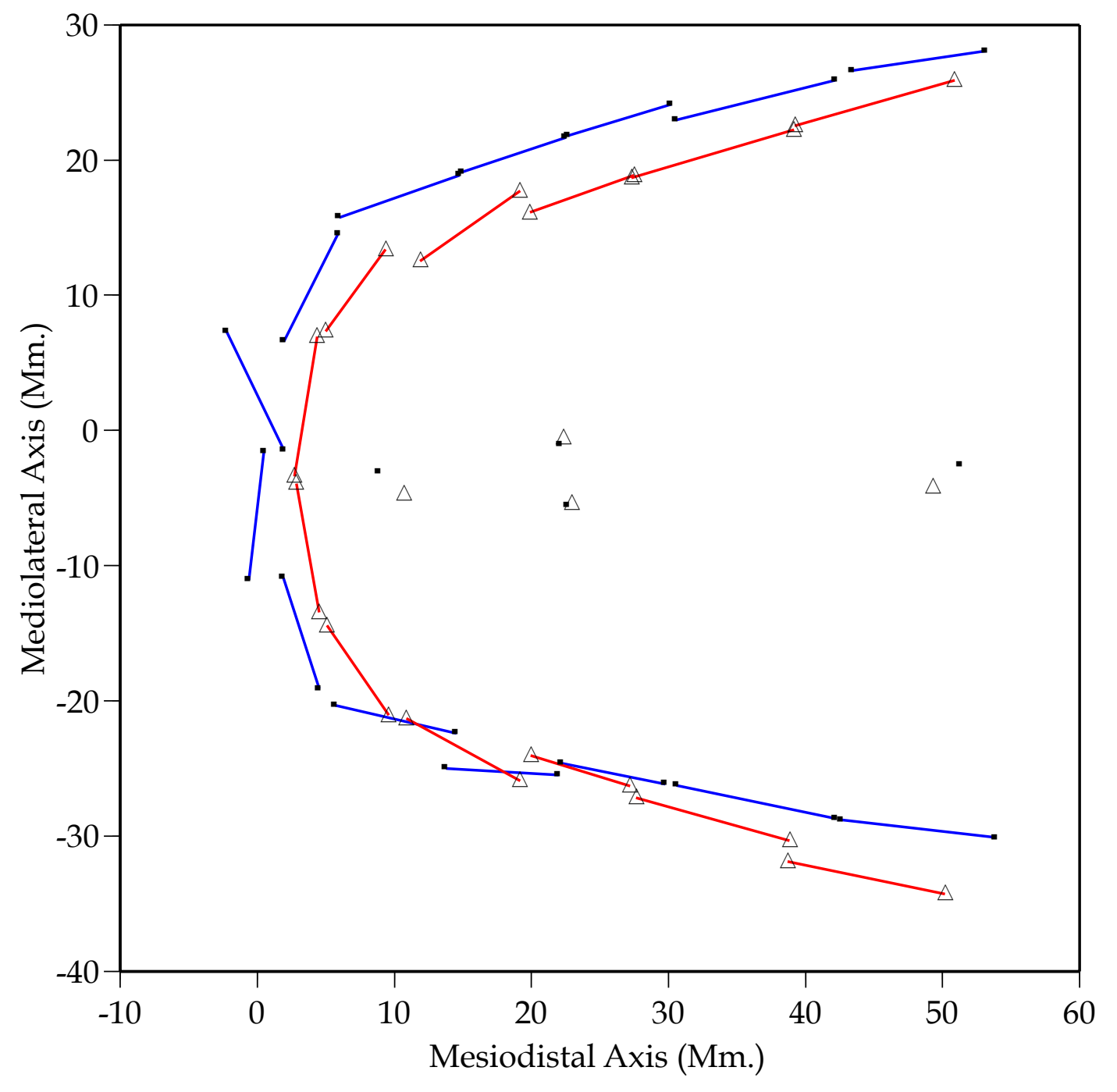

Fig. A-40. Schematic plot of the in-treatment changes in tooth position for case 1971. Registration is on the medial tips of the posterior palatal rugae. Symbols: dots are pretreatment; triangles are posttreatment. 


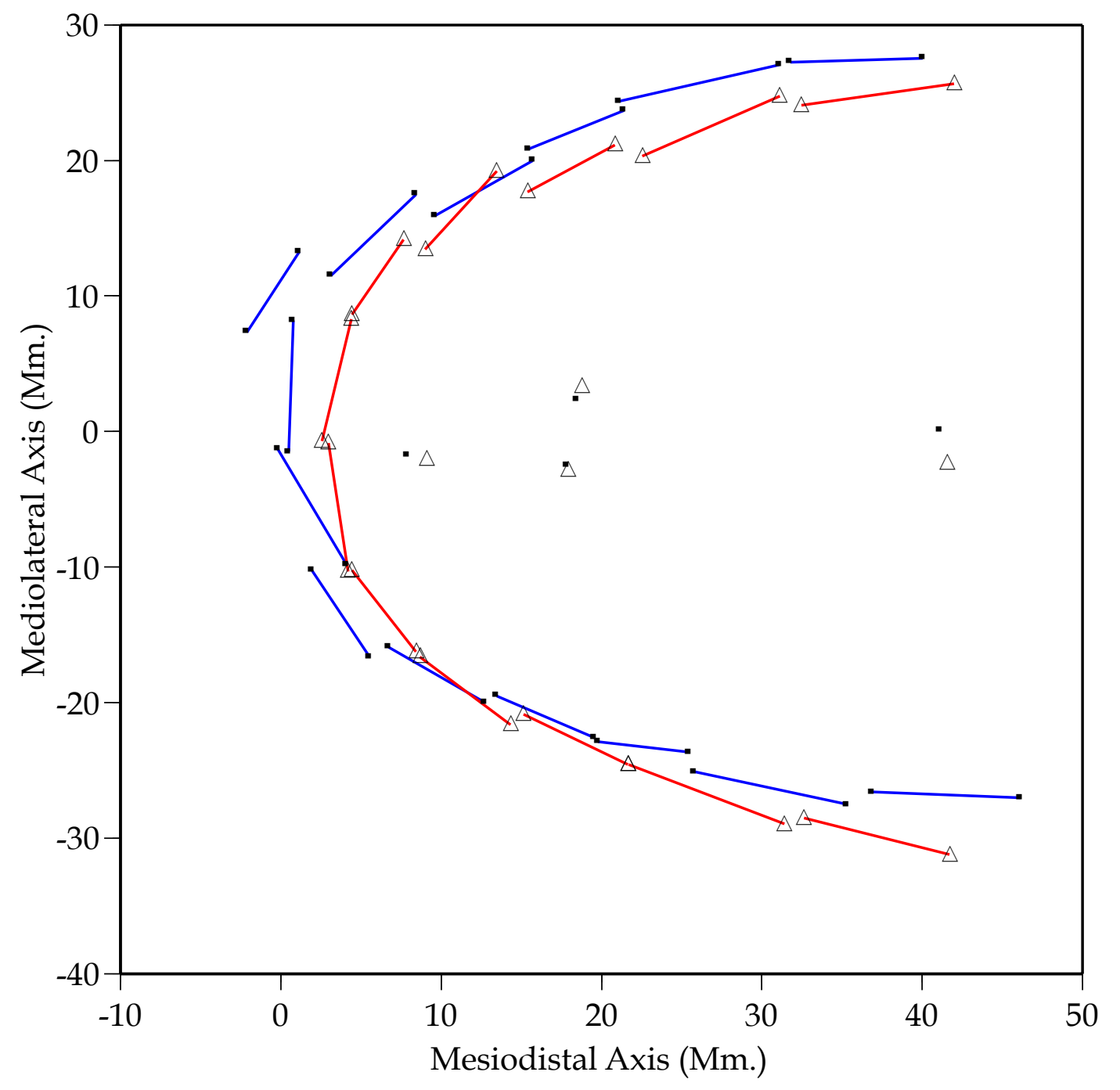

Fig. A-41. Schematic plot of the in-treatment changes in tooth position for case 1257. Registration is on the medial tips of the posterior palatal rugae. Symbols: dots are pretreatment; triangles are posttreatment. 


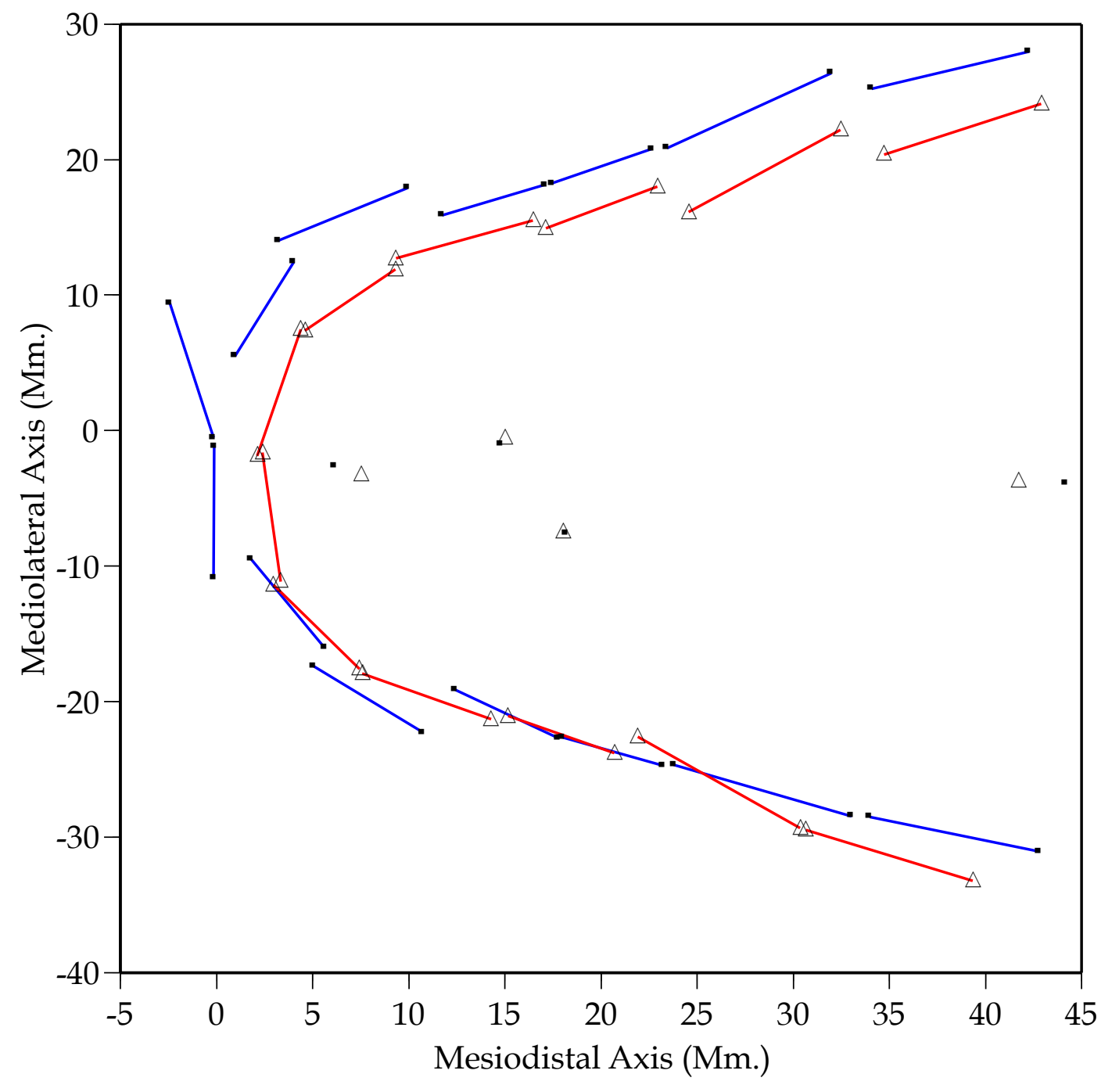

Fig. A-42. Schematic plot of the in-treatment changes in tooth position for case 1382. Registration is on the medial tips of the posterior palatal rugae. Symbols: dots are pretreatment; triangles are posttreatment. 


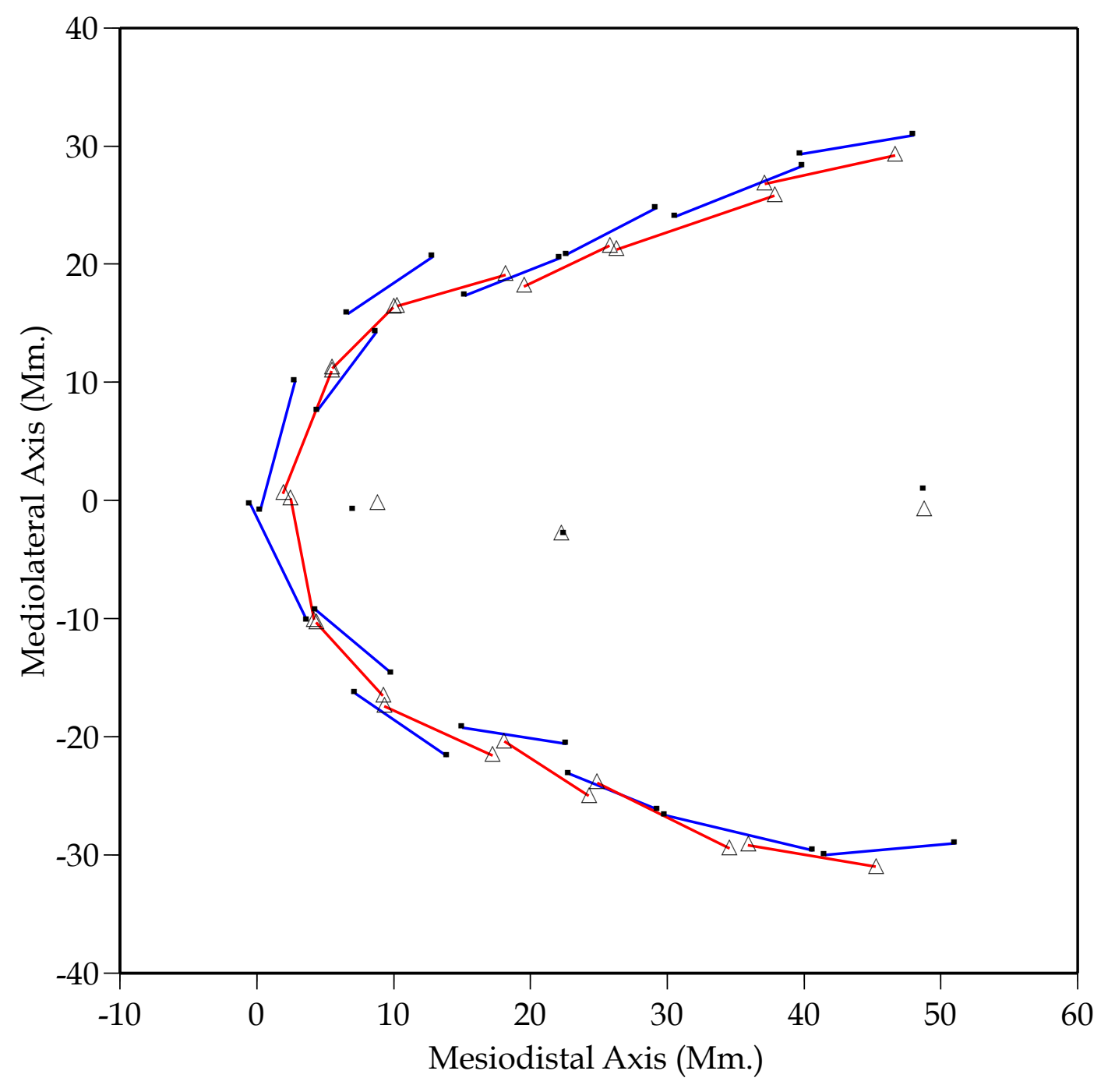

Fig. A-43. Schematic plot of the in-treatment changes in tooth position for case 1661. Registration is on the medial tips of the posterior palatal rugae. Symbols: dots are pretreatment; triangles are posttreatment. 


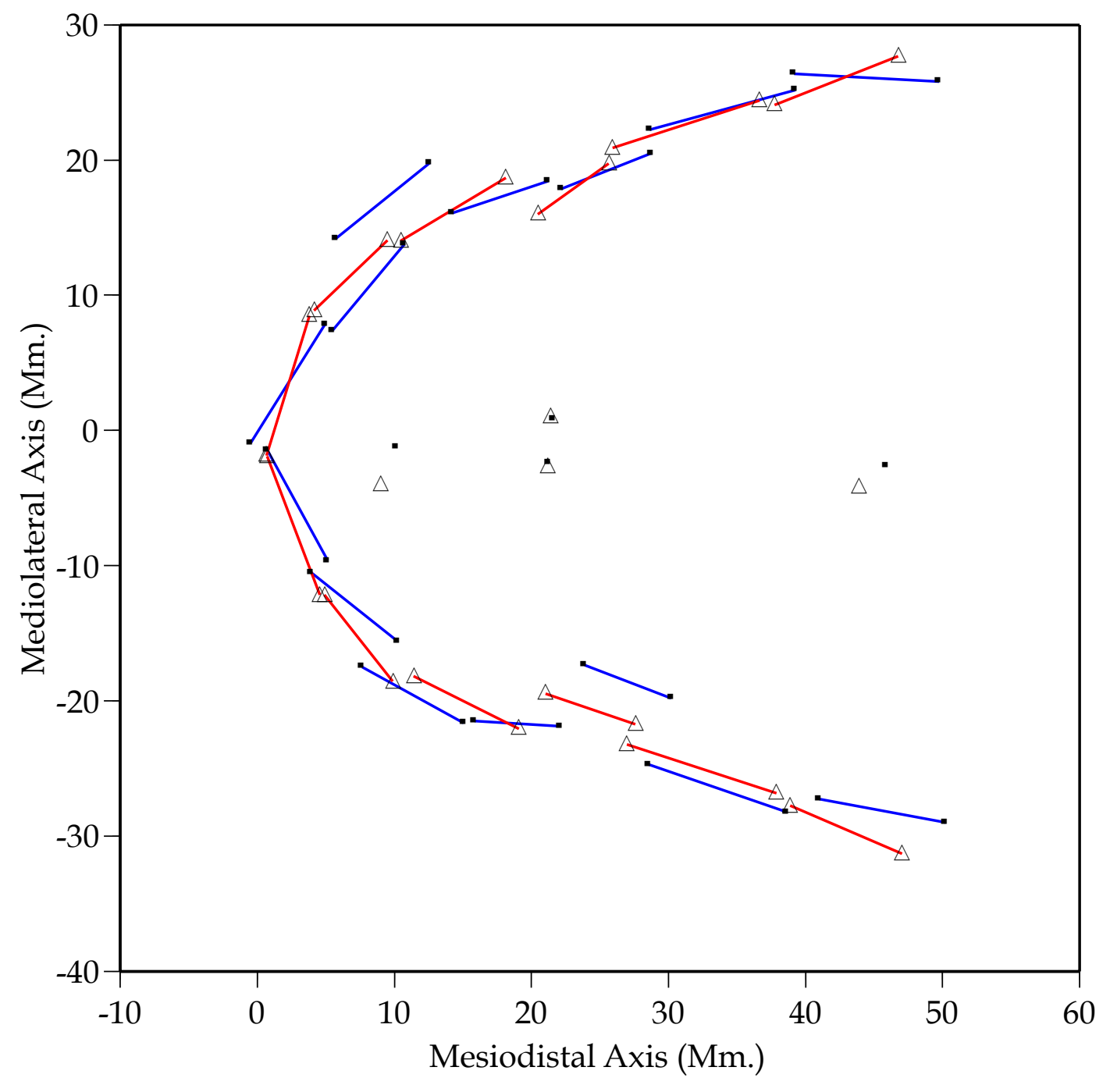

Fig. A-44. Schematic plot of the in-treatment changes in tooth position for case 325. Registration is on the medial tips of the posterior palatal rugae. Symbols: dots are pretreatment; triangles are posttreatment. 


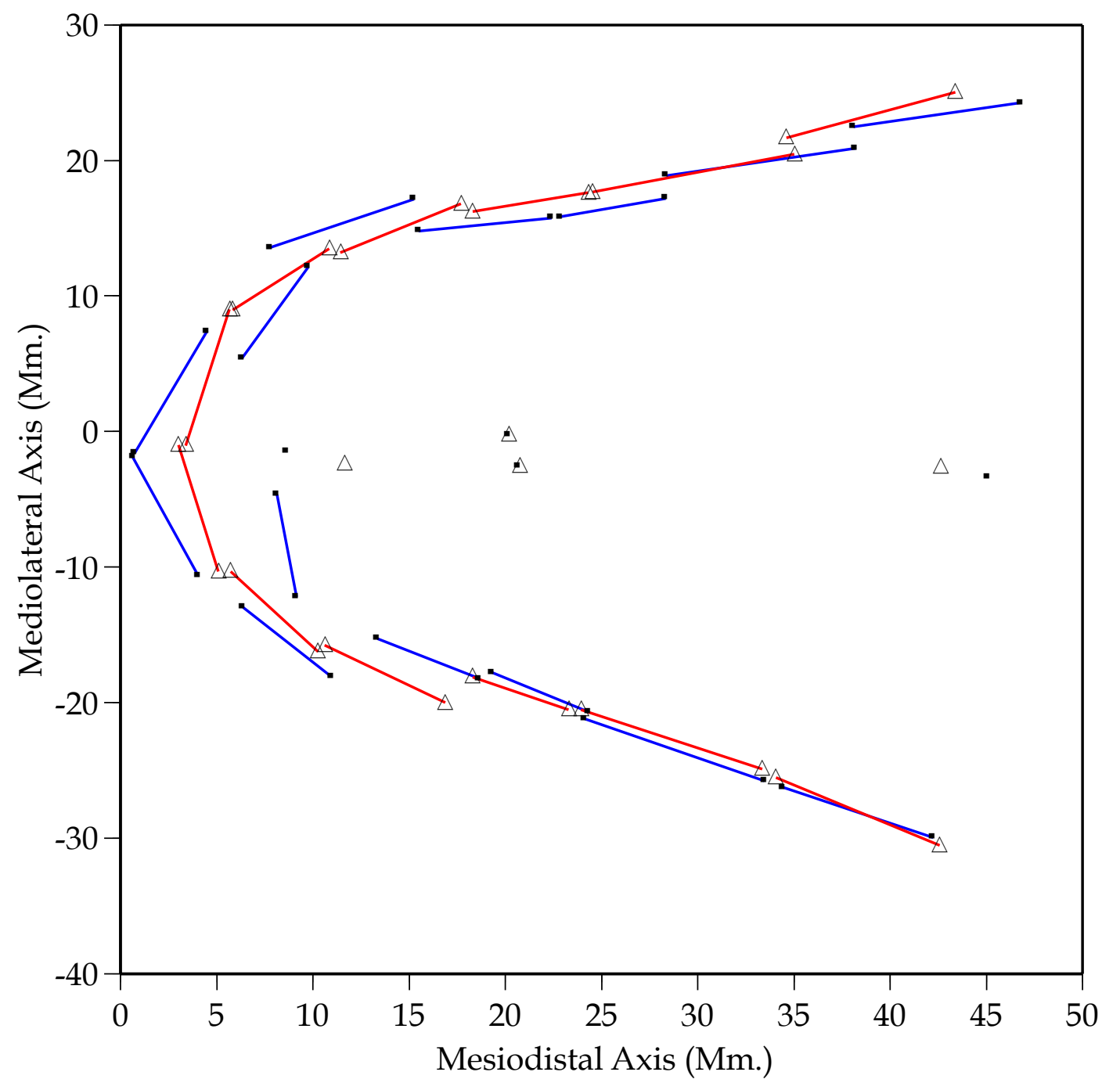

Fig. A-45. Schematic plot of the in-treatment changes in tooth position for case 703. Registration is on the medial tips of the posterior palatal rugae. Symbols: dots are pretreatment; triangles are posttreatment. 


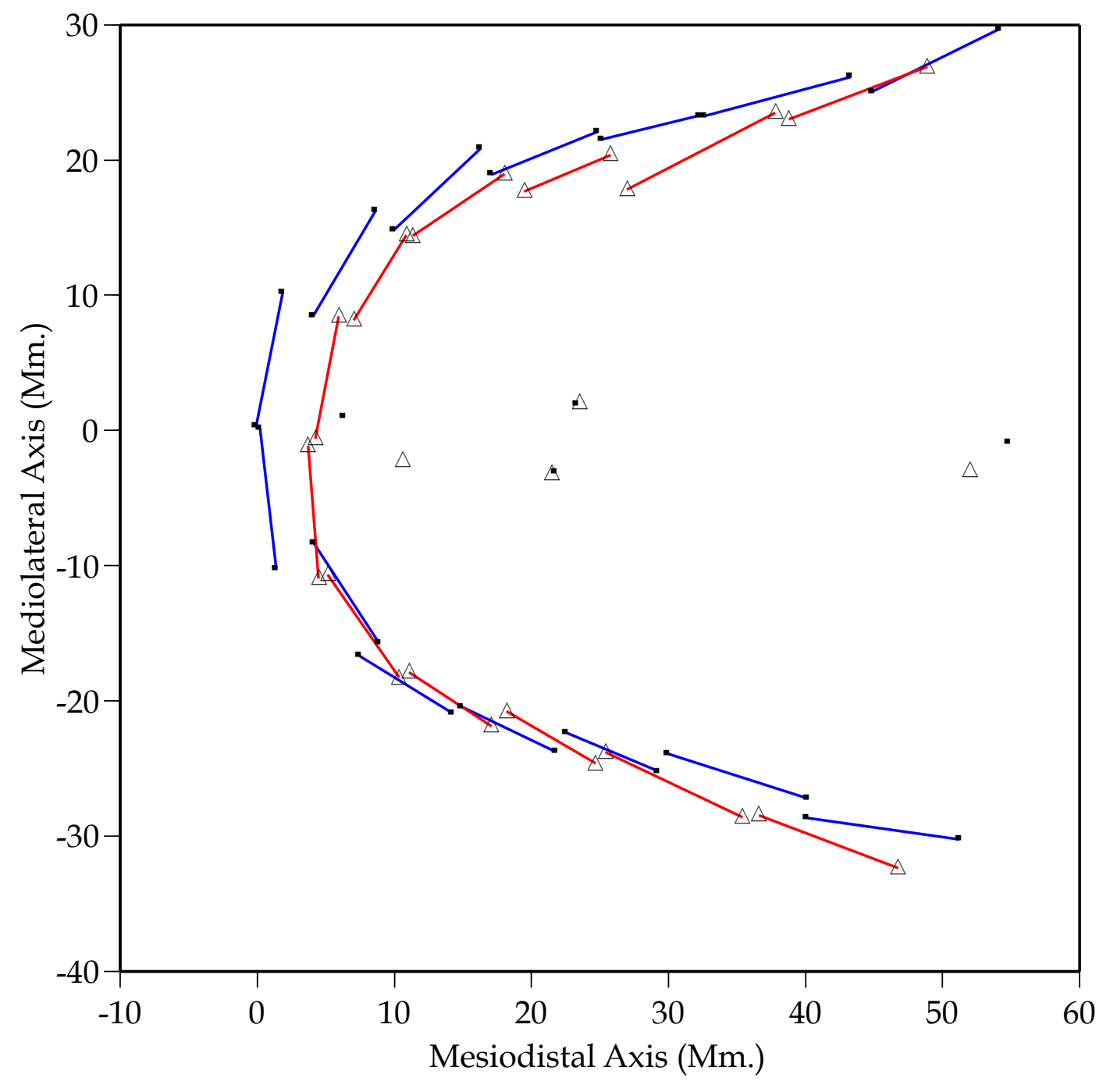

Fig. A-46. Schematic plot of the in-treatment changes in tooth position for case 2390. Registration is on the medial tips of the posterior palatal rugae. Symbols: dots are pretreatment; triangles are posttreatment. 


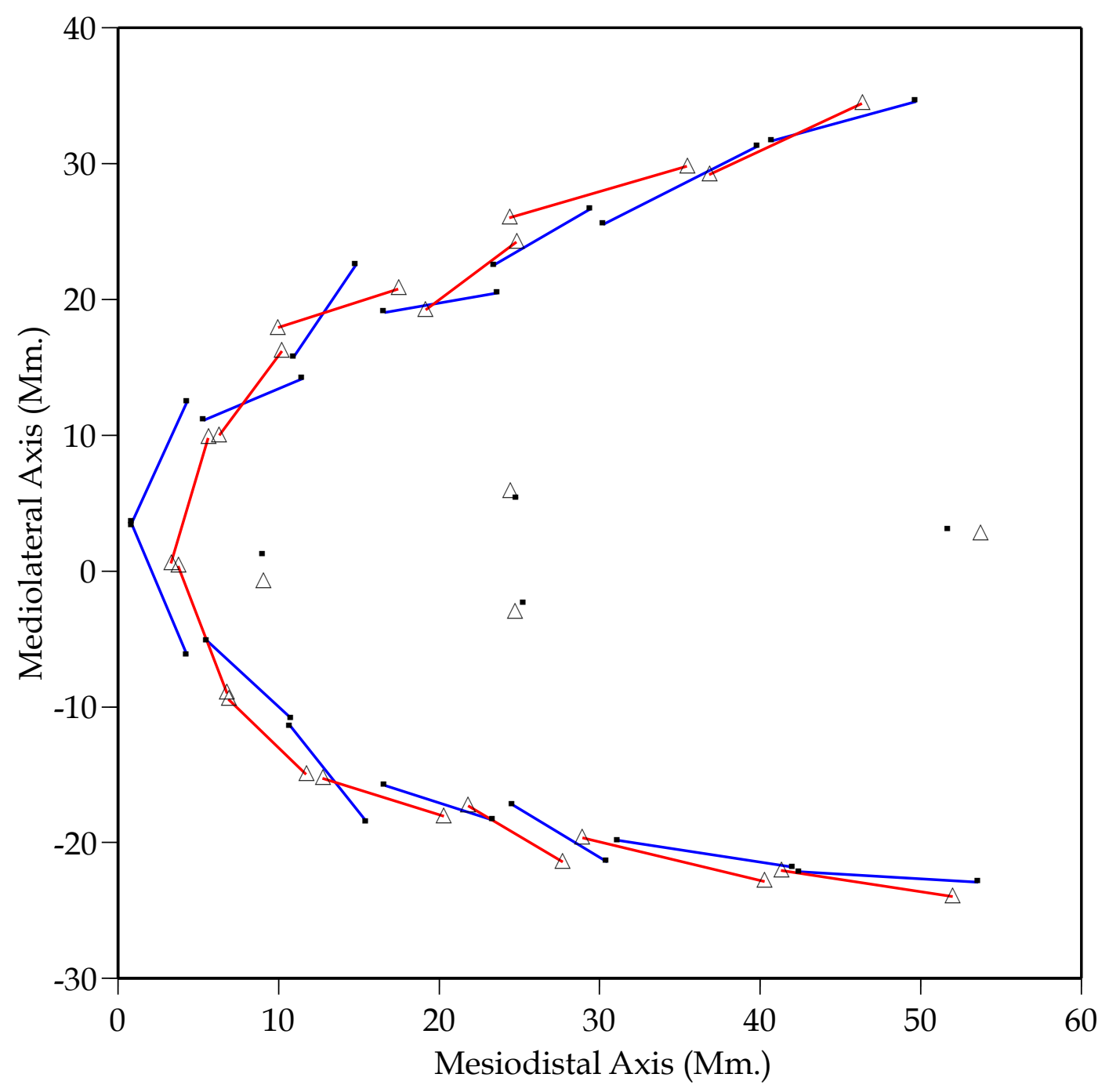

Fig. A-47. Schematic plot of the in-treatment changes in tooth position for case 1668. Registration is on the medial tips of the posterior palatal rugae. Symbols: dots are pretreatment; triangles are posttreatment. 


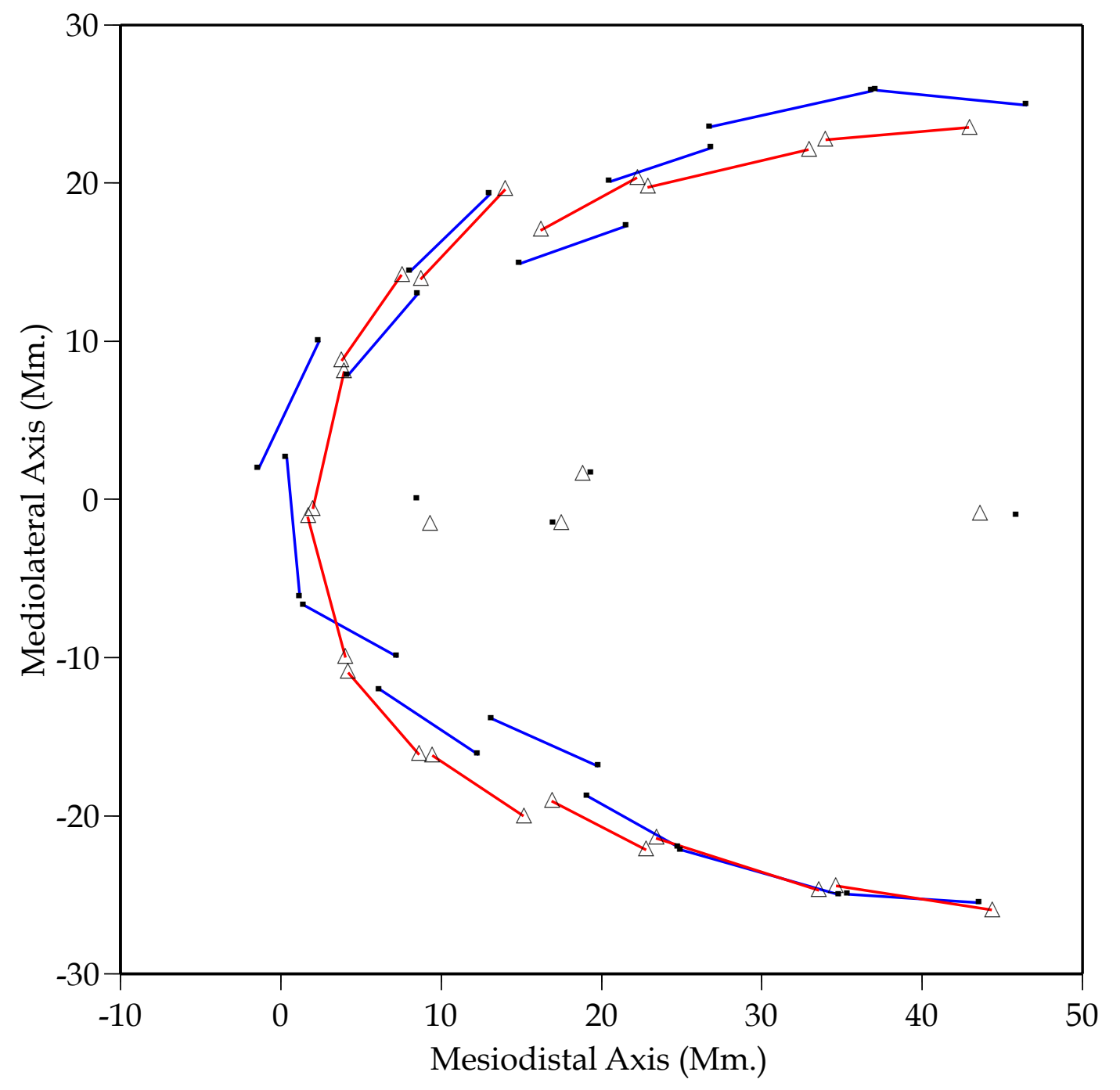

Fig. A-48. Schematic plot of the in-treatment changes in tooth position for case 2012. Registration is on the medial tips of the posterior palatal rugae. Symbols: dots are pretreatment; triangles are posttreatment. 


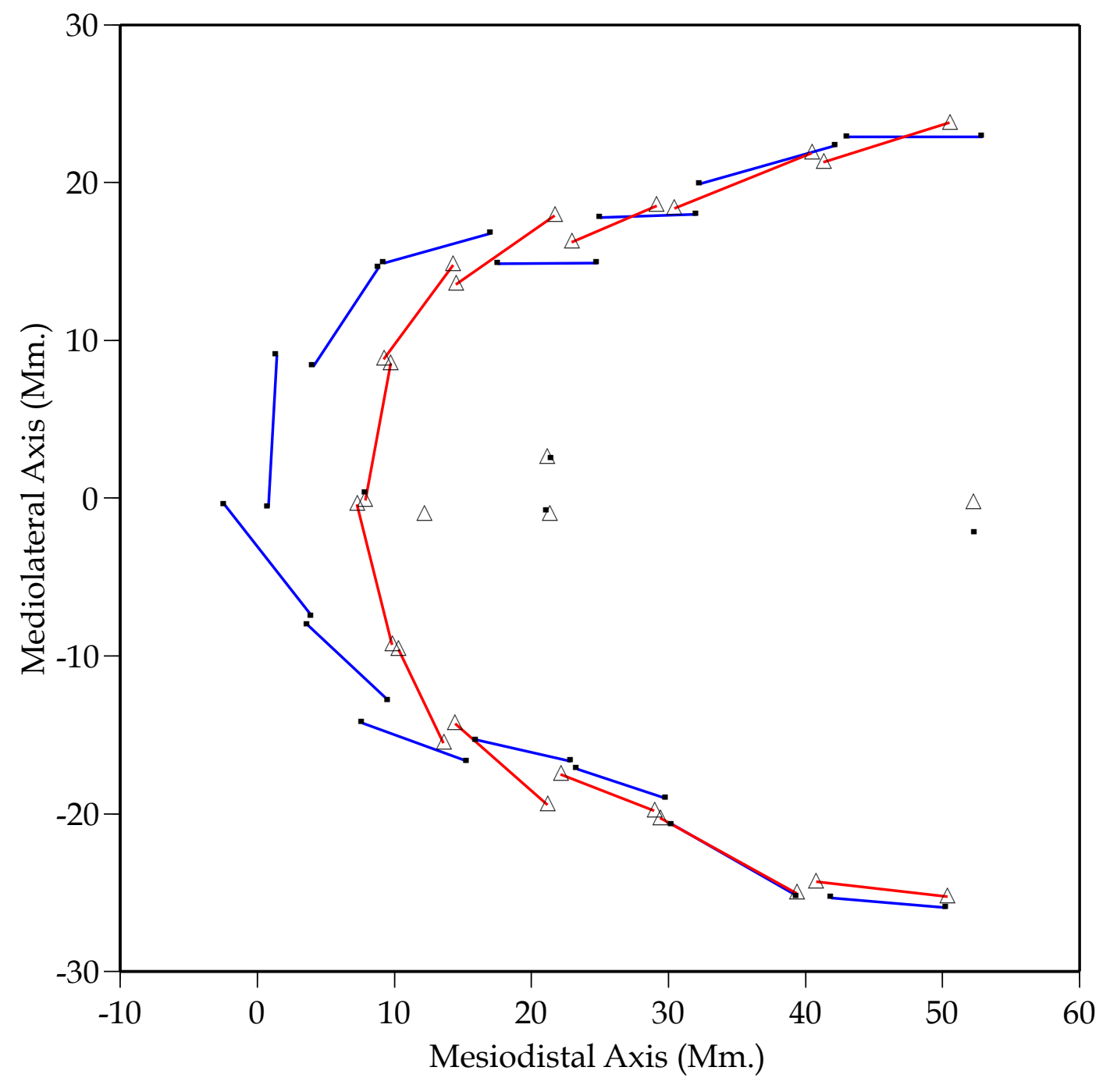

Fig. A-49. Schematic plot of the in-treatment changes in tooth position for case 1352. Registration is on the medial tips of the posterior palatal rugae. Symbols: dots are pretreatment; triangles are posttreatment. 


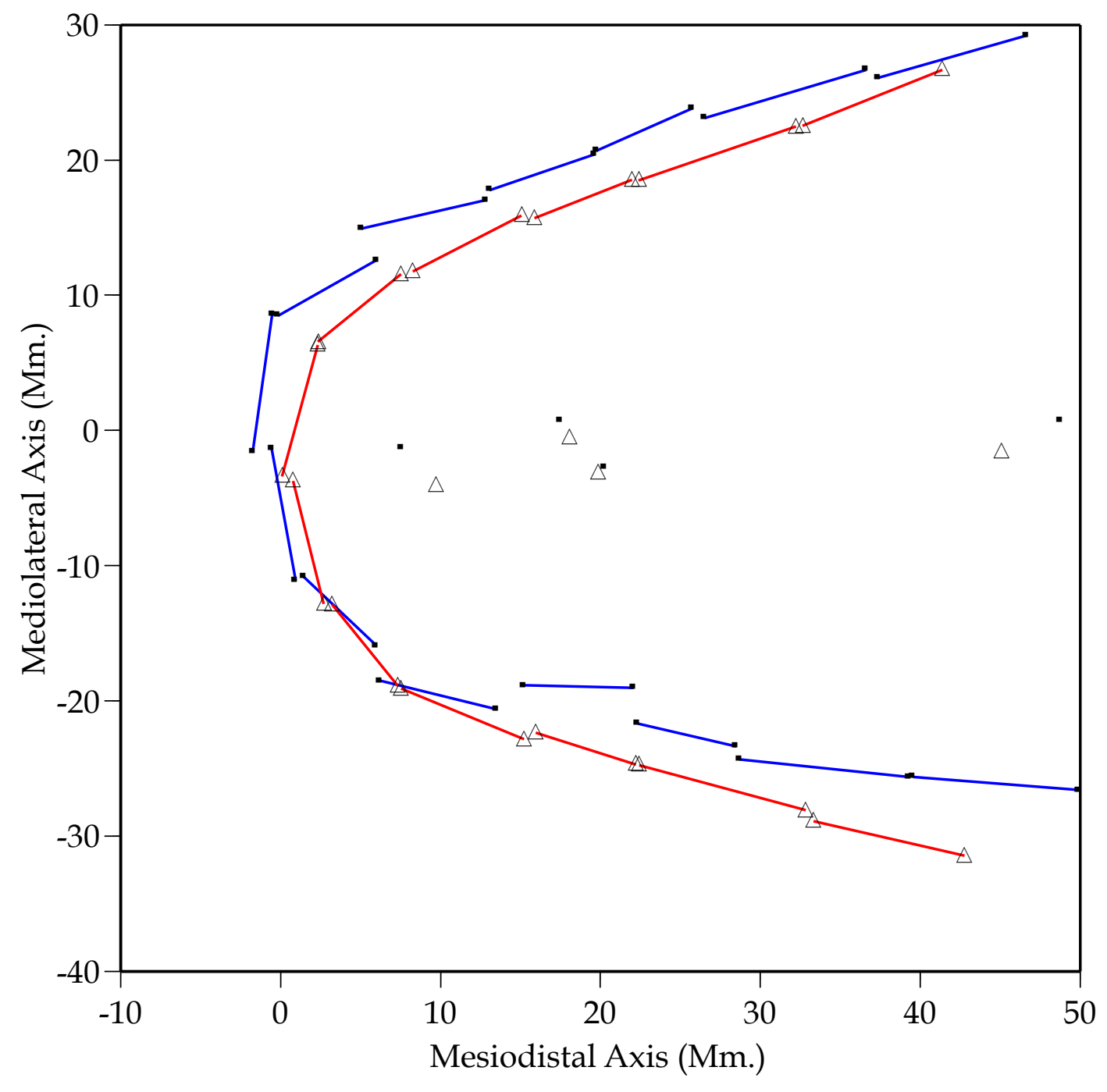

Fig. A-50. Schematic plot of the in-treatment changes in tooth position for case 730. Registration is on the medial tips of the posterior palatal rugae. Symbols: dots are pretreatment; triangles are posttreatment. 
Ryan Gregory Bowles was born in Knoxville, Tennessee, on April 8, 1976. He attended elementary school and graduated from Farragut High School in May, 1994 in Farragut, Tennessee. The following August he entered The University of Tennessee, Knoxille and in August, 1999 received the degree of Bachelor of Science in Predentistry. He entered Covenant Theological Seminary in Saint Louis, Missouri in August, 1997 and received a Graduate Certificate in Biblical and Theological Studies in May, 1998. He entered The University of Tennessee School of Dentistry in Memphis in August 1998 and in June, 2002 received the degree of Doctor of Dental Surgery. In August, 2002 he entered The University of Tennessee, Memphis as a graduated student in Orthodontics and will receive his Master of Dental Science degree in May, 2005.

He plans to join his father in a private practice specializing in orthodontics in Knoxville, Tennessee. Future plans include his practicing internationally in connection with a mission organization. 